\title{
STRUCTURE \\ - OF - \\ BROKEN SPACETIME SYMMETRIES
}


Copyright (C) 2020 by Bernardo Finelli All rights reserved

No part of this publication may be reproduced, stored in a retrieval system, or transmitted in any form or by any means, electronic, mechanical, photocopying, recording or otherwise, without the prior permission in writing from the author.

ISBN:

Printed by: Layout by:

Cover design by:

Original cover photo by:
978-94-6416-060-4

Ridderprint

Bernardo Finelli

Bernardo Finelli

NASA, ESA, the Hubble Heritage Team (STScl/AURA), A. Nota (ESA/STSCl), and the Westerlund 2 Science Team 


\title{
Structure of Broken Spacetime Symmetries
}

\author{
Structuur van Gebroken Ruimtetijd Symmetriëen
}

(met een samenvatting in het Nederlands)

\section{Proefschrift}

ter verkrijging van de graad van doctor aan de

Universiteit Utrecht

op gezag van de

rector magnificus, prof.dr. H.R.B.M. Kummeling,

ingevolge het besluit van het college voor promoties

in het openbaar te verdedigen op

woensdag 26 augustus 2020 des middags te 2.30 uur

door

\section{Bernardo Finelli de Moraes}

geboren op 19 februari 1991

te Sao Paulo, Brazilië 



\section{CONTENTS}

I PRELIMINARIES

I PREFACE 3

1.1 Introduction

1.2 Symmetry breaking and effective theories 4

1.3 Spacetime and gravity 7

1.4 Inflation 8

1.5 Thesis outline 11

2 SYMMETRY AND GROUPS 13

2.1 Spacetime symmetries 13

2.1.1 Physical definition 13

2.1.2 Abstract definition 15

2.2 Groups and their Levi decomposition $\quad 16$ 2.2.1 Supergroups 18

3 THEOREMS 21

3.1 For any symmetry 21

3.1.1 Noether's theorem 21

3.1.2 Global charge conservation 23

3.2 For broken symmetries 25

3.2.1 Goldstone's theorem 25

3.2.2 Fabri-Picasso theorem 26

II TOOLS

4 COSET CONSTRUCTION 31

4.1 Introduction 31

4.2 Homogeneous spaces 32

4.3 Normalization procedure 34

4.4 Maurer-Cartan procedure 38

4.4.1 Goldstone's theorem, revisited 40

4.5 Quasi-invariants 42

4.6 Gauge symmetries 45

4.7 Discussion 47

5 CONSTRUCTION FOR DIFFEOMORPHISMS 49

5.1 Introduction 49 
5.2 Normalization procedure, revisited 50

$5 \cdot 3$ Stückelberg procedure 53

5.4 Example: Effective theory of cosmology 55 5.4.1 In terms of the Stückelberg field 59

5.5 Discussion 62

III CASE STUDIES

6 Shift-SYMMetric COSMOLOgies 65

6.1 Introduction 65

6.2 Broken time translations 67

6.2.1 Non-gravitational systems 67

6.2.2 EFT of cosmology 70

6.3 Constraints on EFT parameters 74

6.3.1 Procedure 74

6.3.2 Relations for $\mathcal{L}\left(\mathrm{g}^{00}\right) \quad 76$

6.3.3 Relations for $\mathcal{L}\left(\mathrm{g}_{00}, \mathrm{~K}_{\mu v}\right) \quad 79$

6.4 Infrared structure 81

6.4.1 Adiabatic modes 81

6.4.2 Soft theorems 84

6.5 Some phenomenology for $\mathcal{L}\left(g^{00}\right) \quad 89$

6.5.1 Ultra slow roll inflation 90

6.5.2 Consistency relation 92

6.5.3 Non-Gaussianities 94

6.5.4 Cutoff scale 97

6.6 Discussion 99

7 GOLDSTONE HiERARCHIES IOI

7.1 Introduction 101

7.2 Coset ordering 103

7.2.1 Levi orderings 104

7.2.2 Example: Curve geometry 106

7.3 Hierarchies in flat spacetimes 110

7.3.1 Vectors: Conformal to Poincaré 110

7.3.2 Tensors: Special galileon 113

7.4 Hierarchies in curved spacetimes 118

7.4.1 Vectors: Poincaré to de Sitter 118

7.5 Discussion 129 
IV CONCLUSION

8 CONCLUSION 133

V APPENDIX

A LIST OF RELEVANT GROUPS 137

A.1 Finite groups 137

A.1.1 Poincaré group 137

A.1.2 Galileo group 138

A.1.3 Carroll group 139

A.1.4 Conformal group 140

A.1.5 de Sitter group 141

A.2 Gauge groups 141

A.2.1 Full diffeomorphism group $\quad \mathrm{I}_{42}$

A.2.2 Cosmology diffeomorphism group $\quad \mathrm{I}_{42}$

B ISOMETRIES OF DE SITTER 145

BIBLIOGRAPHY 153 


\section{LIST OF FIGURES}

Figure 1.1 Left: Some consider Euler's identity the most beautiful equation in math. Right: The anglerfish isn't math, but it's not any less beautiful. (photo by D. Shale [6]) 4

Figure 1.2 The temperature of the CMB is nearly homogeneous. (image by [19]) 9

Figure 1.3 The peaks in the CMB power spectrum have the pattern of acoustic oscillations with adiabatic initial conditions. (image by [19]) 10

Figure 3.1 The boundary of the spacetime manifold $\mathcal{M}$ consists of two spacelike Cauchy surfaces $\Sigma_{2}$ and $\Sigma_{1}$ with a timelike surface $S$ located at infinity enclosing the two. 24

Figure 7.1 Leo's notion of curve drawing is to slide a ruler without slipping around a fixed circle, pushing the pen with the ruler. Here, Leo has kept the pen still with respect to the ruler. We draw a full rotation cycle, though we have included only five positions of the ruler to simplify the image. $\quad 108$

\section{CONVENTIONS}

- Spacetime metrics have mostly plus signature, i.e., $(-+++$ ...).

- We denote by $\mathrm{D}$ a spacetime's number of dimensions. The number of space dimensions is $d=D-1$.

- Indices $\mu, v$ are curved spacetime indices ranging from 0 to $d$. 
- Indices $\alpha, \beta$ are flat spacetime ranging from 0 to $d$.

- Indices $i, j$ are space indices ranging from 1 to $d$.

- Indices $a, b$ are internal.

- Trivial de Rham cohomology is always assumed, meaning all closed differential forms are exact, $\mathrm{d} \alpha=0 \leftrightarrow \alpha=\mathrm{d} \beta$.

- By "group" we always mean a Lie group, unless explicitly said otherwise. By the same token, a "finite group" means a Lie group of finite dimension, not a group with a finite number of elements.

- By default, transformations are given in passive form (coordinates can transform). When they are given in active form (coordinates don't transform), we will say so explicitly.

- Any content in a shaded box such as this one is an example that supplements the text. The reader already familiar with the concepts explained in the text can freely skip these. 



\section{PUBLICATIONS}

Ideas and figures of this thesis have appeared previously in the following publications:

- Bernardo Finelli. "Inequivalent Goldstone hierarchies for spontaneously broken spacetime symmetries." In: Journal of High Energy Physics 2020.75 (March 2020). arXiv: 1909.11077, p. 25. ISSN: 1475-7516. DOI: https:/ / doi.org/10.1007/JHEPo3(2020)075. URL: https://arxiv.org/abs/1909.11077

- Section 4.3 of Chapter 4 , Chapter 5 and Chapter 7 are based on this article.

- Bernardo Finelli, Garrett Goon, Enrico Pajer and Luca Santoni. "The Effective Theory of Shift-Symmetric Cosmologies." In: Journal of Cosmology and Astroparticle Physics 2018.05 (May 2018). arXiv: 1802.01580, p. 060. ISSN: 1475-7516. DOI: 10.1088/14757516/2018/o5/o6o. uRL: https://arxiv. org/abs/1802.01580

- Chapter 6 is based on this article.

- Bernardo Finelli, Garrett Goon, Enrico Pajer and Luca Santoni. "Soft Theorems for Shift-Symmetric Cosmologies." In: Physical Review D 97.06 (March 2018). arXiv: 1711.03737, p. 3531. ISSN: 2470-0029. DOI: 10.1103/physrevd.97.063531. URL: https:// arxiv.org/abs/1711.03737

- Chapter 6, predominantly Section 6.4, is based on this article. 

Part I

PRELIMINARIES 



\section{I.I INTRODUCTION}

Mathematics is famously successful at describing the physical laws that govern nature. This is somewhat surprising, for not everything in the universe can be encoded in math-love, art and the reproductive behavior of the anglerfish being some of many examples. It seems almost random that physics can.

Much has been written on the subject, but one of the reasons at the heart of this is symmetry, the idea two things that look nothing alike are actually intrinsically linked to each other. Physical symmetries link different states of the universe, and as such the universe's evolution must preserve this link. In other words, symmetries render physical laws structured. And mathematics is nothing but the study of structure.

It's thus no wonder that the more symmetrical a system is, the simpler its study is. Indeed, all fundamental theories we have discovered so far-general relativity and the Standard Model of particle physics-are inexorably built from the underlying symmetries of physics.

And yet, symmetry renders things incredibly boring. The laws of physics treat all moments of time and locations in space the same- therefore the universe should look the same everywhere at all times? No, of course not. That would be a rather uninteresting universe! We need symmetries to make physics orderly, but too much order prevents any kind of complexity.

Spontaneous symmetry breaking is how nature gets to eat its cake and have it too: it keeps the order and simplicity of the laws of physics, while allowing our system to dynamically evolve from a symmetric configuration to an asymmetric one. It's an essential feature of our universe, connecting a myriad of disparate phenomenon such as the formation of ice crystals [1], the mass 
Figure 1.1: Left: Some consider Euler's identity the most beautiful equation in math. Right: The anglerfish isn't math, but it's not any less beautiful. (photo by D. Shale [6])
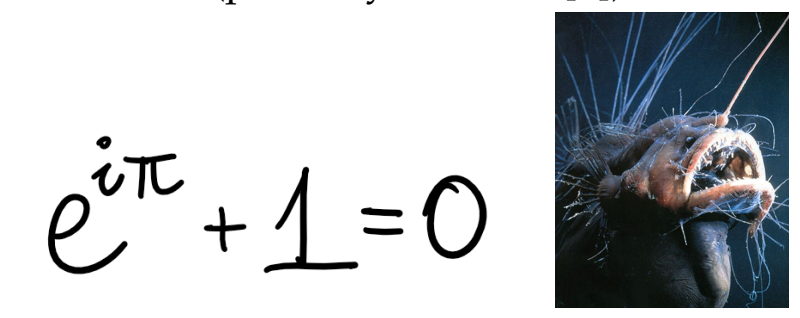

of electrons $[2,3]$, the expansion of the universe [4], and cancer metastasis [5].

\subsection{SYMMETRY BREAKING AND EFFECTIVE THEORIES}

The phenomenon of spontaneous symmetry breaking in physics occurs whenever the dynamic laws of a system display invariance under a certain transformation, yet the system's ground state (state of lowest energy) does not. Classically, this means the action $S$ is invariant, but the ground state solution to our fields $\bar{\psi}$ isn't:

$$
\delta S=0 \text { but } \delta \bar{\psi} \neq 0,
$$

where $\delta$ denotes the infinitesimal variation. The statement is similar quantum mechanically. Instead of a background solution $\bar{\psi}$, we have a vacuum state $|0\rangle$, which can be acted upon by the symmetry generator $\mathrm{Q}$. Then:

$$
\delta z=0 \text { but } \mathrm{Q}|0\rangle \neq 0,
$$

where $z=\int \mathcal{D} \psi e^{i \psi}$ is the path integral. Of course, not all symmetries have to be broken; typically there will still be some left for which $\delta \bar{\psi}=0$.

In view of this, broken symmetry is somewhat of a misnomer. The laws of physics are still invariant, just the vacuum that isn't. At the perturbative level-where the system is described as fluctuations on top of the background-the broken symmetry manifests 
itself in a deeper, less obvious manner. We will discuss this later. Right now, the most important thing to remember is that, if the perturbative degrees of freedom are $\varphi=\psi-\bar{\psi}$, then $\varphi$ behaves differently under the symmetry depending on whether $\bar{\psi}=0$ or $\bar{\psi} \neq 0$. Breaking the symmetry changes the nature of these degrees of freedom, that is to say, they describe different objects. Furthermore, even if all old objects were massive, some of these new objects might be massless. They are called Nambu-Goldstone particles, or just Goldstones for short. Under usual circumstances, there is one massless Goldstone for each broken symmetry.

Now, this is all very well and good, but the reason why spontaneous symmetry breaking is of such importance in physics is due to its role as an organizational principle for effective field theories (EFTs). The nature of the degrees of freedom that describe a system's dynamics never remain unscathed under renormalization flow, that is to say, the very particles of the field theory itself change at different scales.

The basic example is quantum chromodynamics (QCD). In the deep UV, the theory is perturbative and we can happily work with the fundamental quarks. As we move towards the IR, the coupling becomes stronger and stronger until it eventually blows up-the famous Landau pole. Obviously, this doesn't mean physics literally ceases to exist at that point, merely that the theory is no longer perturbative. At the same time, this can be taken to suggest that, for most intents and purposes, it flat out doesn't make sense to talk about quarks at length scales larger than a nucleon.

Indeed, the "good" degrees of freedom at nuclear scales aren't really quarks, but hadrons. And if we build our field theory with these good hadrons, then the theory is perturbative as well! Of course, this theory is merely an effective theory, not a fundamental one. Quantities such as the masses of pions can't be calculated but must be measured. To compute them, we would need to solve nonperturbative QCD.

Breaking a symmetry reshuffles the degrees of freedom in a theory. So too does flowing over a Landau pole. Are the two related? Yes, of course. Perturbativity restoration in a field theory is typically accompanied by symmetry breaking. At short lengths or high 
temperatures, the symmetry is unbroken and we work with the more fundamental degrees of freedom. At long lengths or low temperatures, the symmetry is broken and we shift to the effective ones. Some examples:

- Quarks into hadrons. This is the chiral symmetry breaking, with pions ${ }^{1}$ as Goldstones. [7]

- Molecular description of fluids into continuum fluid dynamics. This involves breaking Lorentz symmetry, and the Goldstones are phonons. [8]

- The splitting of the electroweak interaction into electromagnetism and weak force. This is the electroweak breaking or Higgs mechanism; there are no Goldstone in this case. $[2,3]$

The pattern is then a repetitive one: more fundamental theories morph into effective ones at the moment they lose perturbativity, or unitarity of the S-matrix. Unitarity is restored by rearranging the degrees of freedom as dictated by the associated symmetry breaking pattern. Conversely, each EFT has a maximum energy scale called a cutoff before they lose unitarity, at which point the symmetry is restored and the more fundamental theory must be reintroduced as a UV completion. To study symmetry breaking is then of paramount importance in physics, as it sheds light on how all theories organize themselves at all energy scales.

One uses the language of mathematics to discuss symmetries, and broken symmetries are no exception. Group theory, in particular Lie group theory, is what governs all this discussion. The machinery for such was first employed for physics in 1969 [9, 10]. Indeed, with only knowledge of the symmetry breaking pattern, one can build the most general action that displays a symmetry in the "less obvious manner" we discussed before, because there aren't that many possibilities to realize symmetries in these special ways. Thus, with a single general EFT, we can write down every possible model capable of describing the system. This is very well understood for internal symmetries, but physics display another

I Strictly not massless, as the chiral symmetry is merely approximate, but good enough for our purposes here. 
kind of symmetry whose breaking is the central theme of this thesis: spacetime symmetries.

\subsection{SPACETIME AND GRAVITY}

For most of the history of physics, space and time were seen as just the stage where the actors of physics performed their play. On occasion, things were shaken up a bit-first in the 19th century when Bernhard Riemann showed space needn't be flat, then in the early 2oth century when Hendrik Lorentz and Albert Einstein fused the two into just spacetime-but the essential paradigm was unaltered: spacetime is merely an ambient, an environment.

Then all changed about 10o years ago, when Einstein linked spacetime and matter through the phenomenon of gravity:

$$
\mathrm{G}_{\mu \nu}+\Lambda g_{\mu \nu}=\frac{1}{2 \mathrm{M}_{\mathrm{Pl}}^{2}} \mathrm{~T}_{\mu \nu},
$$

where $G_{\mu \nu}$ and $g_{\mu \nu}$ encode information about the geometry of the curved spacetime, while $T_{\mu \nu}$ is the stress-energy tensor containing information about the matter within the spacetime. The constants $\mathrm{M}_{\mathrm{Pl}} \sim 10^{18} \mathrm{GeV}$ and $\Lambda \sim 10^{-66} \mathrm{eV}^{2}$ (strictly nonzero) are the free constants of general relativity.

The Einstein field equations encode the dynamics of spacetime: it's no longer just an ambient supplied for the physical objects of a theory to live in, but a physical object in and of itself. It evolves and changes as determined by the matter within. We call the coupling of the two gravity.

The EFTs of particle physics are governed by the breaking of internal symmetries. As for gravitational systems, the connection between spacetime and gravity then logically establishes their relation with the breaking of spacetime symmetries. Several gravitational systems can be understood under this lens, allowing us to construct model-independent EFTs for them. Examples include:

1. The Big Bang breaks time translation symmetry. Cosmological perturbations can be seen as the degrees of freedom of the associated EFT. [4] 
2. A black hole breaks spatial translation (and rotations, if it has angular momentum). Quasinormal modes are then the degree of freedom of this EFT, being of immense importance for gravitational wave astronomy. [11]

3. String theory is a gravitational theory living in a higher dimensional spacetime. String-inspired EFTs of gravitational systems are often governed by the breaking of symmetries of the higher dimensional space. [12]

The interplay between broken internal symmetries and EFTs is well-understood, but for broken spacetime ones, research is still ongoing. This is because many of the theorems establishing the basic properties of such EFTs find their core assumptions violated when spacetime symmetries are broken. Perhaps the most infamous example is the disappearance of Goldstone bosons, that is, we no longer have one boson for each broken symmetry. We call the missing bosons inessential [13-15].

Much work has been done in the past years to understand the structure of broken spacetime symmetries as well as the properties and uniqueness of the associated EFTs, specially when some bosons are seemingly inessential. We will look this issue more deeply in Chapter 7 .

\subsection{INFLATION}

If we look at the night sky, the universe appears to be homogeneous. The large scale structure (LSS) of matter stars becoming uniform at around 10 Mpc [16], and the largest known structures, galactic filaments, end at around 10o Mpc [17]. Meanwhile, the temperature of the cosmic microwave background (CMB) is the same, up to one part in $10^{5}$, no matter in which direction we look [18].

Yet this homogeneity isn't perfect. Small fluctuations exist on top of this uniform background. Several probes, the most recent being Planck, have measured the power spectrum (i.e., distribution of perturbations in momentum space) of the CMB fluctuations. Furthermore, these fluctuations are small and, for most of the universe's history, everything in it is described by two theories we 


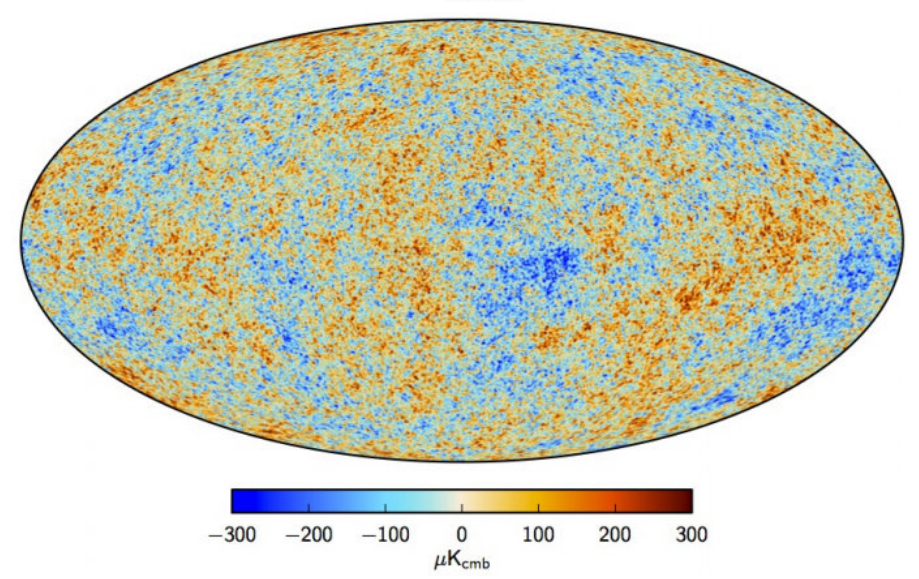

Figure 1.2: The temperature of the CMB is nearly homogeneous. (image by [19])

know well, general relativity and the Standard Model, plus some dark matter and dark energy ${ }^{2}$. This means we can simply evolve the CMB backwards in time, as far back as we can rely on these theories. This outputs the primordial power spectrum, the ultimate seed of all cosmological fluctuations, from hot spots in the CMB to galaxy clusters.

The CMB and primordial power spectra reveal two crucial features:

- The CMB power spectrum has the adiabatic pattern for its acoustic peaks (see Figure 1.3). These peaks deal with the gravitational interplay between the radiation-baryon fluid (coupled via Compton scattering) and the pressureless dark matter fluid [20]. If the peaks are adiabatic, then the fluctuations of photons, baryons and dark matter don't constitute independent random variables; rather, they all have the same common source.

2 We might not known what they are, but for large scale cosmology it's sufficient to treat the former as just a pressureless fluid and the latter as a cosmological constant. 


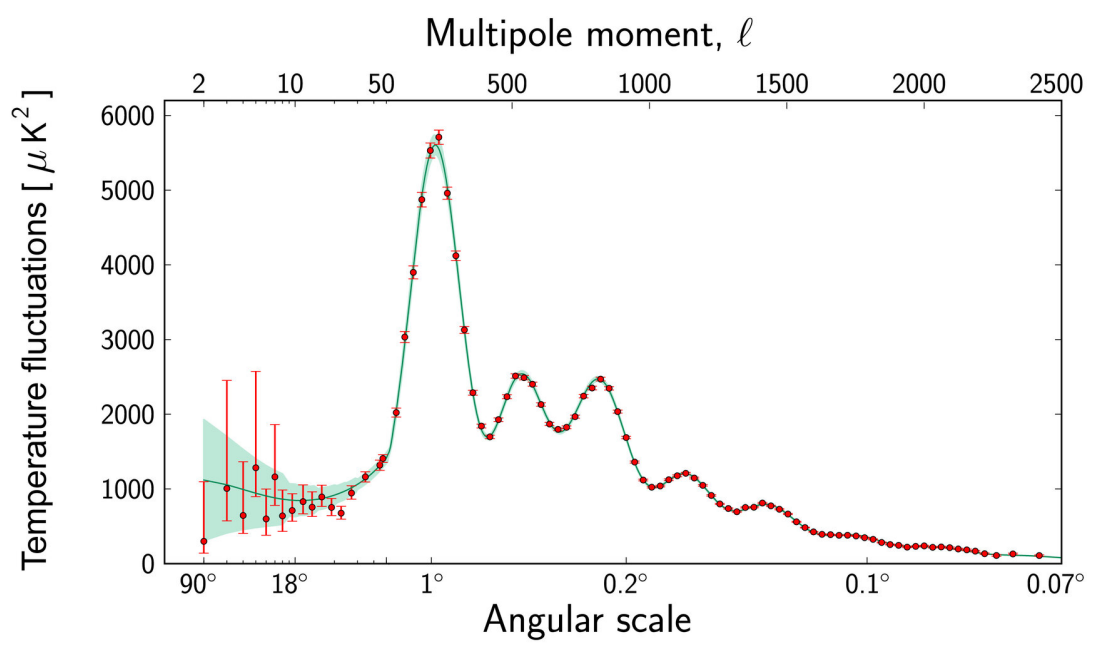

Figure 1.3: The peaks in the CMB power spectrum have the pattern of acoustic oscillations with adiabatic initial conditions. (image by [19])

- The primordial power spectrum $P(k)$ is $P(k) \sim k^{3}$, which implies the correlations of primordial fluctuations are nearly scale invariant.

Now, we could simply declare that primordial fluctuations are produced during the quantum gravitational era of the universe that we can't hope to explain in the present, and that's it, the story ends. However, if we wish to be a bit more ambitious, we can try to explain them by a dynamical process below the Planck scale, without invoking the nonperturbative quantum gravity black box.

One such process is inflation, a period in the past when the universe underwent accelerated expansion. To explain adiabaticity (single source of perturbations), inflation is typically constructed in terms of a single scalar field, the inflaton- whether it's composite or elementary doesn't matter. And to explain near scale invariance, this field should source a period of quasi-de Sitter acceleration, as rescalings of space are an isometry of de Sitter (see Appendix B).

Inflation's success at explaining these two properties have resulted in its wide acceptance in cosmology [21]. As we don't know the fundamental theory of inflation, an effective theory approach, 
built out of the breaking of time translations [4], is essential lest we get drowned in an infinitude of putative inflationary models. Furthermore, concerns arise when trying to embed inflation in a more fundamental theory, typically string theory, and additional broken symmetries are often invoked to tame the issues. We will see this in more detail in Chapter 6.

\subsection{THESIS OUTLINE}

This thesis is structured as follows:

- Part i continues our presentation of preliminary and background material relevant to Lie group theory, symmetry breaking, and effective field theories.

- In Chapter 2, we define the central concept of this thesis, spacetime symmetry groups, and discuss aspects of group theory we will use, in particular the Levi decomposition.

- In Chapter 3, we introduce the theorems that form the basis of symmetry and symmetry breaking in physics: Noether's theorem leading to local (and sometimes global) charge conservation, Goldstone's theorem dealing with the nature of Goldstone bosons, and the Fabri-Picasso theorem indicating the existence of superselection sectors when symmetries are broken.

- Part ii introduces tools, methodologies and algorithms for building effective field theories out of a symmetry breaking pattern.

- In Chapter 4, we present the coset construction, which deals with global groups or global groups that have been gauged. We introduce a novel way to perform this construction via normalization of invariants and relate it to the usual one in the literature via the Maurer-Cartan form.

- In Chapter 5, we discuss diffeomorphism groups which are a different kind of gauge group. We outline how to 
employ the normalization procedure in this case, and do so for the example of cosmology.

- Part iii applies the tools of the previous part in specific and extended examples.

- In Chapter 6, we employ the tools of broken diffeomorphisms and broken internal symmetries to construct the effective field theory of shift-symmetry cosmology.

- In Chapter 7, we discuss the Goldstone hierarchies displayed by certain theories, and carefully analyze various theories in flat and curved spacetimes.

- Part iv closes this thesis with a synthesis of this work and discussion of future prospects.

- Part $\mathrm{v}$, the appendix, collects supplementary material referred through the main body of this work.

- In Appendix A, we list all groups used in this work. This includes global groups, with their algebra and matrix representation of the algebra, as well as their Levi decompositions. Relevant diffeomorphism groups are also listed.

- In Appendix B, we give the explicit expression for the finite isometries of de Sitter space. This is used in Chapter 7 , but would take too much space there. 
SYMMETRY AND GROUPS

\subsection{SPACETIME SYMMETRIES}

Since spacetime symmetries are the central concept of this thesis, it might be worth investigating what they even are to begin with.

\subsubsection{Physical definition}

If we are willing to refer to a physical spacetime and its isometry group, then a natural classification of symmetries follows.

First, we have symmetries under which the spacetime coordinates themselves transform, $\delta x \neq 0$. Moreover, we demand spacetime be homogeneous and symmetric, which means these symmetries include some notion of time shift, space shift, boosts mixing time and space and rotations mixing space. We require this as otherwise the symmetry group lacks sufficient structure to construct a usable effective field from a symmetry breaking pattern.

Homogeneous and isotropic symmetries are also called kinematic and the complete classification of kinematic algebras is known $[22,23]$. However, for physics, kinematic symmetries must be compatible with a metric structure, that is to say, they must contain the isometries of some spacetime metric. For instance, the group $\mathrm{SO}(2,4)$ contains the Poincare $\mathrm{ISO}(1,4)$ which is the isometry of Minkowski space: this is valid. On the other hand, if the group isn't compatible with a metric, then it's not immediately clear if it's of any use in describing gravitational systems, so they aren't of interest to us in this work.

The kinematic generators, together with all others with whom they nontrivially commute, are spacetime symmetries. The latter type of generator includes those of nonuniform symmetries. These are transformations purely of the fields that are spacetimedependent, i. e., $\delta \psi=f(\psi ; x)$. It can also include closure generators 
that transform the fields uniformly, $\delta \psi=f(\psi)$, and so naively don't look like spacetime symmetries, but still have to be added to the bunch for group closure.

Meanwhile, a generator that doesn't fall into the description above is internal.

To distinguish these categories, compare these two examples and in particular how the nature of the $Q$ generator changes.

EXAMPLE: STANDARD SHIFTS. Consider this algebra containing translations $\mathrm{P}$, Lorentz generators $\mathrm{M}$, together with a scalar $\mathrm{Q}$. Besides the usual Poincaré algebra, we have:

$$
[\mathrm{P}, \mathrm{Q}]=0 .
$$

An action for a single field displaying this symmetry is:

$$
S=\int d^{4} x P(X),
$$

for $X=(\partial \phi)^{2} / 2$, where $\phi$ is some scalar field and $P(X)$ a free function. The symmetry transformations are:

$$
x \rightarrow \Lambda x+a \quad \phi \rightarrow \phi+c,
$$

where $\Lambda$ is a Lorentz matrix, $a$ a translation vector and $c$ the shift induced by $\mathrm{Q}$.

There's no deep mystery here. Obviously the Poincaré generators are kinematical, and correspond directly to the isometries of the underlying flat spacetime metric. Meanwhile, Q commutes with everything; it's merely a simple field shift.

EXAMPLE: Galileon Shifts. Now take the shift algebra from the previous example and enlarge it by adding a vector generator of the form $B_{\mu}$. Since it's a vector, its commutation with $M_{\mu \nu}$ is given by its tensorial structure. The nontrivial commutators are given by:

$$
\left[\mathrm{P}_{\mu}, \mathrm{B}_{\nu}\right]=\eta_{\mu \nu} \mathrm{Q} \quad\left[\mathrm{P}_{\mu}, \mathrm{Q}\right]=0 \quad\left[\mathrm{Q}, \mathrm{B}_{\mu}\right]=0 .
$$


An example (not the most general one) of an action with this symmetry is:

$$
S=\int d^{4} x\left[\frac{1}{2}(\partial \phi)^{2}+(\square \phi)(\partial \phi)^{2}\right],
$$

with the group transformation law:

$$
x \rightarrow \Lambda x+a \quad \phi \rightarrow \phi+c+b \cdot x,
$$

where $b_{\mu}$ is a vector serving as the parameter of $B^{\mu}$.

Now the situation is more interesting. Of course, $P_{\mu}$ and $M_{\mu v}$ are still kinematical generators. But $B_{\mu}$ doesn't commute with either, so now it constitutes a nonuniform symmetry. Indeed, we can see from how it effects a spacetime-dependent shift on the field $\phi$, i. e., $\mathrm{B}^{\mu} \phi=\chi^{\mu}$. What about the Q? It still looks like the same uniform shift as before, $\mathrm{Q} \phi=1$, and it commutes with everything. Yet it's required for closure of the algebra of the $P_{\mu}$ and $B_{\mu}$, which are spacetime generators. So $Q$ is spacetime, too.

\subsubsection{Abstract definition}

Alternatively, we can define spacetime and internal symmetries in a purely abstract manner, without reference to an actual spacetime manifold. This is perhaps more economical, but less physically transparent. Any Lie group can be decomposed into compact and noncompact pieces:

$$
\mathrm{G}=\mathrm{S} \times \mathrm{I},
$$

where $S$ is noncompact, I is compact. We don't further specify the structure of $S$ and I-they can be simple or semisimple or not simple at all. The elements of $S$ are then spacetime symmetries while those of I are internal.

To understand why this definition has anything to do with the previous one, recall what we demand that some of the spacetime symmetries be kinematic. This means some notion of boosts exist. 
Furthermore, we require the kinematic symmetries be compatible to a metric with signature $(-+++\ldots)$. The boosts mix time and space together, which have different signatures-their group is noncompact. ${ }^{1}$ All other spacetime symmetry generators don't commute with boosts, or don't commute with generators that don't commute with boosts, and so on, so they all collect in the noncompact factor $S$; none go in I.

Meanwhile, internal symmetries must be compact in order for the Hamiltonian to be bounded from below. So they all go in I and not $S$.

Of course, this abstract definition will not work for a spacetime that is not maximally symmetric. For example, the isometry group of the Schwarzschild metric is $\mathrm{SO}(3)$, which is compact. It will also not work with diffeomorphism groups, as such groups aren't even abstract to begin with, which we discuss in Section 5.1.

\subsection{GROUPS AND THEIR LEVI DECOMPOSITION}

Symmetries are naturally described in the language of Lie groups. What we're really interested in in this section is the classification of groups, but first let's quickly refresh what a Lie group is.

A Lie group consists of a differential manifold equipped with a product for the objects within the manifold. By axiom, the product must be smooth, associative (no need for parentheses), closed (the output lives on the manifold), and invertible. A symmetry transformation is what we call a group action: a realization of this group on any kind of object while preserving these axioms.

Smoothness and invertibility imply the Lie group contains an identity element for its product. Much in the same way we can approximate a Riemann manifold around a point by a vector space (the tangent plane), we can approximate the group manifold around the identity by a vector space too: the Lie algebra.

1 If the reader prefers a brute force argument: homogeneity, isotropy and metric structure imply a maximally symmetric metric, so either Minkowski, de Sitter or anti-de Sitter. The three isometry groups are $\operatorname{ISO}(1, \mathrm{~d}), \mathrm{SO}(1, \mathrm{D})$ and $\mathrm{SO}(2, \mathrm{~d})$, respectively. The three are noncompact. 
With that out of the way, let's talk about how to classify Lie groups. The classification of simple Lie groups is famous and well understood. That of nonsimple ones is not simple, however. A powerful tool for this task is the Levi decomposition which states that any finite Lie group $\mathrm{G}$ can be decomposed as:

$$
\mathrm{G}=\mathrm{R} \rtimes \mathrm{L},
$$

where L is a (semi)simple group called the simple factor (or Levi factor) and R a nonsimple but "nice" Lie group called the radical. Both $R$ and $L$ are unique. ${ }^{2}$ The niceness of the radical is due to its three defining properties:

1. It's an ideal, meaning its algebra $\mathfrak{r}$ is invariant under the algebra $\mathfrak{g}$ of the group:

$$
[\mathfrak{r}, \mathfrak{g}] \subseteq \mathfrak{r} .
$$

2. It's solvable, meaning the algebra telescopes to zero upon repeated commutations:

$$
\begin{gathered}
{[\mathfrak{r}, \mathfrak{r}]=\mathfrak{r}_{1} \subset \mathfrak{r}} \\
{\left[\mathfrak{r}_{1}, \mathfrak{r}_{1}\right]=\mathfrak{r}_{2} \subset \mathfrak{r}_{1},} \\
{\left[\mathfrak{r}_{2}, \mathfrak{r}_{2}\right]=\mathfrak{r}_{3} \subset \mathfrak{r}_{2},} \\
\vdots \\
{\left[\mathfrak{r}_{\mathfrak{n},}, \mathfrak{r}_{\mathrm{n}}\right]=0,}
\end{gathered}
$$

for finite $n$.

3. It's maximal, meaning it's the largest subalgebra $\mathfrak{r} \subseteq \mathfrak{g}$ with the above properties.

Radicals admit no complete classification the way simple groups do. Through this work, if we encounter a radical that we don't

2 Strictly speaking, the decomposition is unique for the Lie algebra, not the group, as the latter can have additional discrete group factors encoding topology. Furthermore, the simple factor is only unique up to adjoint conjugation by the group's nilradical. These are technicalities of little import for our purposes here. 
believe is immediately recognizable as a familiar group, we will simply denote it by the exponential of the span of its generators $S$, i. e., $R=\exp \langle S\rangle$.

The quintessential Levi decomposition in physics is the one for the Poincaré group $\operatorname{ISO}(1, \mathrm{~d})$ :

$$
\operatorname{ISO}(1, d)=\mathbb{R}^{\mathrm{D}} \rtimes \mathrm{SO}(1, \mathrm{~d}) .
$$

Extensions of the Poincare group are governed by this decomposition through O'Raifeartaigh's theorem [24]. Any enlargement of the Poincaré group must necessarily meet one of the following three conditions:

1. Trivially enlarge the simple factor: $\mathrm{SO}(1, \mathrm{~d}) \rightarrow \mathrm{SO}(1, \mathrm{~d}) \times \mathrm{I}$ for an internal group I. (Coleman-Mandula theorem)

2. Enlarge the radical: $\mathbb{R}^{\mathrm{D}} \rightarrow R$, for a larger radical $R$.

3. Destroy the decomposition: $\mathrm{ISO}(1, \mathrm{~d}) \rightarrow \mathrm{L}$, for $\mathrm{L}$ a larger simple group.

\subsubsection{Supergroups}

We won't study supersymmetry in this work, but let us briefly outline how the Levi decomposition applies to it. This is important if one wishes to apply the techniques discussed here for broken supergroups displaying Goldstone fermions.

Typically, supersymmetries are presented in terms of a graded Lie algebra, e. g., with commutators and anticommutators. So it's not clear if a superalgebra exponentiates to a supergroup that can be Levi decomposed. Yet, supergroups are still formally defined as just any group: a manifold with a morphism satisfying certain axioms; the only difference is that the manifold can have both real/complex (bosonic) coordinates and Grassmannian (fermionic) coordinates [25]. As such, any superalgebra can be represented as a standard Lie algebra of commutators. The procedure for this is described in $[26,27]$. Then, one can simply Levi decompose the group. 
For example, in the language of superfields, all quantities are functions of the bosonic coordinates $x^{\mu}$ and the fermionic coordinates $\theta^{\alpha}, \bar{\theta}^{\dot{\beta}}$. For the super-Poincaré group, much in the same way that $P_{\mu}$ generates translations of $x^{\mu}$, the supercharges $Q_{\alpha}, \bar{Q}_{\dot{\beta}}$ generate supertranslations of $\theta^{\alpha}, \bar{\theta}^{\dot{\beta}}$ [28]. Applying the Levi decomposition theorem, one then sees that the super-Poincaré group can be written as:

$$
\operatorname{SISO}(1, d)=\exp \langle\mathrm{P}, \mathrm{Q}, \overline{\mathrm{Q}}\rangle \rtimes \mathrm{SO}(1, \mathrm{~d}) .
$$

We notice that supersymmetries are contained within option 2 of O'Raifeartaigh's theorem: they enlarge the radical of the Poincaré group [28]. 

This chapter concerns itself with the basic theorems that govern the structure of physical symmetries:

- For any symmetry, broken and unbroken

- Noether's theorem, which relate a locally conserved current to each symmetry.

- Global charge conservation, which integrate the locally conserved current into a global charge.

- For broken symmetries

- Goldstone's theorem, which relate degenerate vacua under the broken symmetry to massless bosons.

- The Fabri-Picasso theorem, which stipulates the degenerate vacua live in different sectors of the S-matrix.

These theorems are presented for internal symmetries, as they were traditionally first formulated. Then, for each one, we discuss whether or not they apply to spacetime symmetries.

\subsection{FOR ANY SYMMETRY}

\subsubsection{Noether's theorem}

Among all theorems related to symmetry we will recall in this chapter, Noether's (first) theorem is the most important one [29]. It famously describes the local conservation of charge. Proofs of Noether's theorem are legion; in what follows is a simple one. 
We assume active language where only fields transform, so $\delta \psi=$ $\epsilon f(\psi ; x)$ infinitesimally under the symmetry, where $\epsilon$ is constant. Under an arbitrary $\epsilon(\psi ; x)$, the action changes as:

$$
\delta S=\int \epsilon \mathrm{K}+\mathrm{d} \epsilon \wedge \mathrm{J},
$$

where $\mathrm{K}$ is a D-form and $\mathrm{J}$ a $(\mathrm{D}-1)$-form. If the above is a symmetry transformation, the $\epsilon$ is constant (by definition) and the variation of the action must equal a total derivative $\int \mathrm{d}(\epsilon \mathcal{\Lambda})$. Hence:

$$
\mathrm{K}=\mathrm{d} \wedge .
$$

Meanwhile, if the equations of motion are satisfied, then the variation of the action is zero for any $\epsilon$ (by definition), which means:

$$
\epsilon[\mathrm{K}-\mathrm{dJ}]=0 .
$$

If the transformation is a symmetry transformation performed while the equations of motion are satisfied, the two conclusions hold together, and we derive:

$$
\begin{aligned}
\mathrm{d} j & =0 \\
j & =J-\Lambda .
\end{aligned}
$$

The $j$ is therefore the locally conserved current. In practice, to compute it, one writes down the variation (3.1) in terms of the Lagrangian $\mathcal{L}(\psi)$. If the Lagrangian contains higher derivatives, integrations by parts are necessary to put the variation in the form of (3.1). The result is:

$$
\varepsilon j^{\mu}=\left[\frac{\partial \mathcal{L}}{\partial \nabla_{\mu} \psi}-\frac{\partial^{2} \mathcal{L}}{\partial \nabla_{\mu} \nabla_{\nu} \psi} \nabla_{v}+\ldots\right] \delta \psi-\Lambda^{\mu},
$$

where the structure function $\wedge^{\mu}$ must be determined manually by solving $\delta \mathcal{L}=\nabla_{\mu} \wedge^{\mu}$. 
SPACETime symmetries. None of the assumptions behind Noether's theorem make any mention as to whether the symmetry is internal or spacetime. Its conclusion applies to spacetime symmetries unadulterated.

\subsubsection{Global charge conservation}

By Noether's theorem, each internal symmetry of the action leads to a current 3 -form $\mathrm{J}$ that is conserved, $\mathrm{dJ}=0$. The corresponding charge is by definition the integral of this current over some Cauchy surface $\Sigma$ whose normal is future-pointing:

$$
\mathrm{Q}=\int_{\Sigma} \mathrm{J}
$$

Let us now integrate dJ. This is a 4 -form, so it ought to be integrated over some spacetime volume $\mathcal{M}$. Let this be the volume contained by two Cauchy surfaces $\Sigma_{2}$ (future-pointing) and $\Sigma_{1}$ (past-pointing), so that the boundary of $\mathcal{M}$ is:

$$
\partial \mathcal{M}=\Sigma_{2} \cup \Sigma_{1} \cup S,
$$

where $S$ are the timelike walls of the container, located at spatial infinity; see Figure 3.1. Now we integrate dJ and apply Stokes' theorem:

$$
\begin{aligned}
0=\int_{\mathcal{M}} \mathrm{dJ} & =\int_{\partial \mathcal{M}} \mathrm{J} \\
& =\int_{\Sigma_{2}} \mathrm{~J}+\int_{\Sigma_{1}} \mathrm{~J}+\int_{\mathrm{S}} \mathrm{J} \\
& =\mathrm{Q}\left(\mathrm{t}_{2}\right)-\mathrm{Q}\left(\mathrm{t}_{1}\right)+\Phi\left(\mathrm{t}_{2}, \mathrm{t}_{1}\right),
\end{aligned}
$$

where the minus sign for $Q\left(t_{1}\right)$ comes because the normal of $\Sigma_{1}$ points towards the past rather than future, and we just named the integral over $S$ as the flux $\Phi$. This equation is really just global conservation of charge:

$$
\Delta \mathrm{Q}\left(\mathrm{t}_{2}, \mathrm{t}_{1}\right)+\Phi\left(\mathrm{t}_{2}, \mathrm{t}_{1}\right)=0
$$




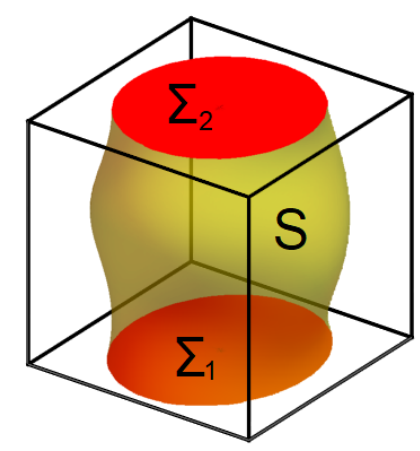

Figure 3.1: The boundary of the spacetime manifold $\mathcal{M}$ consists of two spacelike Cauchy surfaces $\Sigma_{2}$ and $\Sigma_{1}$ with a timelike surface $S$ located at infinity enclosing the two.

Classically, we would say the universe is a closed system, hence the flux $\Phi$ at infinity vanishes and $Q$ is constant. Quantum mechanically, a similar conclusion applies. The following commutator must vanish:

$$
\left\langle 0\left|\left[\Delta \mathrm{Q}\left(\mathrm{t}_{2}, \mathrm{t}_{1}\right)+\Phi\left(\mathrm{t}_{2}, \mathrm{t}_{1}\right), \mathrm{A}(\mathrm{y})\right]\right| 0\right\rangle=0
$$

for some operator $A(y)$ evaluated inside $\mathcal{M}$. But $\Phi$ is a boundary integral at spatial infinity; thus $[\Phi, A]=0$ due to the causal properties of operators in a quantum field theory. Hence we have $\langle 0|[\Delta Q, A]| 0\rangle=0$. So the quantum mechanical equivalent of charge conservation is:

$$
\langle 0|[Q(t), A(y)]| 0\rangle=C,
$$

for constant $C$.

SPACETIME SYMMETRIES. Notice that crucial for this proof was that the conserved current had the structure of a 3 -form, so that we could apply Stokes' theorem. In $(1+3) \mathrm{D}$, this means the conserved current must be a vector. However, spacetime symmetries don't necessarily admit this structure; for example, stress-energy is a symmetric tensor, so in general $\nabla_{\mu} T^{\mu \nu}=0$ doesn't imply energymomentum $\mathrm{P}^{\mu}$ is globally conserved. An exception is if a Killing 
vector $\xi_{\mu}$ exists, in which case we can define the stress-energy vector $j^{\mu} \stackrel{\text { def }}{=} \xi_{\nu} T^{\mu \nu}$. This vector is conserved because of Killing's equation, so energy-momentum is globally conserved in, e.g., Minkowski spacetime, but not in an arbitrary one.

\subsection{FOR BROKEN SYMMETRIES}

\subsubsection{Goldstone's theorem}

Goldstone's theorem refers to the existence of massless excitations on top of a vacuum that breaks global symmetries [30]. Noether's theorem still applies; the action is still symmetric, just the vacuum $10\rangle$ that isn't. So the previous result for global charge conservation must still hold. However, the requirement $Q|0\rangle \neq 0$ implies $\langle 0|[Q(t), A]| 0\rangle$ should not vanish in general. Hence the constant $C$ must be strictly nonzero.

Now let us expand the charge in terms of its density, i.e., $Q(t)=$ $\int d^{3} x j^{0}(t, \vec{x})$. Furthermore, for internal exact symmetries, it must be the case that the current has no explicit dependence on spacetime, in which case the usual Heisenberg relations,

$$
\frac{d j^{0}}{d x^{\mu}}=i\left[P_{\mu}, j^{0}\right]
$$

are solved by $j^{0}(x)=e^{i x^{\mu} P_{\mu} j^{0}}(0) e^{-i x^{\mu} P_{\mu}}$. For convenience of notation we are denoting the Hamiltonian $\mathcal{H}$ by $\mathrm{P}_{0}$. Using this, we can expand the matrix element:

$$
\langle 0|[Q(t), A]| 0\rangle=\int d^{3} x\left\langle 0\left|e^{i x^{\mu} P_{\mu}} j^{0}(0) e^{-i x^{\mu} P_{\mu}} A\right| 0\right\rangle-\text { h.c.. }
$$

Let us now insert the identity via,

$$
1=\sum_{n} \int d^{3} \vec{p}\left|n_{\vec{p}}\right\rangle\left\langle n_{\vec{p}}\right|
$$

where $\left|n_{\vec{p}}\right\rangle$ is an eigenstate of $\vec{P}$ (and thus also of $P_{0}$ since they commute) and $n$ represents any remaining labels of the state. Then: 


$$
\begin{aligned}
& =\sum_{n} \iint d^{3} x d^{3} p\left\langle 0\left|j^{0}(0) e^{-i x^{\mu} P_{\mu}}\right| n_{\vec{p}}\right\rangle\left\langle n_{\vec{p}}|A| 0\right\rangle-\text { h.c., } \\
& =\sum_{n} \iint d^{3} x d^{3} p\left\langle 0\left|j^{0}(0) e^{-i E_{n}(\vec{p}) t-i \vec{x} \cdot \vec{p}}\right| n_{\vec{p}}\right\rangle\left\langle n_{\vec{p}}|A| 0\right\rangle-\text { h.c., } \\
& =\lim _{\vec{p} \rightarrow \overrightarrow{0}} \sum_{n} e^{-i E_{n}(\vec{p}) t}\left\langle 0\left|j^{0}(0)\right| n_{\vec{p}}\right\rangle\left\langle n_{\vec{p}}|A| 0\right\rangle-\text { h.c., }
\end{aligned}
$$

where for the last line we performed the $\vec{x}$-integral, which converts $e^{-i \vec{x} \cdot \vec{p}}$ into $\delta(\vec{p})$, then performed the $\vec{p}$-integral. The result has to be constant, which requires $E_{n}(\overrightarrow{0})=0$ so that all timedependence drops from the expression. At the same time, it can't be zero. In particular, the following matrix element,

$$
\left\langle 0\left|j^{\mu}(\vec{p})\right| n_{\vec{p}}\right\rangle \neq 0,
$$

which represents our Goldstone state and justifies the assertion that the current creates a Goldstone out of the vacuum. Of course, if there are multiple broken internal symmetries, parametrized by some quantum numbers, the argument can be repeated for each broken charge, giving massless Goldstones with the same quantum numbers [31].

SPACETIME SYMMETRIES. Goldstone's theorem requires that the vacuum be invariant under translations and the charge have no explicit spacetime-dependence. Breaking the Poincaré group violates either the first assumption (for broken translations) or the second (for broken boosts/rotations), so Goldstone's theorem always fails.

\subsubsection{Fabri-Picasso theorem}

This final theorem refers to the phenomenon of superselection [32]. That is to say, not only are the vacua degenerate, but they live in different sectors of the S-matrix, and no physical process can map 
one vacuum into the other. Because of this, there's no sense in which one can build a superposition of all the vacua, such that the superposition doesn't break the symmetry.

First, it's useful to express the charge as a surface integral. For a spacetime of trivial de Rham cohomology, charge conservation can be directly solved:

$$
\mathrm{d} \mathrm{J}=0 \Longrightarrow \mathrm{J}=\mathrm{dF},
$$

for some 2 -form $F$. The charge is the integral of the current over some Cauchy surface $\Sigma$ :

$$
\mathrm{Q}=\int_{\Sigma} \mathrm{J}=\int_{\Sigma} \mathrm{dF}=\oint_{\partial \Sigma} \mathrm{F}
$$

using Stoke's theorem. Now, consider the norm of the state $Q|0\rangle$ :

$$
\begin{aligned}
\left\langle 0\left|Q^{2}\right| 0\right\rangle & =\oint_{\partial \Sigma} d^{2} x n_{i}\left\langle 0\left|F^{0 i}(x) Q(t)\right| 0\right\rangle \\
& =\oint_{\partial \Sigma} d^{2} x n_{i}\left\langle 0\left|e^{i x \cdot P} F^{O i}(0) e^{-i x \cdot P} e^{i x \cdot P} Q(0) e^{-i x \cdot P}\right| 0\right\rangle \\
& =\left\langle 0\left|F^{O i}(0) Q(0)\right| 0\right\rangle \oint_{\partial \Sigma} d^{2} x n_{i} \\
& =\left\langle 0\left|F^{O r}(0) Q(0)\right| 0\right\rangle \times 4 \pi R^{2}
\end{aligned}
$$

where $n_{\mu}$ is a vector normal to $\partial \Sigma, F^{O r}$ is the time-radial component of the 2-form $F$ in spherical coordinates, $R$ is the characteristic length of the system, and again we assumed the charge had no explicit spacetime-dependence in order to expand $Q(t)$. Unless $\mathrm{Q}|0\rangle=0$-in which case the above is zero-the norm of $\mathrm{Q}|0\rangle$ diverges as the system's size tends to infinity. Thus, the state $Q|0\rangle$ can't be part of the Hilbert space where $|0\rangle$ lives. More generally, for $\mathrm{D}$ spacetime dimensions, the result diverges as $\mathrm{R}^{\mathrm{D}-2}$. Notice that for $\mathrm{D}=2$, no superselection occurs, thus there is no true symmetry breaking. 
Spacetime symmetries. Since the Fabri-Picasso required no explicit time-dependence for $Q(t)$, it too can fail for spacetime symmetries. A counterexample to the theorem is the DBI action [33] in $\mathrm{D}=2$ dimensions: as it's constructed by embedding a $2 \mathrm{D}$ flat brane in $3 \mathrm{D}$ flat bulk, some of the boosts of the bulk-whose charges depend on time-will be broken. But according to the theorem there should be no spontaneous symmetry breaking in $\mathrm{D}=2$. 
Part II

TOOLS 



\section{COSET CONSTRUCTION}

\section{I INTRODUCTION}

If a symmetry is unbroken, its presence in the action is manifest, but it doesn't inform us much about the degrees of freedom in the theory. For example, simply knowing our theory contains fields transforming under unbroken $\mathrm{SU}(\mathrm{N})$ doesn't tell their representation. On the other hand, broken symmetries aren't manifest-meaning it's not immediately obvious the action displays them-but can constrain the nature of the degrees of freedom simply because there aren't that many different ways the action can exhibit this symmetry.

Thus the basic recipe we want is to take the symmetry pattern of unbroken and broken symmetries, process it through an algorithm, and output the most general effective field theory displaying that pattern. The machinery for this was first developed for internal symmetries in $1969[9,10]$.

This procedure-which goes by the name of coset construction for reasons we will see momentarily-is thus an essential tool for any physicist interested in constructing effective field theories. It allows one to build the most general action from merely from knowledge of the abstract group-theoretical symmetry breaking pattern.

\section{Outline}

This chapter is structured as:

- In Section 4.2 we explain how symmetry breaking patterns and Goldstone bosons are intimately related to homogeneous spaces which lack a preferred choice of origin. These concepts converge towards coset spaces from group theory, so that 
Goldstones inherit the powerfully binding group structure which constrains the form their action can take.

- In Section 4.3 we present a novel manner to complete the coset construction and derive the invariant action in a theory by normalization of transformed quantities.

- In Section 4.4 we review the usual manner in the literature through which the coset construction is completed, by computation of the distinguished Maurer-Cartan form, and relate this method to the normalization procedure.

- In Section 4.5 we discuss quasi-symmetries that shift the action by a boundary term and how to construct operators that display this property.

\subsection{HOMOGENEOUS SPACES}

Arguably, the most important property of a Goldstone boson $\pi$ is that the action of the broken symmetry transforms one state without bosons into one with them:

$$
\pi=0 \rightarrow \pi \neq 0 \text {. }
$$

This property of the group action is called transitivity. We are familiar with transitive group actions from maximally symmetric sacetimes. For instance, in Minkowski space, translations can move the origin to any point. In de Sitter space under the usual flat slicing coordinates,

$$
\mathrm{d} \mathrm{s}^{2}=-\mathrm{dt} \mathrm{t}^{2}+\mathrm{e}^{2 \mathrm{Ht}} \mathrm{d} \overrightarrow{\mathrm{x}}^{2}
$$

translations of space together with spacetime dilation $t \rightarrow t+$ $\lambda / H, \vec{x} \rightarrow e^{\lambda} \vec{x}$ can freely move the origin as well. Intuitively, all the points of a maximally symmetric spacetime are identical; it does not matter what one calls the origin. Such spacetimes are homogeneous. ${ }^{1}$

I But not all homogeneous spacetimes are maximally symmetric, as they may lack isotropy. 
Objects transforming transitively are not in a representation, since representations are linear: a matrix acting on the zero vector returns the zero vector. In other words, they can't move the origin. Because of this, these kinds of transformations are often said to be nonlinear. ${ }^{2}$

Let $S$ denote the spacetime generators that can move the spacetime origin, that is, for which the coordinates $x$ transform nonlinearly. Let $A$ be broken generators, with corresponding Goldstone bosons $\pi$ also transforming nonlinearly. If there is no intersection between the $S$ 's and the A's, we can collect the corresponding $x^{\prime}$ s and the $\pi^{\prime}$ 's into a single space $Q\{x, \pi\}$ for which the symmetry group $\mathrm{G}$ still acts transitively. Such a space is called a homogeneous space.

Note that, in the space $Q$, the $\pi$ is just an additional coordinate on the same footing as $x$, not a field with spacetime dependence. Solving the equations of motion consists of finding the subspace embedded by $\pi(x)$ that minimizes the action.

Now, this seems to be mostly nomenclature, but homogeneous spaces are actually very constraining when building a theory, because they are mostly unique. To construct a homogeneous G-space, it suffices to know all symmetries that do not move the origin of $Q$, meaning that leave the spacetime origin and the vacuum invariants. These symmetries form the stability subgroup $S$ of $Q$. Then the orbit-stabilizer theorem establishes that Q corresponds to a coset space:

$$
\mathrm{Q} \sim \frac{\mathrm{G}}{\mathrm{S}}
$$

where $\sim$ means equivalence in the sense that it preserves the group product (i.e., homeomorphism), but not necessarily any additional structure $\mathrm{Q}$ might have. Concretely, this means that any

2 Care must be taken with the term "nonlinear transformation". Take the example of de Sitter space above but in conformal coordinates, $\tau=-e^{-H t} / \mathrm{H}$. The time $\tau$ now transforms as $\tau \rightarrow e^{-\lambda} \tau$. This is linear in the functional sense, but not in the group action one since it moves the origin of time $\tau_{0}=-1 / \mathrm{H}$. Whenever we say nonlinear transformation in this thesis, we always mean the latter, grouptheoretical meaning. 
$q \in Q$ can be written in terms of some group element $\ell s \in \mathrm{G}$ acting on the origin of $Q$, where $\ell \in G$ is called a lift (or coset space representative) and $s \in S$ is an arbitrary stability element.

So for each element in $Q$ we can associate an element in $G$, up to some $S$-ambiguity, so informally $Q \sim G / S$. But a left coset space comes equipped with a group action by left multiplication of $\mathrm{g} \in \mathrm{G}$ :

$$
\ell S \rightarrow \tilde{\ell} S=g \ell S .
$$

Thus, if one specifies the full symmetry group $\mathrm{G}$ and the stability subgroup $S$, then the transformation laws of all coordinates and fields follow automatically, which by extension fixes all invariants that can be used to build an action, as we will see shortly. This construction-connecting physical entities in $Q$ to a coset space in order to derive invariants-goes by the name of coset construction.

Notice the lack of overlap between the S's and the A's is crucial for the space to be homogeneous. For example, say we have a time translation $\mathrm{P}_{0}$, broken with corresponding boson $\pi$. To move the origin of time $t$, we must apply $P_{0}$, yet to shift $\pi$ out of its vacuum, we must also apply $P_{0}$. We are thus not free to move both $t$ and $\pi$ as we wish. The group action is no longer transitive, and the space $Q$ is no longer homogeneous. Its structure, transformation laws, and uniqueness are no longer guaranteed simply from the symmetries. This is why these kinds of symmetry breaking patterns are much harder to handle and lead to less constrained theories.

\section{$4 \cdot 3$ NORMALIZATION PROCEDURE}

Representing our objects as the lift of a coset space endows them with canonical transformation laws directly from the symmetry breaking pattern. It remains to see how one construct invariants out of these laws.

The basic idea is intuitive: any quantity that can be transformed away by the symmetry group clearly can't be an invariant. But the group is finite, so there is a finite number of quantities that can be transformed this way. Anything that remains afterwards is an invariant. This method is based on [34, 35]; readers interested in formal proofs of these results can check those references. 
As a quick example, consider the position $\vec{x}$ of a particle with Galilean relativity, with time coordinate $t$. Time translation can always set $t$ to zero, and so too can space translation with $\vec{x}$. Then there's the velocity $\dot{\vec{x}}$, but it too can be transformed away through Galilean boosts. This leaves us with the acceleration $\ddot{\vec{x}}$ and the rotations. But no amount of rotating can set $\ddot{\vec{x}}$ to zero; at most, we can align it with one axis. Then the component along this axis is $|\ddot{\vec{x}}|$; all others are zero. This is final invariant of the particle: the magnitude of its acceleration.

In this example, notice the appearance of velocities and accelerations. This is natural. The coset space is a homogeneous space and thus by transitivity anything in it can be eliminated. So if we want more transforming quantities than there are group parameters, we necessarily must extend the coset space with additional objects, the derivatives of our fields. We know how coordinates and fields transform, so we know how derivatives transform by chain rule.

Hence, the logic is as follows. We take our original homogeneous space $Q=\{x, \pi\}$ and extend it with a finite amount of derivatives $\partial_{x} \pi, \partial_{x}^{2} \pi$, and so on. We then transform those quantities under some special $g_{*} \in G$ to be determined later. These transformed objects are denoted with capital letters rather than tildes (e.g., $X$ instead of $\tilde{x})$ due to their special status as putative invariants.

Transforming $\left(x, \pi, \partial_{x} \pi \ldots\right)$ under the $g_{*}$, we schematically have:

$$
\begin{gathered}
x \stackrel{g_{*}}{\rightarrow} X, \\
\pi \stackrel{g_{*}}{\rightarrow} \Pi, \\
\frac{\partial \pi}{\partial x} \stackrel{g_{*}}{\rightarrow} \frac{d \Pi}{d X^{\prime}}, \\
\frac{\partial^{2} \pi}{\partial x^{2}} \stackrel{g_{*}}{\rightarrow} \frac{d^{2} \Pi}{d X^{2}},
\end{gathered}
$$

1. We start by normalizing $X$ and $\Pi$ to zero, ${ }^{3}$ which allows us to solve for some of the parameters of $g_{*}$. If this completely fixes

3 We can set it to any constant without affecting the result. For clarity of notation, we set it to zero. 
$g_{*}$, then $\mathrm{d} \Pi / \mathrm{dX}$ are the invariants of the theory, ${ }^{4}$ and we're done.

2. If not, we then attempt to normalize as many of the $\mathrm{d} \Pi / \mathrm{d} X$ to zero as possible, which lets us fix more of the $g_{*}$. If $g_{*}$ is completely fixed by now, then the remaining $\mathrm{d} \Pi / \mathrm{dX}$ are the invariants we're after. If none remain, then the $d^{2} \Pi / d X^{2}$ are the invariants.

3. If $g_{*}$ still hasn't been fixed, we repeat the procedure, setting as many of the $d^{2} \Pi / d X^{2}$ to zero as possible, and so on. In the end, when $g_{*}$ is completely determined (which can always be done since the group is finite-dimensional, so a finite number of normalizations fixes all parameters), the lowest order in derivatives $d^{n} \Pi / d X^{n}$ that survived the process are the invariants.

Next, in order to build an integration measure, we also need invariant 1 -forms. This is straightforward: we take $d x$, evaluate it under the $g_{*}$ found above, then use multiple exterior products to build a volume form $\mathrm{dV}$ :

$$
\begin{aligned}
\mathrm{d} x & \stackrel{\mathrm{g}_{*}}{\rightarrow} \mathrm{d} X, \\
\mathrm{dV} & =\operatorname{det}(\mathrm{d} X), \\
& =\frac{1}{\mathrm{D} !} \mathrm{d} X^{0} \wedge \mathrm{d} X^{1} \wedge \ldots \wedge \mathrm{d} X^{\mathrm{D}} .
\end{aligned}
$$

The invariants together with the measure give the complete set of objects needed to build a strictly invariant action for the Goldstones of some symmetry breaking pattern.

Notice that in this procedure, we had to fix all parameters of $g_{*}$, including those of the stability subgroup $S$. This can end up being laborious. However, when the lift is properly parametrized, all coordinates and fields will transform in a linear representation of $S$. Therefore, invariance under $S$ is achieved just by writing objects in a manifestly $S$-invariant manner. What this means concretely is that we don't need to fix these parameters of $g_{*}$ coming from $S$.

4 Notice that setting $\Pi=0$ doesn't imply $\mathrm{d} \Pi=0$. 
Refraining from doing so means that all objects we derive during this procedure are covariant under S, and will be fully G-invariant when taking proper contractions of indices.

THE GALILEAN PARTICLE, I. To make the example quicker, let's consider the dimension one case. Upon transforming time $t$, position $x$ and velocity $v=\dot{x}$ we get the putative invariant o-forms and 1 -forms:

$$
\begin{array}{rlrl}
\mathrm{T} & =\mathrm{t}+\mathrm{s}, \quad \mathrm{X} & =\mathrm{x}+\mathrm{d}+\mathrm{bt}, \quad \mathrm{V} & =\mathrm{v}+\mathrm{b}, \\
\mathrm{d} T & =\mathrm{dt}, \quad \mathrm{dX}=\mathrm{d} x+\mathrm{bdt}, \quad \mathrm{d} V=\mathrm{d} v,
\end{array}
$$

where $s, d$ and $b$ are group parameters we need to eliminate by normalizing invariants. We start with the o-forms. Setting $\mathrm{T}=\mathrm{X}=\mathrm{V}=0$ kills everyone:

$$
s=-t \quad d=-x+v t \quad b=-v .
$$

Inserting the solution into the 1 -forms and recalling $x=x(t), v=$ $\dot{x}(t)$ results in the invariant forms:

$$
\mathrm{dT}=\mathrm{dt} \quad \mathrm{dX}=0 \quad \mathrm{dV}=\ddot{x} \mathrm{dt} .
$$

We see now that the invariant derivative is given by the linear dependence between $d T$ and $d V$ :

$$
\frac{d V}{d T}=\frac{d^{2} X}{d T^{2}}=\ddot{x} .
$$

The exactly invariant action for the Galilean particle then is:

$$
S=\int \mathrm{d} t \wedge \mathrm{F}\left(\frac{\ddot{x}}{\Lambda}\right)
$$

for some energy scale $\Lambda$ and arbitrary function $F$. 


\subsection{MAURER-CARTAN PROCEDURE}

Say we parametrize the coset lift as,

$$
\ell=e^{x \cdot S} e^{\pi \cdot A}
$$

By the normalization procedure, we must apply $g_{*}$ to $\ell S$ to compute the transformed lift, then normalize its components to zero in order to fix $g_{*}$. So:

$$
\begin{gathered}
\ell S \stackrel{g_{*}}{\rightarrow} \mathcal{L} S=g_{*} \ell S, \\
\mathrm{~d} \ell S \stackrel{g_{*}}{\rightarrow} \mathrm{d} \mathcal{L} S=g_{*} \mathrm{~d} \ell S,
\end{gathered}
$$

Normalizing $\mathcal{L S}=S$ means (4.20) is solved by $g_{*}=s \ell^{-1}$, where $s \in \mathrm{S}$. Assuming $x$ and $\xi$ are in an $S$-representation, we can suppress the s-part of $g_{*}$, so that all derived quantities are covariant rather than invariant.

We have now finished fixing $g_{*}$, so (4.2I) collects all covariants:

$$
\mathrm{dLS}=\ell^{-1} \mathrm{~d} \ell S .
$$

The 1 -form $\Omega=\ell^{-1} \mathrm{~d} \ell$ is distinguished and receives a special name: the Maurer-Cartan form. It's algebra-valued, so let us decompose it in terms of generators $S, A$ and $V$ :

$$
\Omega S=\left(\omega_{S} \cdot S+\omega_{A} \cdot A+\omega_{V} \cdot V\right) S=\left(\omega_{S} \cdot S+\omega_{A} \cdot A\right) S .(4 \cdot 23)
$$

Thus, after we project out $\omega_{V}$, the other two forms $\omega_{S}$ and $\omega_{A}$ are our remaining covariant 1 -forms. We must now extract covariant o-forms out of these. Let us decompose them into the spacetime and field bases $\mathrm{d} x$ and $\mathrm{d} \xi$ : 


$$
\begin{aligned}
& \omega_{S}^{\alpha}=\left(\omega_{S}^{\chi}\right)_{\mu}^{\alpha} \mathrm{d} x^{\mu}+\left(\omega_{S}^{\xi}\right)_{J}^{\alpha} \mathrm{d} \xi^{J} \\
& =\left[\left(\omega_{S}^{\chi}\right)_{\mu}^{\alpha}+\left(\omega_{S}^{\xi}\right)_{J}^{\alpha} \partial_{\mu} \xi^{J}\right] \mathrm{d} x^{\mu} \\
& \omega_{A}^{\mathrm{I}}=\left(\omega_{A}^{x}\right)_{\mu}^{\mathrm{I}} \mathrm{d} x^{\mu}+\left(\omega_{\mathrm{I}}^{\xi}\right)_{\mathrm{J}}^{\mathrm{I}} \mathrm{d} \xi^{J} \\
& =\left[\left(\omega_{A}^{x}\right)_{\mu}^{\mathrm{I}}+\left(\omega_{A}^{\xi}\right)_{J}^{\mathrm{I}} \partial_{\mu} \xi^{J}\right] \mathrm{d} x^{\mu} \\
& =\left[\left(\omega_{A}^{\chi}\right)_{\mu}^{\mathrm{I}}+\left(\omega_{A}^{\xi}\right)_{J}^{\mathrm{I}} \partial_{\mu} \xi^{\xi}\right]\left[\left(\omega_{S}^{\chi}\right)_{\mu}^{\alpha}+\left(\omega_{S}^{\xi}\right)_{K}^{\alpha} \partial_{\mu} \xi^{K}\right]^{-1} \omega_{S}^{\alpha}
\end{aligned}
$$

This establishes a linear dependence between the $\omega_{A}^{\mathrm{I}}$ forms and the $\omega_{S}^{\alpha}$ forms. Since these forms are covariant, so too are the linear coefficients. We thus get the following covariant quantities:

$$
K_{\alpha}^{\mathrm{I}}=\left[\left(\omega_{A}^{\chi}\right)_{\mu}^{\mathrm{I}}+\left(\omega_{\AA}^{\xi}\right)_{J}^{\mathrm{I}} \partial_{\mu} \xi\right]\left[\left(\omega_{S}^{\chi}\right)_{\mu}^{\alpha}+\left(\omega_{S}^{\xi}\right)_{K}^{\alpha} \partial_{\mu} \xi^{K}\right]^{-1} .
$$

Meanwhile, $\omega_{\mathrm{S}}$ can be used directly to build an invariant volume form:

$$
\begin{aligned}
\mathrm{dV} & =\operatorname{det}\left(\omega_{S}\right) \\
& =\frac{1}{\mathrm{D} !} \varepsilon_{\alpha \ldots}\left[\left(\omega_{S}^{\chi}\right)_{\mu}^{\alpha}+\left(\omega_{S}^{\xi}\right)_{J}^{\alpha} \partial_{\mu} \xi^{J}\right] \mathrm{d} x^{\mu} \wedge \ldots
\end{aligned}
$$

THE GALILEAN PARTICLE, II. The generator of time translations $P_{0}$, space shifts $Q$ and boosts $B_{0}$ admit the matrix representation:

$$
P_{0}=\left[\begin{array}{lll}
0 & 0 & 1 \\
0 & 0 & 0 \\
0 & 0 & 0
\end{array}\right] \quad Q=\left[\begin{array}{lll}
0 & 0 & 0 \\
0 & 0 & 1 \\
0 & 0 & 0
\end{array}\right] \quad B_{0}=\left[\begin{array}{lll}
0 & 0 & 0 \\
1 & 0 & 0 \\
0 & 0 & 0
\end{array}\right] \text {. (4.32) }
$$


Setting the coset lift $\ell=e^{t P_{0}} e^{x Q^{\nu B_{0}}}$ and computing the MaurerCartan form $\Omega=\ell^{-1} \mathrm{~d} \ell$ yields:

$$
\Omega=\left[\begin{array}{ccc}
0 & 0 & \mathrm{dt} \\
\mathrm{d} v & 0 & \mathrm{~d} x-v \mathrm{~d} t \\
0 & 0 & 0
\end{array}\right]
$$

or, decomposing in terms of the algebra generators,

$$
\omega_{\mathrm{P}}^{0}=\mathrm{dt} \quad \omega_{\mathrm{Q}}=\mathrm{d} x-v \mathrm{dt} \quad \omega_{\mathrm{B}}^{0}=\mathrm{d} v .
$$

These are precisely the same invariant 1 -forms we found before.

\subsubsection{Goldstone's theorem, revisited}

If all broken symmetries are internal, there can't be any mixing between spacetime and field sectors:

$$
\begin{aligned}
& \left(\omega_{S}^{\xi}\right)_{J}^{\alpha}=0 \\
& \left(\omega_{A}^{x}\right)_{\mu}^{I}=0,
\end{aligned}
$$

while $\left(\omega_{S}^{x}\right)_{\mu}^{\alpha}$ depends explicitly only on $x$ and $\left(\omega_{\mathrm{I}}^{\xi}\right)_{J}^{\mathrm{I}}$ only on $\xi$. The covariants then simplify to

$$
K_{\alpha}^{I}=\left(\omega_{\AA}^{\xi}\right)_{J}^{I}\left[\left(\omega_{S}^{\chi}\right)_{\mu}^{\alpha}\right]^{-1} \partial_{\mu} \xi
$$

For a metric spacetime ${ }^{5}$, the $\alpha \beta$-indices are contracted with the flat spacetime metric $\eta_{\alpha \beta}$, which means $\left(\omega_{S}^{\chi}\right)_{\mu}^{\alpha}$ has the interpretation of a tetrad of the world metric $g_{\mu \nu}$ :

$$
\eta_{\alpha \beta}\left(\omega_{S}^{\chi}\right)_{\mu}^{\alpha}\left(\omega_{S}^{\chi}\right)_{v}^{\beta}=g_{\mu v}(x) .
$$

5 Meaning the spacetime symmetries correspond to the isometries of some metric. A counterexample would be the Galileo group, which isn't the isometry group of any metric spacetime. 
Similarly, since all broken symmetries are internal, the IJ-indices are contracted with the flat internal metric $\delta_{\mathrm{IJ}}$, and $\left(\omega_{\AA}^{\xi}\right)_{\mathrm{J}}^{\mathrm{I}}$ is a tetrad of the field space metric $\mathrm{G}_{\mathrm{I}}$ :

$$
\delta_{\mathrm{IJ}}\left(\omega_{A}^{\xi}\right)_{\mathrm{K}}^{\mathrm{I}}\left(\omega_{A}^{x}\right)_{\mathrm{L}}^{\mathrm{J}}=\mathrm{G}_{\mathrm{KL}}(\xi) .
$$

Hence, the lowest order invariant of the constructions is:

$$
K^{2}=\delta_{I J} \eta^{\alpha \beta} K_{\alpha}^{\mathrm{I}} K_{\beta}^{\mathrm{J}}=\mathrm{G}_{\mathrm{IJ}}(\xi) g^{\mu \nu}(x) \partial_{\mu} \xi^{\mathrm{I}} \partial_{\nu} \xi^{\mathrm{J}} .
$$

Higher order invariants are built out of $\mathrm{K}_{\alpha}^{\mathrm{I}}$ and the metrics, plus possibly permutation symbols if we tolerate parity-odd operators. This means the Goldstones always appear carrying at least one derivative, and so all operators vanish at zero momentum. Furthermore, since they are all massless, no Goldstone can be integrated in favor of the other and all appear in the low energy theory. That is to say, there's no notion of inessential boson; we have one Goldstone for each broken generator, exactly.

This gives Goldstone's theorem in classical form. In fact, since $\xi$ is a spacetime scalar, the final expression for $K_{\mu}^{\mathrm{I}}$ is already diffeomorphic invariant and valid in curved spacetimes, where the spacetime metric $g_{\mu \nu}$ is arbitrary or even dynamical, while the computation of the field metric $\mathrm{G}_{\mathrm{IJ}}$ via the Maurer-Cartan form remains unchanged.

Note that since the final invariant is purely geometrical, requiring simply knowledge of the metrics $g_{\mu \nu}$ and $G_{I J}$, one can often be a bit clever and skip the entire procedure. If we know a metric space that is equivalent to the same coset space as our symmetry breaking pattern, we automatically know $\mathrm{G}_{\mathrm{IJ}}$. For instance suppose an antiferromagnet breaks $\mathrm{SO}(3)$ to $\mathrm{SO}(2)$. But notice that the symmetry group of the sphere is $\mathrm{SO}(3)$ and its origin, the north pole, has stabilizer subgroup $\mathrm{SO}(2)$; thus the sphere is equivalent to $\mathrm{SO}(3) / \mathrm{SO}(2)$, at least locally. ${ }^{6}$ So $\mathrm{G}_{\mathrm{IJ}}$ must necessarily be the metric

$6 \overline{\text { It's actually equivalent globally, }}$ but we don't need to prove this here, as we just need the metric $G_{I J}$ which is a local object. 
of the sphere! The fundamental invariant of the antiferromagnet (in flat spacetime) then is automatically fixed to be:

$$
K^{2}=(\partial \theta)^{2}+(\sin \theta)^{2}(\partial \varphi)^{2},
$$

for the Goldstones (or magnons) $\theta$ and $\varphi$ [36]. Notice these are relativistic magnons (their propagation speed is 1 ), as the spacetime symmetry group is Poincaré.

\subsection{QUASI-INVARIANTS}

So far, we have discussed only strict invariants, meaning quantities that are exactly invariant under the symmetry. But for the purposes of constructing an action, that might be a bit too strict: we can tolerate a Lagrangian that changes by a total derivative. Operators with this property are then quasi-invariants, or Wess-Zumino terms.

The basic idea is to take the various strictly invariant 1 -forms that are produced by the coset construction and wedge them together to form a $(D+1)$-form $\alpha$ that is exact, that is, $\alpha=d \beta$. Then the invariance of $\alpha$ implies:

$$
\delta \alpha=0 \Longrightarrow \mathrm{d} \delta \beta=0 \Longrightarrow \delta \beta=\mathrm{d} \gamma,
$$

for $\gamma$ a ( $D-1)$-form (trivial de Rham cohomology assumed). Now, if $\gamma=0$, then $\beta$ is exactly invariant and doesn't constitute anything original; it should follow directly as a product of the coset construction by wedging D invariant 1 -forms. But if $\gamma \neq 0$, then we have something genuinely new. And since $\beta$ is a D-form, the following term can be added to the exactly invariant action:

$$
S_{\mathrm{WZ}}=\int \beta
$$


THE GALILEAN PARTICLE, III. Recall we obtained three invariant 1 -forms for the Galilean particle in one dimension, from transforming time $t$, position $x$ and velocity $v$ :

$$
\mathrm{dT}=\mathrm{dt} \quad \mathrm{dX}=\mathrm{d} x-v \mathrm{dt} \quad \mathrm{dV}=\mathrm{d} v
$$

Let's try to wedge them into 2-forms and check if they are exact:

$$
\begin{aligned}
& \mathrm{dX} \wedge \mathrm{dT}=\mathrm{d} x \mathrm{dt}=\mathrm{d}(\mathrm{xdt}) \\
& \mathrm{d} V \wedge \mathrm{dT}=\mathrm{d} v \mathrm{dt}=\mathrm{d}(v \mathrm{~d} t) \\
& \mathrm{d} V \wedge \mathrm{dX}=\mathrm{d} v \mathrm{~d} x-v \mathrm{~d} v \mathrm{dt}=\mathrm{d}\left(v \mathrm{~d} x-\frac{1}{2} v^{2} \mathrm{dt}\right)
\end{aligned}
$$

Indeed, all are exact. So, the first one gives the tadpole $x \mathrm{dt}$, indicating a potential of constant gradient in the action doesn't break Galilean symmetry. The second is simply $\dot{x} \mathrm{dt}$; this is merely a total derivative and doesn't constitute anything new. The final one, upon inserting $\mathrm{d} x=\dot{x} \mathrm{dt}$, gives the kinetic energy $\dot{x}^{2} / 2 \mathrm{dt}$. These supplement the exactly symmetric action, to form the ultimate action for the Galilean particle:

$$
S=\int d t\left[\frac{1}{2} m \dot{x}^{2}-m g x+\Lambda f\left(\frac{\ddot{x}}{\Lambda}\right)\right]
$$

Now, to find all Wess-Zumino terms, one can certainly just wedge forms mindlessly in all possible combinations and check which ones are exact, but it would be useful to know a priori which wedges will be successful. For this, we must study the Lie algebra cohomology [37]. One can take the Maurer-Cartan form $\Omega$ and compute the structure equation:

$$
\mathrm{d} \Omega=-[\Omega \wedge \Omega] .
$$

We then decompose $\Omega=\omega_{S} \cdot S+\omega_{A} \cdot A+\omega_{V} \cdot V$ and wedge the $\omega_{S}$ 's and $\omega_{A}$ 's together in a manner that is invariant under the $V^{\prime} s$ (e.g., if $V$ are the Lorentz generators, then Lorentz indices must properly contracted). Upon using the structure equation, some 
of these wedge products may vanish, $\alpha=0$, which thus implies $\alpha=\mathrm{d} \beta$. We can then restrict our explicit computation to these $\beta$ only.

the galilean particle, iv. Computing $d \Omega$ gives:

$$
\mathrm{d} \Omega=\left[\begin{array}{ccc}
0 & 0 & 0 \\
0 & 0 & -\mathrm{dtd} \nu \\
0 & 0 & 0
\end{array}\right],
$$

which implies the structure equations, upon decomposing in terms of the algebra, are:

$$
\mathrm{d} \omega_{\mathrm{P}}^{0}=0 \quad \mathrm{~d} \omega_{\mathrm{Q}}=-\omega_{\mathrm{P}}^{0} \wedge \omega_{\mathrm{B}}^{0} \quad \omega_{\mathrm{B}}^{0}=0 .
$$

Looking at the following two 2-forms,

$$
\alpha_{1}=\omega_{\mathrm{Q}} \wedge \omega_{\mathrm{P}}^{0} \quad \alpha_{2}=\omega_{\mathrm{Q}} \wedge \omega_{\mathrm{B}}^{0}
$$

we see that for both of them $\mathrm{d} \alpha=0$ when using the structure equations, so $\alpha=\mathrm{d} \beta$. The $\beta^{\prime}$ 's then are our quasi-invariant 1forms.

Quasi-invariants are intimately related to powerfully predictive effective field theories, in the sense of having few free parameters. The fact an operator shifts by a total derivatives means it must appear exactly as-is in the action, not inside a free function. Because there are, at most, D quasi-invariants, an action constructed only from them (typically because the exact invariants carry undesirable higher derivatives) has a very limited number of allowed operators.

Note that once a symmetry is realized only up to total derivatives at the level of the Lagrangian, some of the results we have derived so far may no longer apply, necessarily. In particular, the conserved current of an internal quasi-symmetry may carry explicit spacetime dependence. To see this, suppose the Lagrangian is quasi-invariant under infinitesimal symmetry variation of fields $\delta \psi$ :

$$
\delta \mathcal{L}=\nabla_{\mu} \mathrm{K}^{\mu}
$$


Noether's conserved current is:

$$
j^{\mu}=\frac{\partial \mathcal{L}}{\partial \nabla_{\mu} \psi^{a}} \delta \psi^{a}-K^{\mu} .
$$

As long as $\mathcal{L}$ and $\delta \psi$ have no spacetime dependence, the first term in the current is spacetime-free as well. However, even for internal symmetries, the structure function $\mathrm{K}^{\mu}$ can depend on spacetime. For example, consider a single scalar field $\phi$ enjoying a shift symmetry $\delta \phi=c$. The tadpole $\phi$ is quasi-invariant:

$$
\delta \phi=\partial_{\mu}\left(\frac{c}{D} \chi^{\mu}\right)
$$

yet the structure function $\mathrm{K}^{\mu} \sim \chi^{\mu}$ depends on the coordinates explicitly. Once a current has such explicit dependence, the Heisenberg picture evolution $j(x)=e^{i x^{\mu} P_{\mu}}(0) e^{-i x^{\mu} P_{\mu}}$ fails and the quantum mechanical proof of Goldstone's theorem in Section 3.2.1 collapses.

\subsection{GAUge Symmetries}

For completeness, let us now briefly outline how the coset construction functions when the global group is gauged, that is to say, fibered over a base spacetime manifold. It is sufficient to show the usual gauge prescription in terms of covariant derivatives continues to work.

Recall that in the normalization procedure, we take all the quantities $\mathrm{q}$ in the coset space, whether coordinates and field, and transform them under some $g_{*} \in G$ into $Q$. Normalizing $Q$ to convenient values allows us to fix part of $g_{*} ;$ if is insufficient, we simply take the transformed 1 -forms dQ. Unfortunately, when $d g \neq 0$ as in a gauge group, this will fail, because dQ will introduce the derivatives of $g_{*}$ that now also need to be fixed.

Thus we simply the solution is exactly the same as the usual gauge prescription: promote the exterior derivative $d$ into the co- 
variant exterior derivative $\mathrm{D}$ such that $\mathrm{Dg}=0$. This is achieved by adding the connection 1 -form $A$ to $d$ :

$$
(d+A) u=0,
$$

$u \in G$. From this definition plus the fact $u$ transforms via the group product $u \rightarrow g u$, it follows that the transformation rule for $A$ is:

$$
\mathrm{A} \rightarrow \mathrm{gAg}^{-1}-\mathrm{dgg}^{-1} .
$$

Performing the normalization construction with this D then follows the same recipe. Obviously, one can also use the MaurerCartan form, in which case it's now computed via:

$$
\Omega=\ell^{-1} \mathrm{D} \ell
$$

for the coset space lift $\ell$. 


\section{$4 \cdot 7$ DISCUSSION}

The coset construction is a powerful suite of tools to construct the most general EFT associated with a given symmetry breaking pattern. Here we returned to first principles of the theory, presenting its connection to coset spaces and homogeneous spaces, which are essential for it to inherit the group-theoretical structure necessary for the machine to work.

We presented a novel way of performing the construction through normalization of the invariants. That is, we simply transform the objects living in the associated coset space, then normalize them and their derivatives to convenient values in order to fix the group parameter of this transformation. The remaining quantities are invariants. We then related this procedure to the usual one involving the Maurer-Cartan form.

Finally, we covered some extra remarks of importance in the coset construction. One was the construction of quasi-invariants which aren't exactly symmetric but shift by a total derivative- -hence, they are still permitted in the action. The second one was the issue of performing the construction when the global groups have been gauge. In this latter case, it was sufficient to show the usual gauge prescription of promoting derivatives to covariant derivatives are still the basic procedure one needs to do. 



\section{I INTRODUCTION}

In Section 4.6, we discussed one type of gauge symmetry, which are global symmetries gauged in the fiber bundle sense. The universe displays one other type of gauge symmetry, diffeomorphisms, which is a refined way of saying the mathematical structure of the laws of physics should be the same in all coordinate systems. General relativity in particular displays the largest possible set of diffeomorphisms:

$$
x^{\mu} \rightarrow f^{\mu}(x),
$$

which we denote by $\operatorname{Diff}(4)$. Note a diffeomorphism group is not a fiber gauge group, that is, it's not a global (or finite) group that has been gauged. This is because the symmetry acts on the base space of the fiber bundle. The rules of the game are therefore a bit different. When Sophus Lie first studied the subject of symmetry, he viewed the group as the same thing as transformations on some chosen space. That is, something like $\mathrm{ISO}(3)$ was defined by how it acts on Euclidean space. Nowadays, we see groups as abstract manifolds that don't need to refer to any other sort of space or construct. This is what allows us to define coset spaces solely from the abstract group product.

The problem is that, to the best of our current mathematical knowledge, diffeomorphism groups can't be defined as abstract entities [38]. They must always be represented by how they act on spacetime. This is why sometimes they are called pseudogroups, to distinguish them from true abstract groups. There's no sense in which we can "break" a diffeomorphism group into a subgroup and perform a coset construction because there are no cosets to begin with! 
But remember, the point of writing a coset element was simply to derive the transformation for the relevant objects in our theory. If we already have them from some other source-for instance, diff groups that are defined by how the coordinates transformthen we can simply apply the normalization procedure. Any group parameter that we fail to fix through the procedure can simply be declared to be a field. This is permitted because the group elements are spacetime-dependent functions, not abstract entities and they have a transformation rule inherited from the group product. We will see this in more detail momentarily.

\section{Outline}

We structure this chapter in the following way:

- In Section 5.2, we outline how to derive invariant objects for diffeomorphism groups in an algorithmically manner through the normalization procedure first described in Section 4.3.

- In Section 5.3, we explain how the description of a broken gauge symmetry requires introduction of new fields known as Stückelberg bosons.

- In Section 5.4, we apply these concepts to derive the effective field of cosmology through group theory rather than differential geometry, as it's usually done.

\subsection{NORMALIZATION PROCEDURE, REVISITED}

In Section 4.3, we introduced the normalization procedure for global groups (or global groups that have been gauged). It consisted of taking all objects $q$ that are transforming under the group $G$ (typically the transformation law is found by noticing these objects live in a coset space), and applying some $\mathrm{g}_{*} \in \mathrm{G}$ to transform the $q$ into putative invariants:

$$
\mathrm{q} \stackrel{g_{*}}{\rightarrow} \mathrm{Q},
$$


where $\mathrm{Q}$ are tentative invariants. Normalizing the Q's to zero (or some other convenient value) allows us to fix some of the parameters of $g_{*}$, and we can always continue the normalization by deriving $\mathrm{dQ}$, exploiting the fact $\mathrm{dg}=0$.

Of course, now the problem is that $d g \neq 0$, so computing $d Q$ gives us derivatives of $g_{*}$ that still need to be fixed. Performing the usual normalization construction should, fix $g_{*}$ in terms of $q$ and its derivatives, as before, but also in terms of derivatives of $g_{*}$ itself.

If the symmetry breaking pattern is "large", i. e., there are additional broken global symmetries, then typically we can fix the derivatives of $g_{*}$ in terms of the Goldstone bosons of the global breaking pattern. But if that's not the case, then we have no choice but to promote the derivatives of $g_{*}$ to fields whose transformation is inherited from the group product. In this case it's typically convenient to rewrite $\partial_{\mu} g^{\mathfrak{a}} \stackrel{\text { def }}{=} e_{\mu}^{\mathfrak{a}}$.

In this sense the problem is similar to that of gauging a global group in which we introduce Lie algebra-valued connection 1forms, that is to say, we are taking previously abstract objects and then using the fact they are now spacetime-dependent to promote then to fields. However, the transformation of a connection isn't inherited directly from the group product, but rather from the group parallel transport. This means the object $e_{\mu}^{a}$ is typically not a connection.

Here we give a simple example involving the geometry of planar curves with reparametrization invariance. For a more extended example, see Section 5.4 for the effective field theory of cosmology.

Curve geometry. Consider the $\operatorname{Diff}(1)$ group. It is defined by reparametrization of some $\lambda$ :

$$
\lambda \rightarrow f(\lambda) .
$$

By the normalization procedure, we simply declare the above to be putative invariants: 


$$
\begin{array}{r}
\Lambda=f(\lambda) \\
d \Lambda=f^{\prime}(\lambda) d \lambda .
\end{array}
$$

We can certainly normalize $\Lambda=0$ to fix $f(\lambda)$, but $f^{\prime}(\lambda)$ remains unfixed and there's nothing more we can do. We must simply declare $f^{\prime}=e$ to be a field, the "einbein", and that's it. The Diff(1)-invariant form is just $\int e \mathrm{~d} \lambda$.

Suppose now the problem is joined with that of the breaking of $\mathrm{ISO}(2) \rightarrow \mathrm{SO}(2)$, which describes planar curves, but now with diff invariance. These groups are global and usual coset techniques apply. We won't show it explicitly here for expediency, but the invariants associated with a curve $(x(\lambda), y(\lambda))$ are:

$$
\begin{aligned}
& \frac{d X}{d \Lambda}=\frac{x^{\prime} \cos \theta-y^{\prime} \sin \theta}{f^{\prime}(\lambda)}, \\
& \frac{d Y}{d \Lambda}=\frac{y^{\prime} \cos \theta+x^{\prime} \sin \theta}{f^{\prime}(\lambda)} .
\end{aligned}
$$

Putting $d X / d \wedge=0$ fixes $\tan \theta=y^{\prime} / x^{\prime}$. With this solution together with the normalization $\mathrm{d} Y / \mathrm{d} \Lambda=1$ we fix what we previously couldn't, $f^{\prime}(\lambda)=\sqrt{\left(x^{\prime}\right)^{2}+\left(y^{\prime}\right)^{2}}$. The Diff(1)-invariant line element is therefore:

$$
\mathrm{d} \Lambda=\sqrt{\left(x^{\prime}\right)^{2}+\left(y^{\prime}\right)^{2}} \mathrm{~d} \lambda .
$$

Of course, we could also proceed to derive the diffeomorphically invariant expression for the extrinsic curvature $\mathrm{k}$. 


\section{$5 \cdot 3$ STÜCKELBERG PROCEDURE}

One can wonder if there's any sense in which gauge symmetries can be broken. However, as we saw in Section 3.2.2, the associated conserved charge can be rewritten as a boundary integral:

$$
\mathrm{Q}=\oint_{\delta \Sigma} \mathrm{F},
$$

for $\Sigma$ a Cauchy surface and $F$ some 2-form. For small gauge transformations that vanish at infinity, the above is zero. Since the charge is also the symmetry generator, $Q=0$ implies the vacuum is always invariant, $Q|0\rangle=0$. These symmetries can't ever be broken, which makes sense since we interpret them as merely ambiguities in the mathematical formulation of the theory.

So there are no broken (small) gauge symmetries in the sense of a degenerate vacuum. But maybe there might still be some notion of Goldstone field. That is, if we "break" the gauge group $\mathcal{G} \rightarrow \mathcal{H}$, we search for some $\pi$ that transforms homogeneously under $\mathcal{H}$ but not under $\mathrm{G}$ :

$$
\begin{aligned}
& \pi=0 \stackrel{\mathcal{H}}{\rightarrow} \pi=0 \\
& \pi=0 \stackrel{\mathcal{G}}{\rightarrow} \pi \neq 0 .
\end{aligned}
$$

Naively, we would then want to say $\pi$ live in some coset space $\mathcal{G} / \mathcal{H}$. If $\mathcal{G}$ and $\mathcal{H}$ can be represented by global groups that have been gauged, then we can just take the global (and therefore abstract) versions of the groups, $G$ and $H$, and say that $\pi$ lives in $G / H$. Then we perform the coset construction on $\mathrm{G} / \mathrm{H}$ and gauge the resulting theory afterwards. For example, local $\mathrm{U}(1)$ isn't an abstract group, but obviously we can simply break global $\mathrm{U}(1)$ then gauge the result. But when the groups are diffeomorphism groups, they admit no representation as abstract groups. In particular, there are no such thing as a coset!

To our present understanding, the way one introduces Goldstone fields for broken diffs is via the Stückelberg procedure. Suppose we have constructed a theory invariant under $\mathcal{H}$, by using the 
techniques explained in this section. Diffeomorphism groups are defined by their action on the spacetime coordinates, so the action of $\mathcal{G}$ on $x^{\mu}$ is necessarily known and we can transform the action accordingly. Obviously, it changes, since it wasn't built to be $\mathcal{G}$-invariant to begin with. But since the group parameters $\xi$ are spacetime-dependent, not abstract group elements, we can simply promote them to spacetime fields: $\xi=-\pi$ (the minus sign is a convention). This new action, with this new degree of freedom $\pi$, is now invariant under $\mathcal{G}$, trivially so because $\pi$ has a transformation rule inherited from the group product that makes the action invariant.

The reason this works mathematically is because setting $\pi=0$ corresponds precisely to the action being just $\mathcal{H}$-invariant; hence, the condition $\pi=0$ is invariant under $\mathcal{H}$ but not under $\mathcal{G}$. The $\pi$ thus has the required transformation rule for a Goldstone nonlinearly realizing $\mathcal{G} \rightarrow \mathcal{H}$.

The reason this works physically is because gauge symmetries are just ambiguities in our description. Yes, we "created" a new degree of freedom by adding $\pi$, but we also introduced more gauge ambiguity that precisely eliminates these degrees of freedom. The Stückelberg procedure doesn't really do anything besides rewriting things in a different manner that is more convenient for low energy phenomenology.

One final disclaimer is about the uniqueness of this procedure. The coset construction guarantees that the Goldstones living in $\mathrm{G} / \mathrm{H}$ are the unique way to nonlinearly realize $\mathrm{G} \rightarrow \mathrm{H}$, up to field redefinitions. This is because of the orbit-stabilizer theorem. However, there are no cosets for diffeomorphism groups, nor is it there a version of the orbit-stabilizer theorem. So we don't know if the Stückelberg procedure is the unique way, up to field redefinitions, of nonlinearly realizing broken diffeomorphisms. Nevertheless, we don't know of any counterexample.

from proca to maxwell. The Proca mass term isn't invariant under local $\mathrm{U}(1)$ :

$$
A_{\mu} \rightarrow A_{\mu}+\partial_{\mu} \theta
$$


Declaring $\theta=-\pi$ to be a field now, we see its transformation follows from the $U(1)$ group product: $\delta \pi=-\lambda$. Since $\delta A_{\mu}=\partial_{\mu} \lambda$, the Proca mass is now invariant, and the Proca vector has become a Maxwell gauge vector.

\subsection{EXAMPLE: EFFECTIVE THEORY OF COSMOLOGY}

Cosmology is the study of the universe's evolution at the largest scales. By the Copernican principle, no location or direction in space is privileged, but there is a notion of absolute time. Concretely, this means that instead of displaying the full Diff(4) group, cosmology has a reduced diffeomorphism group defined by its action on spacetime:

$$
\begin{aligned}
& t \rightarrow t \\
& \vec{x} \rightarrow \vec{f}(t, \vec{x}) .
\end{aligned}
$$

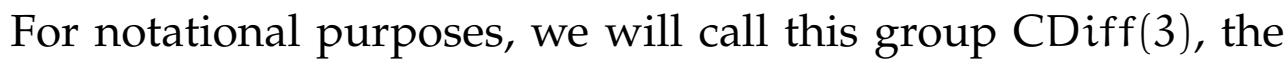
cosmology diffeomorphism group. One way to derive the invariants of such a group is to recognize that it induces a preferring slicing of the spacetime manifold and then use differential geometry to list by exhaustion all the objects associated with the embedding of these slices in the bulk [4]. Let's perform an alternative, group-theoretical derivation.

By the normalization procedure, we transform $t \rightarrow T=t$ and $x \rightarrow X$. The invariant 1 -forms then are:

$$
\begin{aligned}
\mathrm{d} X^{\mu} & =J_{v}^{\mu} \mathrm{d} x^{v} \\
J_{v}^{\mu} & =\partial_{v} f^{\mu},
\end{aligned}
$$

where of course $f^{0}=t$. We can't set $T$ to anything, as it doesn't transform (giving an algebraic invariant), and setting $X=0$ is insufficient to fix the Jacobian $J_{v}^{\mu}$. Since $J_{v}^{\mu} \stackrel{\text { def }}{=} e_{v}^{\mu}$ isn't an abstract group element but actually spacetime-dependent, we can simply use it as a brand new field. Our objects now transform: 


$$
\begin{aligned}
\mathrm{t} & \rightarrow \mathrm{t} \\
\vec{x} & \rightarrow \overrightarrow{\mathrm{f}}(\mathrm{t}, \vec{x}) \\
e_{v}^{\mu} & \rightarrow \mathrm{J}_{\rho}^{\mu} e_{v}^{\rho} .
\end{aligned}
$$

Actually, it's more convenient to put two e's together and define a new object with corresponding transformation rule:

$$
\begin{aligned}
& g^{\mu \nu}=e_{\rho}^{\mu} e_{\sigma}^{\nu} \eta^{\rho \sigma} \\
& g^{\mu \nu} \rightarrow J_{\rho}^{\mu} J_{\sigma}^{\nu} g^{\rho \sigma} .
\end{aligned}
$$

As should surprise nobody, this definition implies $g^{\mu \nu}$ is symmetric. Now, for the normalization procedure, we are interested in transforming $\mathrm{dt}, \mathrm{d} x^{i}$ and $g^{\mu \nu}$ under $\operatorname{CDiff}(3)$ to obtain putative invariants:

$$
\begin{aligned}
\mathrm{d} \mathrm{T} & =\mathrm{d} \mathrm{t} \\
\mathrm{d} X^{\mathrm{i}} & =\mathrm{J}_{\nu}^{\mathrm{i}} \mathrm{d} x^{\nu} \\
\mathrm{G}^{\mu \nu} & =\mathrm{J}_{\rho}^{\mu} J_{\sigma}^{\nu} \mathrm{g}^{\rho \sigma} .
\end{aligned}
$$

Normalization. The idea is that we normalize as many of the $\mathrm{G}^{\mu \nu}$ as possible to fix the Jacobian. Once that is fixed, we obtain four invariant derivatives by inverting the 1 -forms $d X^{\mu}$.

$$
\mathrm{d} \mathrm{X}^{\mu}=\mathrm{J}_{\nu}^{\mu} \mathrm{d} \mathrm{x}^{\nu} \Longrightarrow \mathrm{D}_{\mu}=\left[\mathrm{J}^{-1}\right]_{\mu}^{v} \partial_{\nu} .
$$

It proves convenient to parametrize the Jacobian in the following manner:

$$
J_{v}^{\mu}=\left[\begin{array}{cc}
1 & 0 \\
J_{0}^{i} & J_{j}^{i}
\end{array}\right]=\left[\begin{array}{cc}
1 & 0 \\
0 & R_{j}^{i}
\end{array}\right]\left[\begin{array}{cc}
1 & 0 \\
0 & u_{j}^{i}
\end{array}\right]\left[\begin{array}{cc}
1 & 0 \\
\beta^{i} & \delta_{j}^{i}
\end{array}\right],
$$

where $R_{j}^{i}$ is a rotation matrix and $U_{j}^{i}$ upper triangular. We choose the following normalization of $\mathrm{G}^{\mu \nu}$ :

$$
G^{\mu \nu}=\left[\begin{array}{cc}
g^{00} & 0 \\
0 & \delta^{i j}
\end{array}\right],
$$


which is sufficient to fix $U_{j}^{i}$ and $\beta^{i}$. Nothing can transform $g^{00}$, so it's invariant. The rotation $R_{j}^{i}$ remains unfixed. Nonetheless, since all objects under consideration transform linearly under $\mathrm{SO}(3)$, we can refrain from fixing it by performing manifestly $\mathrm{SO}(3)$-invariant contractions. In other words, the indices $i, j$ are covariant.

Derivatives. With the above normalization, we insert the solution for the Jacobian back into our derivative operators, with the caveat that $D_{i}$ is now covariant, so must be contracted with $\delta^{i j}$ :

$$
\begin{aligned}
D_{t} \psi & =g^{0 \mu} \partial_{\mu} \psi \\
\left(D_{x} \psi\right)^{2} & =\left(g^{i j} g^{00}-g^{i 0} g^{j 0}\right) \partial_{i} \psi \partial_{j} \psi .
\end{aligned}
$$

Each is invariant by itself, so any combination is also invariant. Using the fact $g^{00}$ is also invariant, we can take the Lorentz combination:

$$
g^{00}\left(D_{t} \psi\right)^{2}+\left(D_{x} \psi\right)^{2}=g^{\mu \nu} \partial_{\mu} \psi \partial_{\nu} \psi
$$

Hence, instead of using $D_{t}$ and $D_{x}$, we can use $D_{t}$ and $\partial_{\mu}$; the latter must be contracted in a manifestly Lorentz manner. ${ }^{1}$ The Lagrangian then is:

$$
\mathcal{L}\left(g^{\mu \nu} ; \partial_{\mu}, D_{t}, t\right)
$$

where Lorentz indices must be properly contracted using $g^{\mu \nu}$ and the derivative operators can only act on $\operatorname{CDiff}(3)$ scalars (e.g., $D_{t} g^{\mu \nu}$ is forbidden). Since $t$ is the only such scalar, let's see what its derivatives are:

$$
\begin{aligned}
& D_{t} t=g^{00} \\
& \partial_{\mu} t=\delta_{\mu}^{0} .
\end{aligned}
$$

1 In terms of differential geometry, using $D_{x}$ instead of $\partial_{\mu}$ means constructing objects that live on the equal-time slices, instead of the spacetime bulk. The fact either choice is sufficient is a manifestation of the Gauss-Codazzi relations. 
The first line, $D_{t} t=g^{00}$, justifies our omission of $g^{00}$ in the Lagrangian, as it was already included implicitly. Another quantity that is also implicit but nevertheless noteworthy is:

$$
n_{\mu}=\frac{\partial_{\mu} \mathrm{t}}{\sqrt{D_{\mathrm{t}} \mathrm{t}}}=\frac{\delta_{\mu}^{0}}{\sqrt{-\mathrm{g}^{00}}}
$$

where the normalization factor sets the magnitude of $n_{\mu}$ to - 1 . Remember the rules: $\partial_{\mu}$ must be contracted in a manifestly Diff(4) manner to preserve the $\operatorname{CDiff(3)}$ invariance of the scalar it acts on. This means that we must still contract $n_{\mu}$ as if it were a four-vector, even though the end result will not be Lorentz invariant.

Notice that by using $n_{\mu}$, we are free to replace $D_{t}$ by the Lie derivative $L_{n}$ along $n^{\mu}$. The advantage of doing this is that the Lie derivative preserves tensorial structure, so now we can take derivatives of the metric via $L_{n} g_{\mu \nu}$. Actually, $n^{\mu}$ isn't a true vector, so Lorentz contractions of $L_{n} g_{\mu \nu}$ will end up being merely CDiff(3)-invariant. Similarly, we can also take Lie derivatives of $n_{\mu}$ along itself.

A special combination of Lie derivatives of $g_{\mu \nu}$ and $n_{\mu}$ receives the name of extrinsic curvature:

$$
K_{\mu \nu}=\frac{1}{2} L_{n}\left(g_{\mu \nu}+n_{\mu} n_{v}\right),
$$

but it's not a new object, of course. It was already included given $g^{\mu \nu}, D_{t}$ and $t$.

Measure. We still need a volume element. That's simple: just wedge the four $\mathrm{d}^{\mu^{\mu}}$ s and insert the solution to the Jacobian. The result is:

$$
d V=d^{4} x \sqrt{g^{00} \operatorname{det} g}
$$

but since $g^{00}$ is already invariant, we might as well just remove it from the volume element.

Connection. There's one final consideration. Recall we decomposed the Jacobian in (5.26) in terms of a rotation $R_{j}^{i}$ and an uppertriangular matrix $u_{j}^{i}$. We fixed only $u_{j}^{i}$ in terms of the metric, 
arguing that we just needed to make $\mathrm{SO}(3)$ contractions to deal with $R_{j}^{i}$. This is true. However, remember that $R_{j}^{i}$ is a spacetimedependent matrix, not an abstract group element, so we could've added it as additional fields for the procedure! In turns out these act as a $3 \mathrm{D}$ connection $\Gamma_{j \mathrm{k}}^{\mathrm{i}}$ for the ${ }_{3} \mathrm{D}$ covariant derivative $\nabla_{i}^{(3)}$. Using $t$ and $L_{n}$, we can rewrite $\nabla_{i}^{(3)}$ in terms of the ${ }_{4} \mathrm{D}$ covariant derivative $\nabla_{\mu}$.

Output. Therefore, the final end result of the construction are the following elements:

$$
g_{\mu \nu}, \quad d^{4} x \sqrt{-g}, \quad t, \quad \nabla_{\mu}, \quad L_{n} .
$$

\subsubsection{In terms of the Stückelberg field}

We can follow the Stückelberg procedure to endow the cosmology EFT with the full Diff(4) group. In this way, cosmology can then be seen as a spontaneous symmetry breaking of general relativity. A diffeomorphism in $\operatorname{Diff}(4)$ but not in $\operatorname{CDiff}(3)$ takes the form:

$$
\begin{aligned}
& t \rightarrow t+\xi^{0}(t, \vec{x}), \\
& \vec{x} \rightarrow \vec{x},
\end{aligned}
$$

and then we promote $\xi^{0}=\pi$. Of the elements we derived in the previous section, $g_{\mu \nu}$ and $\nabla_{\mu}$ are already Diff(4)-covariant, and $d V$ is invariant. Meanwhile, the normal vector $n_{\mu}$ (and by extension, the Lie derivate) changes:

$$
n_{\mu} \rightarrow u_{\mu}=\frac{\partial_{\mu}(t+\pi)}{\sqrt{-g^{\mu \nu} \partial_{\mu}(t+\pi) \partial_{\nu}(t+\pi)}}
$$

Therefore the following elements,

$$
g_{\mu \nu}, \quad d^{4} x \sqrt{-g}, \quad t+\pi, \quad \nabla_{\mu}, \quad L_{\mathfrak{u}},
$$

are complete and sufficient for building an action that nonlinearly realizes $\operatorname{Diff}(4) \rightarrow \operatorname{CDiff}(3)$. 
Let's make this more concrete by studying what the action looks like. For simplicity, let's restrict ourselves to only first derivatives of $\pi$ in the action. Then the Lagrangian is:

$$
\mathcal{L}=\sum_{n \geqslant 0} \frac{b_{n}(t+\pi)}{n !}\left[\partial(t+\pi)^{2}\right]^{n}
$$

Now we can understand why this form is more convenient for phenomenology. Remember we want the vacuum $\pi=0$ to be a valid solution to the equations of motion, which means the Lagrangian cannot contain terms linear in $\pi$ (tadpoles). In view of this, it's convenient to rewrite the Lagrangian as:

$$
\begin{aligned}
\mathcal{L}=b_{0}(t+\pi)+b_{1}(t+\pi)\left[-1-2 \dot{\pi}+(\partial \pi)^{2}\right] & \\
& +\sum_{n \geqslant 2} \frac{d_{n}(t+\pi)}{n !}\left[\partial(t+\pi)^{2}+1\right]^{n},
\end{aligned}
$$

which follows from reshuffling the sum and redefining $b_{n}$ for $n \geqslant 2$ to extract all tadpoles; the remaining sum starts quadratic in $\pi$ since $\partial(t+\pi)^{2}+1=(\partial \pi)^{2}-2 \dot{\pi}$. So the terms linear in $\pi$ are:

$$
\pi\left(\dot{\mathrm{b}}_{0}-\dot{\mathrm{b}}_{1}\right)-2 \dot{\pi} \mathrm{b}_{1} .
$$

This has to be zero. Recall the metric determinant is $\sqrt{-g}$; since the above tadpole is first order in perturbation, we are only interested right now in the background determinant, so $a(t)^{3}$ for any homogeneous and isotropic cosmology. Now we can integrate by parts the above tadpole to mutate $\dot{\pi}$ into $\pi$, yielding:

$$
\pi\left(\dot{\mathrm{b}}_{0}+\dot{\mathrm{b}}_{1}+6 \mathrm{Hb} \mathrm{b}_{1}\right),
$$

where $\mathrm{H}$ is the Hubble parameter, $\mathrm{H}=\dot{\mathrm{a}} / \mathrm{a}$. Setting the above to zero then constrains the first coefficient of the EFT, "for free"! This is called tadpole cancellation, and its far more transparent here in terms of the Stückelberg field than with just the metric. Note 
that tadpole cancellation is model-independent, that is, we haven't invoked the Friedmann equations (or something else if general relativity has been modified).

If we insert this constraint back into the Lagrangian of (5.43), we obtain after some algebra an expression that starts manifestly at quadratic order in $\pi$ :

$$
\mathcal{L}=V_{0}(t)+\sum_{n \geqslant 2} \frac{1}{n !}\left[\lambda_{n}(t) \pi^{n}+d_{n}(t+\pi)\left((\partial \pi)^{2}-2 \dot{\pi}\right)^{n}\right]
$$

where

$$
\begin{aligned}
& V_{0}(t)=6 \int^{t} d s[H(s)-H(t)] b_{1}(s), \\
& \lambda_{n}(t)=6 H \frac{d^{n-1}}{d t^{n-1}}\left(b_{1}\right)-6 \frac{d^{n-1}}{d t^{n-1}}\left(H b_{1}\right) .
\end{aligned}
$$

Imposing the FLRW evolution of the vacuum $\pi=0$ allows us to fix the vacuum energy $V_{0}(t)$. That is, the first Friedmann equation reads:

$$
3 \mathrm{M}_{\mathrm{Pl}}^{2} \mathrm{H}^{2}=\mathrm{V}_{0}
$$

Taking the time derivative of the above yields the integral equation:

$$
M_{P l}^{2} H=-\int^{t} d s b_{1}(s)
$$

the solution of which is $b_{1}=-M_{\mathrm{Pl}}^{2} \dot{H}$. 


\section{$5 \cdot 5$ DIsCUSSION}

In this chapter we outlined how to derive the invariants for a diffeomorphism group. Such a group isn't abstract and its elements are spacetime-dependent. Such qualities fundamentally change the problem, though the normalization procedure we introduced can still be used. The key is precisely to employ the fact the elements are spacetime-dependent to promote them to fields where necessary.

We also discussed the role of Stückelberg bosons as the equivalent of a Goldstone boson for broken diffs, that is, as the fields that transform inhomogeneously (or nonlinearly) under the broken diffs. Introducing these degrees of freedom is, to our present knowledge, how broken diffeomorphism are realized.

As a concrete example, we re-derived the effective field theory of cosmology. This theory is the one built out of space reparametrizations that can depend on space and time together with an absolute notion of time. That is to say, it's the central symmetry group of cosmology, as the expansion of the universe provides a notion of absolute time direction. This theory has been constructed before using differential geometry, but here we provided a group-theoretical algorithm to do so. 
Part III

CASE STUDIES 



\subsection{INTRODUCTION}

Inflation is a period of near de Sitter acceleration and is currently the favored model to describe the origin of primordial fluctuations in the early universe [21]. These are responsible for seeding all perturbations in cosmology we see today, such as the cosmic microwave background and the large scale structure of matter. In particular, inflation is adept at explaining two key features of these primordial fluctuations: its near scale invariance and adiabaticity.

The field that sources this accelerated expansion is a scalar known as the inflaton. The most common type of inflaton theory is one that generates slow roll inflation, meaning the scalar background evolves slowly across its potential. But scalar fields are typically vulnerable to naturalness issues, as displayed also by the Higgs field [39]. If the cutoff of the inflaton theory is $\Lambda$, with $M_{P l}>\Lambda>H$, its mass receives quantum corrections of order $\Lambda$. Furthermore, degrees of freedom with mass higher than $\Lambda$ manifest themselves as higher dimensional operators that further modify the shape of the potential. These corrections then ruin the slow roll potential necessary for inflation to persist for a sufficient period of time, the so-called $\eta$ problem [40].

Of course, pragmatically, there is no issue whatsoever with this. One simply tunes the bare parameters of the potential against the quantum corrections in order to match observations. However, if we need to retune the bare parameters at each order in perturbation theory, then it would seem to suggest that UV physics is being constrained by IR physics, which, while not forbidden in principle, is still quite vexing [41].

A relative simple way of taming these quantum corrections is through a mere shift symmetry which the corrections themselves have to satisfy all the way to the quantum gravitational scale $M_{\mathrm{Pl}}$. 
Concretely, this means flattening the potential. Unfortunately, this rises more questions that it answers. It's generally believed that no quantum gravitational theory can have global symmetries (e.g., throw global charge carriers into a hairless black hole and you destroy the charge forever). This means any model with global symmetries would then live in the swampland of theories that aren't UV-completable [42-44]

Tackling the problem from an effective field theory (EFT) point of view allows us to determine, concretely, what the consequences of a shift symmetry for inflation are, while dodging all the issues discussed above. This lets us to consider all models of single scalar inflation together, without any bias or preselection. The structure of the additional shift symmetry then lets us make predictions for all these theories. In short, we can derive observational consequences that must be satisfied if a shift symmetry has anything to do with inflation, in any form. One of they main tools for this is the interplay between spontaneously broken symmetries and the infrared structure of theories, which leads to various theorems the primordial spectrum of fluctuations must satisfy.

Formalizing the nature of shift symmetries in inflation also lets us scrutinize some statements found in the literature. For example, in the standard slow roll EFT [4], the parameters of the theory, which in principle can be time-dependent, are taken to be roughly constant by invoking an approximate shift symmetry. As we will see, these arguments are less robust than at first glance.

Cosmology is a gravitational theory and as such diffeomorphisms are its key symmetries, so we will invoke many of the tools we discussed in Chapter 5. Even the shift symmetry must be treated in this manner-it may be global, but it nontrivially mixes with the pseudogroup of diffeomorphisms. Actually, since much of the discussion uses these generic symmetry tools, it can also be applied to other subjects of cosmology that might also involve a shift symmetry, such as superfluid dark matter [45] and dark energy $[46,47]$. 


\section{Outline}

This chapter follows this structure:

- In Section 6.2, we introduce the idea of broken time translation mixed with broken internal symmetries, in particular shifts. In flat spacetime, this breaking pattern governs various condensed matter systems. We then move on to apply the same principle for curved spacetimes, but now in terms of broken diffeomorphisms.

- In Section 6.3, we reintroduce the effective field theory of cosmology, first discussed in Section 5.4, then use the concept of the broken time diffeomorphism mixed with the broken shift to derive recursive constraints for the EFT parameters.

- In Section 6.4, we discuss the consequences of the infrared structure of the EFT of cosmology in the presence of the shift symmetry. This includes the presence of additional adiabatic modes, classical solutions produced by the broken diffeomorphism that are genuinely physical despite looking like mere gauge modes, as well as soft theorems, which constrain correlators where one mode is of much lower momentum than the others.

- In Section 6.5, we conclude the EFT of shift-symmetric cosmology by studying some phenomenology of a certain class of models included in the EFT. In the inflation literature, these models are part of the so-called ultra slow inflation class of models.

\subsection{BROKEN TIME TRANSLATIONS}

\subsubsection{Non-gravitational systems}

Condensed matter systems, being nonrelativistic, can be seen as possessing Galilean symmetry. Yet the universe is fundamentally Lorentzian, so a more accurate statement is that these systems do possess Lorentz boosts as a symmetry, but it's spontaneously 
broken. After all, a condensed substance selects a preferred frame of reference: its own rest frame.

In practice, all known condensed matter systems also break additional Poincaré symmetries. In particular, time translations are broken, because of the rest mass of a system's constituents. For example, consider the air in a room. The excited states of this system are described by sound waves (or phonons) on top of a "vacuum" state, which is not a literal vacuum but merely the absence of sound. But air molecules still have mass in this vacuum; the total air in the room still has a total energy given by $\mathrm{mN}$ where $m$ is the average mass of a molecule and $N$ the number of them. This seems immaterial, but remember that energy sources gravity, so the absolute energy of the vacuum does matter!

To make things more complicated, the air molecules still undergo thermal fluctuations, so that the vacuum also has a nonzero thermal energy. Worse, sound waves can't propagate without dissipative effects, so we can't even talk about energy being conserved. Let's then go the world of superfluids at zero temperature, where things propagate losslessly and no thermal excitations arise. This means we could define a new Hamiltonian:

$$
\overline{\mathcal{H}}=\mathcal{H}-\mathrm{mN},
$$

subtracting the inaccessible mass-energy stored in the molecules. Since $\mathcal{H}$ is conserved and $\mathrm{N}$ too (assuming no chemistry that can convert air molecules into other things), $\overline{\mathcal{H}}$ is conserved as well. And while $\mathcal{H}$ and $\mathrm{N}$ are broken, the overall combination $\overline{\mathcal{H}}$ is not $[31,48]$. The existence of the conserved $\overline{\mathcal{H}}$ then allows us to see the system as being in equilibrium: if time evolution is now defined by $\overline{\mathcal{H}}$, then the vacuum is static.

This forms the most basic symmetry breaking pattern for condensed matter, the superfluid. More generically, one has a chemical potential $\mu$ and an internal charge $\mathrm{Q}$ so that $\overline{\mathcal{H}}=\mathcal{H}-\mu \mathrm{Q}$ is unbroken [31]. The biggest advantage is that working in terms of $\overline{\mathcal{H}}$ instead of $\mathcal{H}$ is really just a basis change at the level of the abstract Lie algebra of the symmetry. That is to say, we can describe the superfluid symmetry breaking pattern uniquely in group-theoretical 
terms. This allows the coset construction described in Chapter 4 to be immediately applied to them without issue, and the most generic superfluid EFT can be readily constructed [49].

Other breaking patterns are possible as well. Solids for example break space translations $\vec{P}$ together with additional internal symmetries $\vec{Q}$, such that $\vec{P}=\vec{P}-\mu \vec{Q}$ is unbroken. We won't discuss them here, but the important insight is that broken internal symmetries mix with broken spacetime symmetries, from which the bulk properties of condensed matter systems can be classified [50]. This also neatly explains why condensed matter systems violate Goldstone's theorem. After all, the theorem only applies to internal symmetries [51].

Trouble arises when both $\mathrm{P}_{0}$ and $\mathrm{Q}$ are conserved but broken, yet no spacetime-independent combination between them that leaves the vacuum invariant exists. For example, consider the driven superfluid:

$$
\mathcal{L}=\mathrm{P}(\mathrm{X})+\lambda \phi,
$$

where $X=-(\partial \phi)^{2} / 2$. Notice this Lagrangian has a shift quasisymmetry, $\delta \phi=\mathrm{Q} \bar{\phi}=1$. The equation of motion is:

$$
\ddot{\bar{\phi}}(\mathrm{P}, \mathrm{X}+2 \mathrm{XP}, \mathrm{XX})=\lambda \text {. }
$$

For $\lambda=0$, it is always solved by $\bar{\phi}=\mu$ t regardless of the choice of $\mathrm{P}(\mathrm{X})$. This then implies

$$
\mu \delta \bar{\phi}=\dot{\bar{\phi}} \Longrightarrow\left(\mathrm{P}_{0}-\mu \mathrm{Q}\right) \bar{\phi}=0,
$$

as required, so the system is in equilibrium. As soon as $\lambda \neq$ 0 , however, the above won't be true anymore and the system will be out of equilibrium, which makes sense since the external source term $\lambda \phi$ drives the system. The problem is now no group-theoretical description of this symmetry breaking pattern exists. Of course, it is true that

$$
\left(\mathrm{P}_{0}-\dot{\bar{\phi}}(\mathrm{t}) \mathrm{Q}\right) \bar{\phi}=0
$$


but this statement makes reference to spacetime. Defining a new symmetry generator $\overline{\mathrm{P}}_{0}=\mathrm{P}_{0}-\dot{\bar{\phi}}(\mathrm{t}) \mathrm{Q}$ is nonsensical at the level of the abstract Lie algebra. No coset can be constructed from this, so the coset construction can't be applied.

In some cases, additional symmetries may help us tame the problem. For example, suppose now that $P(X)=X$, that is:

$$
\mathcal{L}=-\frac{1}{2}(\partial \phi)^{2}+\lambda \phi
$$

Then the theory enjoys an additional shift quasi-symmetry $\delta \phi=$ $\mathrm{B}^{\mu} \phi=x^{\mu}$. The equation of motion is now solved by $\bar{\phi}=\lambda \mathrm{t}^{2} / 2+\mu \mathrm{t}$, which means the following holds:

$$
\left(P_{0}-\mu Q-\lambda B^{0}\right) \bar{\phi}=0,
$$

allowing us to define the new generator $\bar{P}_{0}=P_{0}-\mu Q-\lambda B^{0}$ in an abstract manner. Of course, in this case, $\mathrm{B}^{0}$ isn't an internal symmetry, so the system isn't quite a superfluid. And, in general, we won't have any such additional symmetry.

\subsubsection{EFT of cosmology}

We have broken time translations and broken shifts, but without any spacetime-independent combination between them that is unbroken. If the problem is we can't denote the symmetry breaking pattern in an abstract manner, then let us work with groups that aren't abstract: diffeomorphism groups. In particular, we are interested in broken time diffs, which leads us to the effective field theory of cosmology we saw in Section 5.4.

Let us recall the EFT of cosmology. If general relativity is the theory enjoying the full group $\operatorname{Diff}(4)$ of spacetime diffeomorphisms, cosmology is the theory with merely the $\operatorname{CDiff(3)}$ group of absolute time diffeomorphisms:

$$
\begin{aligned}
& t \rightarrow t, \\
& \vec{x} \rightarrow \vec{f}(t, \vec{x}) .
\end{aligned}
$$


The most general toolbox for building an action with this symmetry was found in (5.37) and consists of the objects:

$$
g_{\mu \nu}, \quad d^{4} x \sqrt{-g}, \quad t, \quad \nabla_{\mu}, \quad L_{n},
$$

where $n_{\mu}=\delta_{\mu}^{0} / \sqrt{-g^{00}}$. All Lorentz indices must be contracted properly. Now, of course we could consider arbitrary applications of the derivatives, such as $\mathrm{L}_{n}^{34} g_{\mu v}$, but in fact we are only interest in two:

$$
\begin{aligned}
\mathrm{L}_{n} t & =g^{00}, \\
\mathrm{~L}_{n}\left(g_{\mu \nu}+n_{\mu} n_{v}\right) & =2 K_{\mu \nu},
\end{aligned}
$$

because too many derivatives of the metric renders the action pathological. In addition to an Einstein-Hilbert term, we can build a Lagrangian $\mathcal{L}$ expanded in terms of $g^{00}$ and the $K_{\mu \nu}$ :

$$
\mathcal{L}=\sum_{n, m \geqslant 0} \frac{b_{n}^{\langle\mu \nu\rangle_{m}}(t)}{n ! m !}\left(g^{00}\right)^{n}\left\langle K_{\mu \nu}\right\rangle^{m},
$$

where to avoid overcrowding the expression with indices, we are denoting:

$$
\begin{aligned}
b_{n}^{\langle\mu v\rangle_{m}} & =b_{n}^{\mu_{1} \ldots \mu_{m} v_{1} \ldots v_{m}}, \\
\left\langle K_{\mu \nu}\right\rangle^{m} & =K_{\mu_{1} v_{1}} \ldots K_{\mu_{m} v_{m}} .
\end{aligned}
$$

For the purposes of phenomenology, it's more appropriate to expand the Lagrangian in terms of perturbations of geometrical objects around some background. So we rewrite the Lagrangian as:

$$
\mathcal{L}=\sum_{n, m \geqslant 0} \frac{b_{n}^{\langle\mu \nu\rangle_{m}}}{n ! m !}\left(-1+g^{00}\right)^{n}\left\langle\bar{K}_{\mu \nu}+K_{\mu v}\right\rangle^{m},
$$

or, expanding the binomial product, 


$$
\begin{aligned}
=\sum_{n, m \geqslant 0} \sum_{p \leqslant n} \sum_{q \leqslant m} & \frac{b_{n}^{\langle\mu v\rangle_{m}}}{n ! m !}\left(\begin{array}{c}
n \\
p
\end{array}\right)\left(\begin{array}{c}
m \\
q
\end{array}\right) \\
& \times(-1)^{n-p}\left\langle\bar{K}_{\mu v}\right\rangle^{m-q}\left(\delta g^{00}\right)^{p}\left\langle\delta K_{\mu \nu}\right\rangle^{q}
\end{aligned}
$$

Notice that the following is true for the $n, p$ summations:

$$
\sum_{n \geqslant 0} \sum_{p \leqslant n}=\sum_{p \geqslant 0} \sum_{n \leqslant p}=\sum_{p, \ell \geqslant 0}
$$

where we defined a new dummy index $\ell=n-p$. A similar argument holds for the $m, q$ summations, defining the new index $\mathrm{k}=\mathrm{m}-\mathrm{q}$. Taking the Lagrangian and expanding the binomial coefficients and shuffling summations as described yields:

$$
\begin{aligned}
\mathcal{L} & =\sum_{p, q \geqslant 0} \frac{1}{p ! q !}\left[\sum_{\ell, k>0} \frac{b_{\ell+p}^{\langle\mu v\rangle_{k+q}}}{\ell ! k !}(-1)^{\ell}\left\langle\bar{K}_{\mu \nu}\right\rangle^{k}\right]\left(\delta g^{00}\right)^{p}\left\langle\delta K_{\mu v}\right\rangle^{q} \\
& =\sum_{p, q \geqslant 0} \frac{d_{p}^{\langle\mu v\rangle_{q}}}{\ell ! k !}\left(\delta g^{00}\right)^{p}\left\langle\delta K_{\mu v}\right\rangle^{q}
\end{aligned}
$$

That is precisely the series expansion in terms of the fluctuations, with the d's the new coefficients. They are related to the original b's via:

$$
d_{p}^{\langle\mu v\rangle_{q}}=\sum_{\ell, k>0} \frac{b_{\ell+p}^{\langle\mu v\rangle_{k+q}}}{\ell ! k !}(-1)^{\ell}\left\langle\bar{K}_{\mu v}\right\rangle^{k}
$$

For completeness, we note that the extrinsic curvature of the FLRW background is given in terms of the scale factor and Hubble parameter by:

$$
\overline{\mathrm{K}}_{\mu \nu}=\mathrm{a}^{2} \mathrm{H}\left(\mathrm{g}_{\mu \nu}+\delta_{\mu}^{0} \delta_{v}^{0}\right)
$$


Before we move on to the next step, it proves convenient to rewrite some of these EFT coefficients in terms of parameters describing the cosmological background. Let us focus on the linear action:

$$
\mathcal{L}=\mathrm{d}_{0}(\mathrm{t})+\mathrm{d}_{1}(\mathrm{t}) \delta \mathrm{g}^{00}+\hat{\mathrm{d}}_{0}(\mathrm{t}) \delta \mathrm{K}_{\mu}^{\mu} .
$$

This is the only part of the EFT that contributes towards the background stress-energy tensor:

$$
\overline{\mathrm{T}}_{\mu \nu}=\left[\frac{-2}{\sqrt{-\mathrm{g}}} \frac{\delta(\sqrt{-\mathrm{g}} \mathcal{L})}{\delta \mathrm{g}^{\mu \nu}}\right]_{\delta g^{00}=\delta \mathrm{K}_{\mu \nu}=0} .
$$

Sourcing the FLRW background through this stress-energy, we impose the two Friedmann equations in order to fix:

$$
M_{\mathrm{Pl}}^{2} \mathrm{H}^{2} \varepsilon=-\mathrm{d}_{1}-\frac{\dot{\hat{\mathrm{d}_{0}}}}{2}, \quad 3 \mathrm{M}_{\mathrm{Pl}}^{2} \mathrm{H}^{2}=-\mathrm{d}_{0}-2 \mathrm{~d}_{1}+3 \mathrm{H} \hat{\mathrm{d}}_{0},
$$

with $\varepsilon \stackrel{\text { def }}{=}-\dot{H} / H^{2}$. Lastly, there's the speed of sound $c_{s}$ of scalar fluctuations. Once we impose the above conditions, we look at the corresponding Lagrangian for the Stückelberg field $\pi$. It starts at second order in $\pi$, as it has to if we want to interpret $\pi$ as a fluctuation around a background rather than sourcing the background. The quadratic terms are:

$$
\begin{aligned}
\mathcal{L} & =M_{\mathrm{Pl}}^{2} \dot{\mathrm{H}}\left(-\frac{1}{\mathrm{c}_{s}^{2}} \dot{\pi}^{2}+\frac{1}{\mathrm{a}^{2}}\left(\partial_{\mathrm{i}} \pi\right)^{2}+\varepsilon \mathrm{H}^{2}\right) \\
\frac{1}{\mathrm{c}_{s}^{2}} & =1+\frac{2 \mathrm{~d}_{2}}{\varepsilon \mathrm{M}_{\mathrm{Pl}} \mathrm{H}^{2}} .
\end{aligned}
$$

Now we have all the pieces on the board to actually tackle the shift symmetry, which we do in the next section. 


\subsection{CONSTRAINTS ON EFT PARAMETERS}

\subsubsection{Procedure}

One issue in building a field theory using only the metric as our degrees of freedom is that it's not immediately clear how to introduce internal symmetries. These are symmetries of the matter field $\phi$ which doesn't appear at all in the action, so how should the symmetry be realized?

\subsubsection{Cosmic time}

The key is that any field transformation looks like a diffeomorphism. Generically, a scalar transforms under an internal symmetry as:

$$
\delta \phi=f(\phi) .
$$

In fact, through a field redefinition $\phi \rightarrow \int f(\phi)$, we can always recast the symmetry as a shift $\delta \phi=c$, so we can set $f(\phi)=c$ without loss of generality. Now, any background $\bar{\phi}$ breaks the shift symmetry $Q$, but suppose it also breaks time translations (but not spatial ones), so $\bar{\phi}=\bar{\phi}(\mathrm{t})$. A covariant theory has an infinite diffeomorphism group of coordinate transformations, generated by the Lie derivative. For the scalar, the Lie derivative acts as:

$$
\mathrm{L}_{\xi} \phi=\xi^{\mu} \partial_{\mu} \phi .
$$

We see then that the combination of a specific diffeomorphism $\xi^{\mu}=\delta_{0}^{\mu} / \dot{\bar{\phi}}$ together with the charge $\mathrm{Q}$ leaves the vacuum invariant:

$$
\left(\mathrm{L}_{\xi}-\mathrm{Q}\right) \bar{\phi}=0 .
$$

This defines a notion of nonuniform time translation that is still a symmetry. Note that for this to make sense, $\bar{\phi}$ must be monotonic or the argument breaks down at $\dot{\bar{\phi}}=0$. So we can always take $\bar{\phi}$ to 
be invertible. It is then easy to see that the finite time transformation that represents this symmetry is:

$$
t \rightarrow \bar{\phi}^{-1}(\bar{\phi}(t)+c)
$$

Of course, there is a price to pay. This diffeomorphism is not an isometry of the metric, so the latter does change under it:

$$
\begin{aligned}
\delta g_{\mu \nu} & =\left(L_{\xi}-Q\right) g_{\mu \nu}=\xi^{\rho} \partial_{\rho} g_{\mu \nu}+2 g_{\rho(\mu} \partial_{v)} \xi^{\rho}, \\
& \left.=\frac{1}{\dot{\bar{\phi}}} \dot{g}_{\mu \nu}+2 g_{0(\mu} \delta_{v}^{0}\right)\left(\frac{-\ddot{\bar{\phi}}}{\ddot{\bar{\phi}}}\right),
\end{aligned}
$$

where we used the charge is internal, so it's impossible for the metric to transform under it, $Q g_{\mu \nu}=0$. At this level, it doesn't seem we have accomplished much other than shuffling the symmetry around. The key is that the effective theory is constructed precisely out of geometric objects satisfying all symmetries of the background $\bar{\phi}$.

While we want the effective field theory of cosmology to break arbitrary time diffeomorphisms, we want to impose that it should still be symmetric under this specific one. This means the Lagrangian should transform as a scalar under this diffeomorphism:

$$
\mathrm{L}_{\xi} \mathcal{L}=\xi^{\mu} \partial_{\mu} \mathcal{L}=\frac{c}{\dot{\bar{\phi}}}\left(\frac{\partial \mathcal{L}}{\partial \mathrm{t}}+\frac{\partial \mathcal{L}}{\partial g_{\mu \nu}} \dot{\boldsymbol{g}}_{\mu \nu}+\frac{\partial \mathcal{L}}{\partial \partial_{\rho} g_{\mu \nu}} \partial_{\rho} \dot{g}_{\mu \nu}\right) .
$$

At the same time, the Lagrangian should also transform according to the imposed metric transformation (6.33):

$$
\delta \mathcal{L}=\frac{\partial \mathcal{L}}{\partial g_{\mu \nu}} \delta g_{\mu \nu}+\frac{\partial \mathcal{L}}{\partial \partial_{\rho} g_{\mu \nu}} \partial_{\rho} \delta g_{\mu \nu}
$$

Equating $L_{\xi} \mathcal{L}=\delta \mathcal{L}$ then imposes a constraint on the coefficients of the Lagrangian. For clarity, it proves useful to first compute these constraints assuming no extrinsic curvature before performing the more complex computation with it. 


\subsubsection{Clock time}

As the inflaton background $\bar{\phi}(\mathrm{t})$ evolves monotonically in time, it selects a time coordinate $\tau$ in which its rate of change is constant: $\partial_{\tau} \bar{\phi}=\mu$. We relate this coordinate to the usual cosmic time $t$ via:

$$
\mu \mathrm{d} \tau=\dot{\bar{\phi}} \mathrm{d} t
$$

which is a pure time diffeomorphism, $t \rightarrow \tau(t)$. Now, the effective theory of cosmology isn't built to be invariant under these diffeomorphisms, so its mathematical form, including relations between coefficients once the internal shift is imposed, do change. In any case, the Lagrangian can still be constructed in this coordinate:

$$
\mathcal{L}=\sum_{n, m \geqslant 0} \frac{c_{n}^{\langle\mu \nu\rangle_{m}}(\tau)}{n ! m !}\left(g^{00}\right)^{n}\left\langle K_{\mu \nu}\right\rangle^{m} .
$$

We can repeat the same argument as in the previous section: find the diffeomorphism $L_{\xi}$ that leaves the background invariant:

$$
\left(\mathrm{L}_{\xi}-\mathrm{Q}\right) \bar{\phi}(\tau)=0,
$$

except now the answer is trivial: $\xi^{\mu}=c \delta_{0}^{\mu}$ since the background's evolution is constant. In short, the special diffeomorphism that corresponds to the shift symmetry in clock time is a uniform time translation. So imposing the shift symmetry is simply the requirement that all the c's be constant in time. The downside is that these coordinates aren't the usual ones for computing observables in cosmology; in particular, $\partial_{\tau} g^{00} \neq 0$ is inconvenient.

\subsubsection{Relations for $\mathcal{L}\left(\mathrm{g}^{00}\right)$}

Let's first start with the simpler case where the Lagrangian only contains $g^{00}$ terms. We work in cosmic time where the Lagrangian is:

$$
\mathcal{L}=\sum_{n \geqslant 0} \frac{b_{n}(t)}{n !}\left(g^{00}\right)^{n}
$$


and insert it in $\mathrm{L}_{\xi} \mathcal{L}$ :

$$
\begin{aligned}
\mathrm{L}_{\xi} \mathcal{L} & =\frac{1}{\dot{\bar{\phi}}} \dot{\mathcal{L}} \\
& =\frac{1}{\dot{\bar{\phi}}} \sum_{n \geqslant 0}\left[\frac{\dot{b}_{n}}{n !}\left(g^{00}\right)^{n}+\frac{n b_{n}}{n !} \dot{g}^{00}\left(g^{00}\right)^{n-1}\right] .
\end{aligned}
$$

In the sum, the term proportional to $\dot{g}^{00}$ vanishes for $n=0$, so we can shift the dummy index $n \rightarrow n+1$ :

$$
=\frac{1}{\dot{\bar{\phi}}} \sum_{n \geqslant 0}\left[\frac{\dot{b}_{n}}{n !}+\frac{b_{n+1}}{n !} \dot{g}^{00}\right]\left(g^{00}\right)^{n} .
$$

Meanwhile, we compute the variation of the Lagrangian $\delta \mathcal{L}$, using the transformation rule for $\mathrm{g}^{00}$ :

$$
\begin{aligned}
\delta \mathcal{L} & =\sum_{n \geqslant 0} \frac{n b_{n}}{n !}\left(g^{00}\right)^{n-1} \delta g^{00} \\
& =\frac{c}{\dot{\bar{\phi}}} \sum_{n \geqslant 0}\left[\frac{n b_{n}}{n !} \dot{g}^{00}\left(g^{00}\right)^{n-1}+\frac{2 \ddot{\bar{\phi}}}{\dot{\bar{\phi}}} \frac{n b_{n}}{n !}\left(g^{00}\right)^{n}\right]
\end{aligned}
$$

Again, the term proportional to $\dot{\mathrm{g}}^{00}$ can have its dummy index shifted, $n \rightarrow n+1$ :

$$
=\frac{c}{\dot{\bar{\phi}}} \sum_{n \geqslant 0}\left[\frac{b_{n+1}}{n !} \dot{g}^{00}+\frac{2 \ddot{\bar{\phi}}}{\dot{\bar{\phi}}} \frac{b_{n}}{n !}\right]\left(g^{00}\right)^{n} .
$$

Setting $L_{\xi} \mathcal{L}=\delta \mathcal{L}$ order by order in powers of $\left(g^{00}\right)^{n}$ enforces the relation:

$$
\dot{\mathrm{b}}_{\mathrm{n}}=\frac{2 \ddot{\bar{\phi}}}{\dot{\bar{\phi}}} \mathrm{n} \mathrm{b}_{\mathrm{n}} .
$$

We also need the relation for the $d_{n}(t)$, the coefficients of the expansion in terms of $\delta g^{00}$. We start with the relation between the $d_{n}$ and $b_{n}$, take a time derivative and insert (6.46): 


$$
\begin{aligned}
\dot{\mathrm{d}}_{\mathrm{n}} & =\sum_{m \geqslant 0} \frac{\dot{\mathrm{b}}_{\mathrm{m}+\mathrm{n}}}{\mathrm{m} !}(-1)^{\mathrm{m}} \\
& =\frac{2 \ddot{\bar{\phi}}}{\bar{\phi}} \sum_{m \geqslant 0} \frac{\mathrm{b}_{\mathrm{m}+\mathrm{n}}(\mathrm{m}+\mathrm{n})}{\mathrm{m} !}(-1)^{\mathrm{m}} \\
& =\frac{2 \ddot{\bar{\phi}}}{\dot{\bar{\phi}}}\left[-\sum_{m \geqslant 0} \frac{\mathrm{b}_{(\mathrm{m}-1)+(\mathrm{n}+1)}}{(\mathrm{m}-1) !}(-1)^{\mathrm{m}-1}+\mathrm{n} \sum_{\mathrm{m} \geqslant 0} \frac{\mathrm{b}_{\mathrm{m}+\mathrm{n}}}{\mathrm{m} !}(-1)^{\mathrm{m}}\right] \\
& =\frac{2 \ddot{\bar{\phi}}}{\dot{\bar{\phi}}}\left(n \mathrm{~d}_{\mathrm{n}}-\mathrm{d}_{\mathrm{n}+1}\right)
\end{aligned}
$$

Importantly, these relations are perturbatively invariant under renormalization group flow. Should we wish to compute quantum corrections to the various coefficients in the EFT, the relations must be preserved, as they follow from symmetry. ${ }^{1}$

We can also recast these relations in a different form to make its dependence on the FLRW background clearer. Recall that $d_{0}$ and $d_{1}$ are fixed by the FLRW background:

$$
\mathrm{M}_{\mathrm{Pl}}^{2} \mathrm{H}^{2} \varepsilon=-\mathrm{d}_{1}, \quad 3 \mathrm{M}_{\mathrm{Pl}}^{2} \mathrm{H}^{2}=-\mathrm{d}_{0}-2 \mathrm{~d}_{1} .
$$

At the same time, we have the $n=0$ relation that connects these two d's:

$$
\dot{\mathrm{d}}_{0}=-\frac{2 \ddot{\bar{\phi}}}{\dot{\bar{\phi}}} \mathrm{d}_{1} \text {. }
$$

Recognizing $\eta \stackrel{\text { def }}{=} \dot{\varepsilon} / \varepsilon H$ then lets us solve for the $\bar{\phi}$ term:

$$
\frac{2 \ddot{\bar{\phi}}}{\dot{\bar{\phi}}}=2 \mathrm{H}(3-2 \varepsilon+\eta),
$$

I We don't expect the symmetry to become anomalous at the quantum level, but it can be broken by nonperturbative effects. 
so that the recursive relations of the EFT coefficients become:

$$
\dot{d}_{n}=2 H(3-2 \varepsilon+\eta)\left(n d_{n}-d_{n+1}\right) .
$$

In the literature, it's not uncommon to assume the near constancy of the EFT parameters during slow roll inflation by evoking an approximate shift symmetry. Here we see this isn't well justified. The slow roll conditions $\varepsilon \ll 1$ and $|\eta| \ll 1$ are incompatible with $\dot{\mathrm{d}}_{\mathfrak{n}}=0$ ! We will have more to say about this in section Section 6.5.

\subsubsection{Relations for $\mathcal{L}\left(\mathrm{g}_{00}, \mathrm{~K}_{\mu \nu}\right)$}

We are now ready to tackle an action contain both the $g^{00}$ and $\mathrm{K}_{\mu \nu}$ terms. To avoid repeating most of the same arguments from the previous section, we perform the computation in a different manner, this time in clock time. For the clock time Lagrangian, imposing the shift symmetry simply means all coefficients in the theory must be constant in time:

$$
\begin{aligned}
\mathcal{L} & =\sum_{n, m \geqslant 0} \frac{c_{n}^{\langle\mu \nu\rangle_{m}}}{n ! m !}\left(g^{00}\right)^{n}\left\langle K_{\mu \nu}\right\rangle^{m}, \\
\partial_{\tau} c_{n}^{\langle\mu \nu\rangle_{m}} & =0 .
\end{aligned}
$$

This was easy. The hard part is transforming it to cosmic time, which is a more commonly used time parametrization. Recall we need to remap $\tau \rightarrow \tau(t)$, defined by:

$$
\mu \frac{\mathrm{d} \tau}{\mathrm{dt}}=\dot{\bar{\phi}}
$$

Transforming $g^{00}$ under this coordinate change is straightforward: $g^{00} \rightarrow \dot{\bar{\phi}}^{2} g^{00} / \mu^{2}$. The extrinsic curvature $K_{\mu \nu}$, however, is more complicated. It's not a tensor under time diffeomorphisms, so we must go back to its definition: 


$$
\begin{aligned}
K_{\mu v} & =\frac{1}{2} L_{n}\left(g_{\mu v}+n_{\mu} n_{v}\right) \\
n_{\mu} & =\frac{\delta_{\mu}^{0}}{\sqrt{-g^{00}}} .
\end{aligned}
$$

After some calculation, one can check the extrinsic curvature doesn't transform under purely time diffeomorphisms, $\mathrm{K}_{\mu \nu} \rightarrow \mathrm{K}_{\mu \nu}$. This is expected, since $K_{\mu \nu}$ measures the curvature that a constanttime slice of spacetime is endowed with due to this embedding. But purely time diffeomorphisms don't change the embedding or how we slice spacetime, merely the threading of the slices, that is, how we stack them together.

Therefore, the Lagrangian in usual cosmic time is:

$$
\begin{aligned}
\mathcal{L} & =\sum_{n, m \geqslant 0} \frac{\dot{\bar{\phi}}^{2 n}}{\mu^{2 n}} \frac{c_{n}^{\langle\mu v\rangle_{m}}}{n ! m !}\left(g^{00}\right)^{n}\left\langle K_{\mu v}\right\rangle^{m}, \\
& =\sum_{n, m \geqslant 0} \frac{b_{n}^{\langle\mu v\rangle_{m}}(t)}{n ! m !}\left(g^{00}\right)^{n}\left\langle K_{\mu v}\right\rangle^{m},
\end{aligned}
$$

that is to say, the b's of the theory in cosmic time are related to the c's of the clock time theory by:

$$
b_{n}^{\langle\mu v\rangle_{m}}=\frac{\dot{\bar{\phi}}^{2 n}}{\mu^{2 n}} c_{n}^{\langle\mu v\rangle_{m}}
$$

The requirement that $\partial_{\tau} c_{n}^{\langle\mu v\rangle_{m}}=0=\dot{c}_{n}^{\langle\mu v\rangle_{m}}$ then forces the following relation for the $b^{\prime} s$ :

$$
\dot{\mathrm{b}}_{\mathrm{n}}^{\langle\mu v\rangle_{m}}=\frac{2 \ddot{\bar{\phi}}}{\dot{\bar{\phi}}} \mathrm{nb} \mathrm{n}_{\mathrm{n}}^{\langle\mu v\rangle_{m}}
$$

Rewriting in terms of the d's using (6.21) leads to:

$$
\dot{\mathrm{d}}_{n}^{\langle\mu v\rangle_{m}}=\frac{2 \ddot{\bar{\phi}}}{\dot{\bar{\phi}}}\left(n d_{n}^{\langle\mu v\rangle_{m}}-d_{n+1}^{\langle\mu v\rangle_{m}}\right)+d_{n}^{\langle\mu v\rangle_{m+1}} \dot{\bar{K}}_{\mu_{m+1} v_{m+1}}
$$


In the previous section, we were able to recast $d_{0}$ and $d_{1}$ in terms of the FLRW background metric by using the Friedmann equations. Unfortunately, this won't work here as we have three d's but still only two equations:

$$
M_{\mathrm{Pl}}^{2} \mathrm{H}^{2} \varepsilon=-\mathrm{d}_{1}-\frac{\dot{\mathrm{d}_{0}}}{2}, \quad 3 \mathrm{M}_{\mathrm{Pl}}^{2} \mathrm{H}^{2}=-\mathrm{d}_{0}-2 \mathrm{~d}_{1}+3 \mathrm{H} \hat{\mathrm{d}}_{0} . \text { (6.65) }
$$

If we now try to solve for the $\bar{\phi}$ term, the best we manage to do is eliminate $d_{0}$ and $\hat{d}_{0}$ :

$$
\frac{2 \ddot{\bar{\phi}}}{\dot{\bar{\phi}}}=6 \mathrm{H}+2 \frac{\dot{\mathrm{d}}_{1}}{\mathrm{~d}_{1}}
$$

The recursive relations then aren't fully specified purely in terms of the background as $d_{1}$ acts as a free function:

$$
\dot{\mathrm{d}}_{\mathrm{n}}^{\langle\mu v\rangle_{\mathfrak{m}}}=\left(6 \mathrm{H}+2 \frac{\dot{\mathrm{d}}_{1}}{\mathrm{~d}_{1}}\right)\left(n \mathrm{~d}_{\mathrm{n}}^{\langle\mu v\rangle_{\mathfrak{m}}}-\mathrm{d}_{n+1}^{\langle\mu v\rangle_{\mathfrak{m}}}\right)+\mathrm{d}_{n}^{\langle\mu v\rangle_{m+1}} \dot{\bar{K}}_{\mu_{\mathfrak{m}+1} v_{\mathfrak{m}+1}} .
$$

\subsection{INFRARED STRUCTURE}

\subsubsection{Adiabatic modes}

For the purposes of computing observables in cosmology, we aren't particularly interested in working with the metric directly, but rather the scalar and tensor modes it contains. For instance, the scalar curvature fluctuations $\zeta$ generated by inflation in the early universe ultimately source temperature perturbations we observe in the CMB today. Similarly, tensor fluctuations $h_{i j}$ are expected to act as a primordial source of gravitational waves. These degrees of freedom are extracted from the metric via its ADM decomposition:

$$
\begin{aligned}
d s^{2}=- & (1+\alpha)^{2} d t^{2} \\
& +\left(a(t) e^{\zeta}\right)^{2}\left(\delta_{i j}+h_{i j}\right)\left(d x^{i}+\beta^{i} d t\right)\left(d x^{j}+\beta^{j} d t\right),
\end{aligned}
$$


where $\alpha$ and $\beta^{i}$ are called shift and lapse, respectively. For simplicity, we will set tensor fluctuations to zero, $h_{i j}=0$, and also assume the lapse is a scalar mode, $\beta_{i}=\partial_{i} \psi$. Spatial indices are lowered and raised via $\delta_{i j}$.

The shift and the lapse don't actually appear with time derivatives in the Einstein field equations. This means they aren't propagating degrees of freedom, but rather Lagrange multipliers, so that their equations of motions represent constraints for the theory. Inserting the solution to these constraints back in the action then produces a pure $\zeta$ action. To first order in perturbations, these constraints are:

$$
\begin{aligned}
\partial_{i}(\Theta \alpha-\dot{\zeta}) & =0, \\
\partial_{i} \partial_{j}\left(a^{2} \dot{\psi}+3 a^{2} H \psi+\alpha+\zeta\right) & =0,
\end{aligned}
$$

and are solved by:

$$
\begin{aligned}
& \alpha=\frac{1}{\Theta} \dot{\zeta}, \\
& \psi=\frac{F(\vec{x})}{a^{3}}-\frac{1}{a^{3}} \int^{t} d t^{\prime} a\left(t^{\prime}\right)\left(\alpha\left(t^{\prime}\right)+\zeta\left(t^{\prime}\right)\right),
\end{aligned}
$$

for $F(\vec{x})$ some arbitrary function and $\Theta$ a model-dependent parameter, equal to $\mathrm{H}$ if the scalar field action contains only first derivatives. $^{2}$

Now, the difficulty is that the symmetries of the action with the Lagrange multipliers won't necessarily be inherited by the pure $\zeta$ action. Only those that leave the constraints invariant will. This is a problem for our shift symmetry. The transformation for $\alpha, \beta^{i}$ and $\zeta$ are found by applying a diffeomorphism $\xi^{\mu}$ on the metric. Infinitesimally, they are:

2 For instance, if $\mathcal{L}=P(X)+G(X) \square \phi$ for $X=-(\partial \phi)^{2} / 2$, then $\Theta=H+$ $\dot{\bar{\phi}}^{3} \mathrm{G}_{, \mathrm{X}} / 2 \mathrm{M} \mathrm{Pl}$. 


$$
\begin{aligned}
\delta \alpha & =\dot{\xi}^{0}, \\
\delta \xi & =H \xi^{0}+\frac{1}{3} \partial_{i} \xi^{i}, \\
\delta \beta_{i} & =\dot{\xi}^{i}-\frac{1}{a^{2}} \partial_{i} \xi^{0} .
\end{aligned}
$$

We can then check that the shift diff $\xi_{\mu}=c / \dot{\bar{\phi}} \delta_{\mu}^{0}$ will not leave the constraint equations invariant, unless we are in the zero-momentum limit where all fields have no space dependence.

There's still hope. The shift diff failed to be a symmetry of $\zeta$ despite being a symmetry of the metric. There's the possibility that other diffs in CDiff(3) might also display the same issue. Then perhaps two wrongs can make a right, that is, we combine the two bad diffs into a good diff that is a symmetry of both the metric action and the $\zeta$ action.

Since $\zeta$ measures scalar fluctuations of the expansion of space, we will make the ansatz that the $\operatorname{CDiff(3)}$ diff we look for is a time-dependent dilation of space:

$$
\xi^{\mu}=\left(\frac{c}{\dot{\bar{\phi}}}, c \lambda(t) x^{i}\right) .
$$

Inserting this diff into $\delta \alpha, \delta \beta^{i}$ and $\delta \zeta$, then demanding that the constraint equations be left invariant even at nonzero momentum yields the solution:

$$
\lambda(t)=\lambda_{0}-\int^{t} d t^{\prime}\left(\frac{\dot{\mathrm{H}}}{\dot{\bar{\phi}}}+(\Theta-H) \frac{\ddot{\bar{\phi}}}{\dot{\bar{\phi}}^{2}}\right) .
$$

In what follows next, whenever we mention the shift symmetry acting on $\zeta$, we mean the above diff, not the original diff of the metric action.

One of the crucial aspects of symmetries is that, given one solution to the equations of motion, one can produce new solutions by acting with the symmetry. And if the symmetry is nonlinearly realized, then even the trivial $\zeta=0$ solution can be transformed into 
something nontrivial. Since $\zeta$ measures adiabatic fluctuations of the expansion of space, we call these new solutions adiabatic modes [52]. At first order, the shifty adiabatic mode (SAM) is obtained from the expression of $\delta \zeta$, setting $\zeta=0$ before the transformation:

$$
\zeta_{\text {SAM }}=\mathrm{c}\left(\frac{\mathrm{H}}{\dot{\bar{\phi}}}+\lambda(\mathrm{t})\right) .
$$

Note that, because this gauge transformation is large (it's homogeneous in space, therefore it doesn't vanish at the boundaries of spacetime), it constitutes a genuine physical transformation. Hence, even though $\zeta_{\text {SAM }}$ is a gauge profile, it's a physical mode.

The choice of free parameter $\lambda_{0}$ in (6.78) represents an additional choice of a homogeneous dilation of space. Indeed, once can check that such dilation is immediately a symmetry, in the sense of being a CDiff(3) transformation that preserves the constraints, without any other diff to compensate. The corresponding mode is then the adiabatic dilation mode, or Weinberg mode after its discoverer [52]. In what follows, we will set $\lambda_{0}=0$ but keep in mind the homogeneous dilation exists as a permitted symmetry.

Once we start talking of dilations, one may ask about the rest of the Euclidean conformal group, namely the special conformal transformations (SCT) corresponding to $\delta \vec{x}=x^{2} \vec{b}$. Indeed, it's a symmetry as well, but this one requires a correction to preserve the constraints. The SCT diff vector turns out to be [53]:

$$
\vec{\xi}=2(\vec{b} \cdot \vec{x}) \vec{x}-x^{2} \vec{b}-2 \vec{b} \int^{t} \frac{d t^{\prime}}{\Theta}
$$

\subsubsection{Soft theorems}

We are used to the implication of unbroken symmetries on quantum correlators. The linear realization of a symmetry reduces the amount of information needed to fully described the correlator. For instance, if the vacuum is unbroken under the Euclidean group, then $\langle 0|\phi(\vec{x}) \phi(\vec{y})| 0\rangle$ must be a function of $|\vec{x}-\vec{y}|$. For broken symmetries, the conclusion is more complicated, but arguably more 
interesting as well: it takes the form of a soft theorem, describing the infrared structure of correlators.

To demonstrate the procedure, we will first start with two hard modes (with large momentum) and one soft (with small momentum).

\subsubsection{Two hard modes}

We take as an axiom of our QFT in the FLRW background to be the existence of a momentum space operator product expansion (OPE) $[54,55]$ :

$$
\zeta_{\vec{k}+\vec{q} / 2} \zeta_{-\vec{k}-\vec{q} / 2} \stackrel{\vec{q} \rightarrow 0}{=}(2 \pi)^{3} \delta^{3}(\vec{q}) P(k)+f(k) \zeta_{\vec{q}}+g(k) \dot{\zeta}_{\vec{q}}+\mathcal{O}(q),
$$

where we have assumed Euclidean symmetry is unbroken and taken the operators to be equal time. The first term in the OPE is defined so that, when $\vec{q}=0$ exactly, the ensemble average of the LHS is the power spectrum:

$$
\left\langle\zeta_{\vec{k}} \zeta_{-\vec{k}}\right\rangle=(2 \pi)^{3} \mathrm{P}(\mathrm{k})
$$

Note that in attractor models of inflation, for which the mode $\zeta_{\vec{k}}$ becomes constant the moment $\mathrm{aH}>\mathrm{k}$, are characterized by $\dot{\zeta}$ not being an independent operator in the OPE (6.81) [54]. In our case, we don't want to exclude non-attractor inflation, so we keep $\dot{\zeta}$.

Broken symmetries can be used to fix the functions $f(k)$ and $g(k)$. We use that the action of a conserved charge Q generates the infinitesimal transformation under the symmetry. For instance, consider dilations of space $\delta \vec{x}=-\vec{x}$. This is a large diffeomorphism, so it's a genuine symmetry, and it respects the constraint equations. Let's denote its generator by D. The transformation $\delta \zeta(\vec{x})$ in position space under this diff can be computed as usual, then converted to momentum space:

$$
\delta \zeta_{\vec{k}}=\left[D, \zeta_{\vec{k}}\right]=(2 \pi)^{3} \delta^{3}(\vec{k})-\left(3+\vec{k} \cdot \partial_{\vec{k}}\right) \zeta_{\vec{k}} \cdot
$$

Let us now apply the dilation charge on the OPE. For ease of notation, we call $\vec{k}_{1}=\vec{k}+\vec{q} / 2$ and $\vec{k}_{2}=-\vec{k}-\vec{q} / 2$ 


$$
\begin{aligned}
\left\langle\left[Q, \zeta_{\vec{k}_{1}} \zeta_{\vec{k}_{2}}\right]\right\rangle & =\left[Q, \zeta_{\vec{k}_{1}}\right] \zeta_{k_{2}}+\zeta_{k_{1}}\left[Q, \zeta_{\vec{k}_{2}}\right] \\
& =\left(3+\vec{k}_{1} \cdot \partial_{\vec{k}_{1}}+\vec{k}_{2} \cdot \partial_{\vec{k}_{2}}\right)\left\langle\zeta_{\vec{k}_{1}} \zeta_{\vec{k}_{2}}\right\rangle,
\end{aligned}
$$

where any other contribution drops because $\left\langle\zeta_{\vec{k}}\right\rangle=0$. Since in terms of the power spectrum, $\left\langle\zeta_{\vec{k}_{1}} \zeta_{\vec{k}_{2}}\right\rangle=(2 \pi)^{3} \mathrm{P}\left(\mathrm{k}_{1}\right) \delta^{3}\left(\overrightarrow{\mathrm{k}}_{1}+\overrightarrow{\mathrm{k}}_{2}\right)$, we have that:

$$
\left\langle\left[Q, \zeta_{\vec{k}_{1}} \zeta_{\vec{k}_{2}}\right]\right\rangle=\left(1-n_{s}\right) P\left(k_{1}\right)(2 \pi)^{3} \delta^{3}\left(\vec{k}_{1}+\vec{k}_{2}\right),
$$

where the spectral index $n_{s}$ is simply defined by the above expression.

Meanwhile, acting with D on the RHS merely yields:

$$
\left[f(k)+g(k) \partial_{t}\right]\left\langle\left[Q, \zeta_{\vec{q}}\right]\right\rangle=(2 \pi)^{3} f(k) \delta^{3}(\vec{q}) .
$$

Comparing the LHS to the RHS and recalling $\vec{k}_{1}+\vec{k}_{2}=\vec{q}$, we thus conclude that:

$$
f(k)=\left(1-n_{s}\right) P(k) .
$$

The same argument can be repeated with the diffeomorphism $\mathrm{L}_{\xi}$ corresponding to the shift symmetry. In momentum space, we have:

$$
\delta \zeta_{\vec{k}}=\left[L_{\xi}, \zeta_{\vec{k}}\right]=\lambda(t)\left((2 \pi)^{3} \delta^{3}(\vec{k})-\left(3+\vec{k} \cdot \partial_{\vec{k}}\right) \zeta_{\vec{k}}+\frac{1}{\dot{\bar{\phi}}}\left(H(2 \pi)^{3} \delta^{3}(\vec{k})+\dot{\zeta}_{\vec{k}}\right) .\right.
$$

Again, applying it to the OPE yields:

$$
g(k)=\frac{1}{\Theta} \frac{\dot{\bar{\phi}}}{\ddot{\bar{\phi}}}\left[\left(1-n_{s}\right) P(k) H-\dot{P}(k)\right]
$$


We now wish to take the OPE and add an additional soft mode $\zeta_{\vec{q}}$ on both sides. Actually, there's a slight ambiguity as to whether we add $\zeta_{\vec{q}}$ by left or right multiplication. It turns out that to obtain a well-defined real value for our correlators, we must treat both options the same so that imaginary parts cancel out. We can denote this by anticommutation. For example, in the LHS of the OPE,

$$
\frac{1}{2}\left\{\zeta_{\vec{q}}, \zeta_{\vec{k}-\vec{q} / 2} \zeta_{\vec{k}+\vec{q} / 2}\right\}
$$

accounts for both left and right multiplication. Furthermore, we denote by a prime' the bare correlator:

$$
\left\langle A_{\overrightarrow{k_{1}}} B_{\overrightarrow{k_{2}}} \ldots\right\rangle=(2 \pi)^{3} \delta^{3}(\Sigma \vec{k})\left\langle A_{\overrightarrow{k_{1}}} B_{\overrightarrow{k_{2}}} \ldots\right\rangle^{\prime},
$$

to avoid carrying along factors of $(2 \pi)^{3}$ and momentum-conserving $\delta^{3}(\vec{k})$. So, by attaching the soft mode to our OPE and averaging on both sides, we derive the following soft theorem:

$$
\begin{aligned}
\frac{1}{2}\left\langle\left\{\zeta_{\vec{q}}, \zeta_{\vec{k}-\vec{q} / 2} \zeta_{\vec{I} k+\vec{q} / 2}\right\}\right\rangle^{\prime} & =\mathrm{f}(\mathrm{k})\left\langle\zeta_{\vec{q}} \zeta_{-\vec{q}}\right\rangle^{\prime}+ \\
& \frac{\mathrm{g}(\mathrm{k})}{2}\left\langle\dot{\zeta}_{\vec{q}} \zeta_{-\vec{q}}+\zeta_{\vec{q}} \dot{\zeta}_{-\vec{q}}\right\rangle+\mathcal{O}(\mathrm{q}),
\end{aligned}
$$

which simplifies to:

$$
\begin{aligned}
\left\langle\zeta_{\vec{q}} \zeta_{\vec{k}-\vec{q} / 2} \zeta_{-\vec{k}-\vec{q} / 2}\right\rangle^{\prime}=-\frac{\dot{\bar{\phi}} \dot{P}(q)}{2 \ddot{\bar{\phi}} \Theta}\left[\left(1-n_{s}\right) H P(k)+\dot{P}(k)\right]+ & \left(1-n_{s}\right) P(k) P(q)+\mathcal{O}(q) .
\end{aligned}
$$

Actually, an additional large diff that also satisfies the constraint equations is the special conformal transformation $\delta \vec{x}=-|x|^{2} \vec{u}$ along a unit vector $\vec{u}$. Repeating the entire argument with this symmetry then sets that the first correction to the above relation starts at $\mathcal{O}\left(q^{2} / k^{2}\right)$ rather than $\mathcal{O}(q / k)[54,56]$. This is called an enhanced soft limit, a telltale sign there are multiple broken symmetries. 
Note that these relations are model-dependent. The function $\Theta$ is simply $\mathrm{H}$ if the Lagrangian of the scalar field contains only first derivatives, but can be more complicated otherwise. Similarly, the various appearances of $\bar{\phi}$ require knowledge of the scalar background. The spectral index $n_{s}$ and the power spectrum $P(k)$ can be directly measured from the present-day $\mathrm{CMB}$ just fine, but the time derivative $\dot{P}(k)$ would require us to observe the $\mathrm{CMB}$ at different points in time, which is incompatible with how observational cosmology is performed.

As we said before, a certain collection of models dubbed attractor models display the feature that $\zeta_{\vec{k}}$ freezes outside the horizon, that is, $\dot{\zeta}_{\vec{k}}=0$ the moment that aH $>\mathrm{k}$. Thus the mode ceases to evolve during inflation. ${ }^{3}$ Assuming we evaluate the soft theorem at the moment when the hard modes freeze, then $\dot{P}(k)=0$, and the result simplifies:

$$
\left\langle\zeta_{\vec{q}} \zeta_{\vec{k}-\vec{q} / 2} \zeta_{-\vec{k}-\vec{q} / 2}\right\rangle^{\prime}=\left(1-n_{s}\right) P(k) P(q)+\mathcal{O}\left(q^{2}\right) .
$$

This theorem is now model-independent (other than the mild assumption of the attractor) and can be directly tested with CMB information. It is the famous Maldacena consistency relation for single field inflation. It is often proclaimed that observing its violation would rule out all models of single field inflation, though in reality only attractor models can be falsified in this manner.

\subsubsection{Multiple hard modes}

The technique follows identically even if we wish to consider correlators of $n$ hard modes, that is, modes with large momentum. The n-hard OPE now takes the form [57]:

$$
\prod_{a=1}^{n} \zeta_{\vec{k}_{a}-\vec{q} / n} \stackrel{\vec{q} \rightarrow 0}{=}(2 \pi)^{3} B_{n}\left(\vec{k}_{a}\right) \delta^{3}(\vec{q})+f_{n}\left(\vec{k}_{a}\right) \zeta_{-\vec{q}}+g_{n}\left(\vec{k}_{a}\right) \dot{\zeta}_{-\vec{q}}+\mathcal{O}(q) .
$$

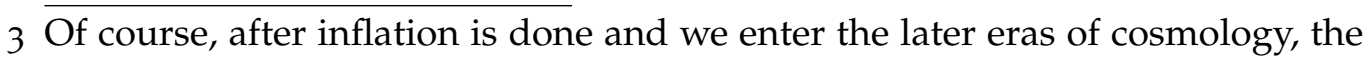
mode unfreezes as the horizon grows. But then we are in the realm of well understood physics, so we know how everything should evolve. 
Again, the first term in the OPE is fixed by the definition of the polyspectrum:

$$
\left\langle\prod_{a=1}^{n} \zeta_{\vec{k}_{a}}\right\rangle \stackrel{\text { def }}{=}(2 \pi)^{3} B_{n}\left(\vec{k}_{a}\right) \delta^{3}\left(\Sigma \vec{k}_{a}\right) .
$$

As illustrated in the previous section, applying the dilation and shift symmetries let us fix the $f_{n}$ and $g_{n}$ functions, such that:

$$
\begin{aligned}
f_{n}\left(\vec{k}_{a}\right) & =-\mathcal{D}_{(\mathfrak{n})} B_{n}\left(\vec{k}_{a}\right), \\
g_{n}\left(\vec{k}_{a}\right) & \left.=-\frac{\dot{\bar{\phi}}}{\ddot{\bar{\phi}} \Theta}\left[-H \mathcal{D}_{(} n\right) B_{n}\left(\vec{k}_{a}\right)-\dot{B}_{\mathfrak{n}}\left(\vec{k}_{a}\right)\right], \\
\mathcal{D}_{(n)} & \stackrel{\text { def }}{=}\left[3(n-1)+\sum_{a=1}^{n} \vec{k}_{a} \cdot \partial_{\vec{k}_{a}}\right] .
\end{aligned}
$$

The n-hard soft theorem then is:

$$
\begin{aligned}
\frac{1}{2}\left\langle\left\{\zeta_{\vec{q},} \prod_{a=1}^{n} \zeta_{\vec{k}_{a}-\vec{q} / n}\right\}\right\rangle^{\prime}= & f_{n}\left\langle\zeta_{\vec{q}} \zeta_{-\vec{q}}\right\rangle^{\prime} \\
& +\frac{1}{2} g_{n}\left\langle\dot{\zeta}_{\vec{q}} \zeta_{-\vec{q}}+\zeta_{\vec{q}} \dot{\zeta}_{-\vec{q}}\right\rangle^{\prime}, \quad(6.101) \\
\Longrightarrow\left\langle\zeta_{\vec{q}} \prod_{a=1}^{n} \zeta_{\vec{k}_{a}-\vec{q} / n}\right\rangle^{\prime}= & -\frac{\dot{\bar{\phi}} \dot{P}(q)}{2 \ddot{\vec{\phi}} \Theta}\left[H \mathcal{D}_{(\mathfrak{n})} B_{\mathfrak{n}}\left(\vec{k}_{\mathfrak{a}}\right)+\dot{B}_{\mathfrak{n}}(\vec{k})\right] \\
& -P(q) \mathcal{D}_{(\mathfrak{n})} B_{\mathfrak{n}}\left(\vec{k}_{\mathfrak{a}}\right)+\mathcal{O}(\mathbf{q}) .
\end{aligned}
$$

Again, usage of the special conformal symmetry enhances this soft theorem so that the corrections actually start at $\mathcal{O}\left(\mathrm{q}^{2}\right)$.

\subsection{SOME PHENOMENOLOGY FOR $\mathcal{L}\left(\mathrm{g}^{00}\right)$}

We will now discuss some phenomenology for the EFT of inflation. For the sake of expediency, we will disregard extrinsic curvature 
terms and work with only $\delta g^{00}$ At the level of the scalar field action, this corresponds to a shift-symmetric theory without higher derivatives, namely:

$$
S=\int d^{4} x \sqrt{-g} P(X), \quad X=-\frac{1}{2} g^{\mu v} \partial_{\mu} \phi \partial_{\nu} \phi
$$

\subsubsection{Ultra slow roll inflation}

First, let's examine the background inflationary dynamics. We have already seen the various FLRW parameters in the previous sections, but let's discuss them more explicitly. Recall that given the FLRW scale factor $a$ and Hubble parameter $H=\partial_{t} \log a$, we define the first two slow roll parameters as:

$$
\varepsilon \stackrel{\text { def }}{=}-\frac{\dot{H}}{\mathrm{H}^{2}}, \quad \eta \stackrel{\text { def }}{=} \frac{\dot{\varepsilon}}{\varepsilon \mathrm{H}} .
$$

Slow roll (SR) inflation is defined by a near de Sitter background, meaning $\mathrm{H}$ nearly constant so $\varepsilon \ll 1$, and that evolves slowly in time, so $|\eta| \ll 1$. Ultra slow roll inflation (USR), on the other hand, is a a near de Sitter state that is allowed to evolve quickly, that is, $\eta$ can be large [58]. Typically the system evolves towards de Sitter, i. e., pushing $\varepsilon$ to zero, so that $\eta<0.4$

It turns a shift-symmetric EFT without extrinsic curvature terms supports only USR, not SR. Recall the recursive relation:

$$
\dot{\mathrm{d}}_{\mathrm{n}}=2 \mathrm{H}(3-2 \varepsilon+\eta)\left(n \mathrm{~d}_{\mathrm{n}}-\mathrm{d}_{\mathrm{n}+1}\right),
$$

as well as the speed of sound:

$$
\frac{1}{c_{s}^{2}}=1+\frac{2 \mathrm{~d}_{2}}{\varepsilon M_{P 1} \mathrm{H}^{2}} .
$$

4 The names for these conditions make more sense when working with the scalar field Lagrangian, in which case they follow from the nature of the field's evolution through a potential. In the spirit of the EFT, we wish to avoid using the more fundamental scalar. 
We can now rearrange the $d=1$ relation as:

$$
\varepsilon\left(3+3 c_{s}^{2}-2 \varepsilon+\eta\right)=0 .
$$

Imposing the slow roll conditions $\varepsilon \approx 0$ and $\eta \approx 0$, the above can only be true if $c_{s}^{2} \approx-1$-indicating the background isn't stableor $\varepsilon=0$ exactly, which corresponds to models of ghost inflation [59]. Thus, in general, we expect only ultra slow roll in this case, corresponding to $\varepsilon \approx 0$ and $\eta \approx-3\left(1+\mathrm{c}_{s}^{2}\right)$.

Thus the argument that an approximate shift symmetry leads to the near constancy of the EFT parameters in slow roll inflation is a bit dubious: shift symmetry and slow roll are incompatible! Indeed, armed with (6.107), we can now write the recursive relation in the more compact form:

$$
\dot{\mathrm{d}}_{\mathrm{n}}=2 \mathrm{Hc}_{\mathrm{s}}^{2}\left(\mathrm{nd}_{\mathrm{n}}-\mathrm{d}_{\mathrm{n}+1}\right) .
$$

Unless we are in Minkowski space, the coefficients can only be constant if $c_{s}^{2}=0$ which is well outside the EFT's domain of validity.

So far we have worked only at the level of the EFT, utilizing only our recursive relations imposed by the broken shift symmetry. For completeness's sake, one may wonder how this argument works at the level of the more fundamental scalar field. Given the $P(X)$ action in (6.103), its background stress-energy is that of a perfect fluid:

$$
\begin{gathered}
\overline{\mathrm{T}}_{\mu \nu}=\frac{-2}{\sqrt{-g}} \frac{\delta S}{\delta g^{\mu \nu}}=(\rho+p) n_{\mu} n_{v}+p g_{\mu \nu} \\
\rho=-P+2 X P_{, X}, \quad p=P, \quad u_{\mu}=\delta_{\mu}^{0} .
\end{gathered}
$$

The first two Friedmann equations depend on the density $\rho$ and pressure $\mathrm{p}$; they can be used to solve $\mathrm{P}$ and $\mathrm{P}_{X}$ in terms of the FLRW parameters $\mathrm{H}$ and $\varepsilon$. The speed of sound is defined by:

$$
c_{s}^{2} \stackrel{\text { def }}{=} \frac{\partial p}{\partial \rho}=\frac{p, x}{\rho, x},
$$


which let's us recast $P, x x$. Lastly, a time derivative of either Friedmann equation lets us replace $\dot{X}$ in terms of the other FLRW background parameters at the cost of introducing $\eta$. All put together, we have:

$$
\begin{aligned}
\mathrm{P} & =M_{\mathrm{Pl}}^{2} \mathrm{H}^{2}(2 \varepsilon-3) \\
X \mathrm{P}, \mathrm{X} & =M_{\mathrm{Pl}}^{2} \mathrm{H}^{2} \varepsilon \\
\mathrm{X}^{2} \mathrm{P}, \mathrm{XX} & =M_{\mathrm{Pl}}^{2} \mathrm{H}^{2}\left(\frac{1-\mathrm{c}_{s}^{2}}{2 \mathrm{c}_{s}^{2}}\right) \varepsilon \\
\frac{\dot{X}}{\mathrm{HX}} & =\frac{6 \mathrm{c}_{s}^{2}(\eta-2 \varepsilon+2)}{1+3 \mathrm{c}_{\mathrm{s}}^{2}} .
\end{aligned}
$$

Now it's time to compute the equation of motion for the scalar background $\bar{\phi}(\mathrm{t})$ and unpack it in terms of the above:

$$
\begin{aligned}
\frac{d}{d t}\left[a^{3} \sqrt{X} P, X\right] & =0 \\
\Longrightarrow \varepsilon\left(3+3 c_{s}^{2}-2 \varepsilon+\eta\right) & =0 .
\end{aligned}
$$

\subsubsection{Consistency relation}

The soft theorem for two hard modes serves as consistency relation between the power spectrum (which we have already measured) and the bispectrum (which we hope to measure in the future). Recall that our soft theorem (6.123) depended on $\dot{\bar{\phi}} / \ddot{\bar{\phi}}$ as well as $\Theta$. The former we can solve in terms of the background (6.53), while the latter is just $\mathrm{H}$ when the action is devoid of extrinsic curvature terms. For simplicity of computation, we will focus on the canonical USR case, $P(X)=X+V_{0}$, which leads to $c_{s}^{2}=1$, though the argument can be generalized to more complicated actions.

Following the usual mode function methods for computing the $\zeta$ correlators leads to: 


$$
\begin{aligned}
\left\langle\zeta_{\vec{k}} \zeta_{-\vec{k}}\right\rangle^{\prime} & =\frac{\mathrm{H}^{2}}{4 \mathrm{M}_{\mathrm{Pl}}^{2} \varepsilon} \times \frac{1}{\mathrm{k}^{3}} \\
& =\mathrm{P}(\mathrm{k}), \\
\left\langle\zeta_{\vec{k}_{1}} \zeta_{\vec{k}_{2}} \zeta_{\vec{k}_{3}}\right\rangle & =\frac{3 \mathrm{H}^{4}}{16 \mathrm{M}_{\mathrm{Pl}}^{4} \varepsilon^{2}} \times \frac{\Sigma_{i} k_{i}^{3}}{\Pi_{i} k_{i}^{3}} \\
& =3 \sum_{i>j} \mathrm{P}\left(\mathrm{k}_{i}\right) \mathrm{P}\left(\mathrm{k}_{\mathrm{j}}\right) .
\end{aligned}
$$

Writing $\vec{k}_{1}=\vec{q}, \vec{k}_{2}=\vec{k}-\vec{q} / 2$ and $-\vec{k}-\vec{q} / 2$, the squeezed bispectrum is the soft limit $\vec{q} \rightarrow 0$ :

$$
\left\langle\zeta_{\vec{q}} \zeta_{\vec{k}-\vec{q} / 2} \zeta_{-\vec{k}-\vec{q} / 2}\right\rangle=6 P(q) P(k)+\mathcal{O}(q),
$$

using the permutation symmetry $\vec{k}_{1} \approx-\vec{k}_{2}$ in the sum. This is a direct derivation of the consistency relation between squeezed bispectrum and power spectrum by explicitly computing the correlators [6o].

Let's check the same result at the level of the soft theorem. We still compute the power spectrum as above, but refrain from computing the bispectrum. Using the scalar's equation of motion, $\ddot{\bar{\phi}}+3 \mathrm{H} \dot{\bar{\phi}}=0$, together with the Friedmann equations, one finds $\varepsilon \sim a^{-6}$, so $\dot{\varepsilon}=-6 \mathrm{H} \varepsilon$. Since $\mathrm{P}(\mathrm{k}) \sim \varepsilon^{-1}$ it follows $\dot{\mathrm{P}}(\mathrm{k}) \approx 6 \mathrm{HP}(\mathrm{k})$. Furthermore, the power spectrum satisfies $P(k) \sim k^{-3}$, hence scale invariant: $n_{s}=0$. Inserting these facts about $\dot{P}$ and $n_{s}$ into the soft theorem we derived from symmetry principles,

$$
\begin{aligned}
\left\langle\zeta_{\vec{q}} \zeta_{\vec{k}-\vec{q} / 2} \zeta_{-\vec{k}-\vec{q} / 2}\right\rangle^{\prime} \approx-\frac{\dot{\bar{\phi}} \dot{P}(q)}{2 \ddot{\phi} \Theta}\left[\left(1-n_{s}\right) H P(k)+\dot{P}(k)\right] \\
+\left(1-n_{s}\right) P(k) P(q),
\end{aligned}
$$

together with $\Theta=\mathrm{H}$ for EFTs without higher derivatives, yields the consistency relation we derived explicitly. 


\subsubsection{Non-Gaussianities}

We will now discuss interactions in the pure $\delta g^{00}$. In particular, we're interested in contributions to the non-Gaussian parameter $f_{\mathrm{NL}}$ of primordial fluctuations. Without the higher derivative terms included in the extrinsic curvature $\delta \mathrm{K}_{\mu v}$, all coefficients in the EFT are uniquely determined in terms of the background evolution and associated derivatives. The interpretation of this is that the fluctuations are adiabatic: each region of space is evolving exactly like the background, it's just that some regions are lagging behind and others blazing ahead in this evolution.

We want to study interactions in the shift-symmetric EFT and check if any interaction term normally neglected in the usual slow roll analysis might be relevant here. Let's write the action for $\delta g^{00}$ up to cubic order. Remember, $d_{0}, d_{1}$ and $d_{2}$ are fixed by the two Friedmann equations and the speed of sound definition, respectively. For the cubic interactions, we need $d_{3}$, found via the $n=2$ relation:

$$
\dot{\mathrm{d}}_{2}=2 \mathrm{Hc}_{\mathrm{s}}^{2}\left(2 \mathrm{~d}_{2}-\mathrm{d}_{3}\right) \text {; }
$$

the price we pay is the introduction of the sound speed rate of change, $s \stackrel{\text { def }}{=} \dot{\mathrm{c}}_{\mathrm{s}} / \mathrm{c}_{\mathrm{s}} \mathrm{H}$. We now unpack the Lagrangian up to $\left(\delta \mathrm{g}^{00}\right)^{3}$ :

$$
\begin{gathered}
\mathcal{L}=-M_{P l}^{2}\left(3 H^{2}+2 \dot{H}\right)+M_{P l}^{2} \dot{H} \delta g^{00}+\frac{M_{P l}^{2} \dot{H}}{4} \frac{c_{s}^{2}-1}{c_{s}^{2}}\left(\delta g^{00}\right)^{2} \\
+\frac{M_{P l}^{2} \dot{H}}{6 c_{s}^{2}}\left[c_{s}^{2}-1+\frac{1}{3 c_{s}^{2}} \frac{2 s+\left(1-c_{s}^{2}\right)(2 \varepsilon-\eta)}{4}\right]\left(\delta g^{00}\right)^{3} .
\end{gathered}
$$

In the literature (e.g., $[19,61])$, it's customary to describe the cubic interaction in terms of a parameter $\tilde{c}_{3}$. For the reader more accustomed with this notation, we provide a conversion between $\tilde{c}_{3}$ and the parameter $d_{3}$ in our EFT. By direct comparison, we see:

$$
\tilde{\mathrm{c}}_{3}\left(\mathrm{c}_{\mathrm{s}}^{-2}-1\right)=\frac{1}{2}\left(3 \mathrm{c}_{\mathrm{s}}^{2}-4+\mathrm{c}_{\mathrm{s}}^{-2}\right)+\frac{\mathrm{s}}{3 \mathrm{c}_{\mathrm{s}}^{2}}
$$


As we discussed before, for low energy phenomenology, it is more transparent to work with the Stückelberg scalar perturbation $\pi$ rather than the metric perturbation $\delta g^{00}$ :

$$
\delta g^{00}=g^{00}+1 \rightarrow g^{\mu v} \partial_{\mu} \pi \partial_{n} u \pi+1 .
$$

After some computation, we see there are no terms linear in $\pi$ (so $\pi$ describes fluctuations around the background, but doesn't govern the background's dynamics), while its quadratic action is:

$$
S_{\pi}^{(2)}=\int d^{4} x \sqrt{-g} \frac{\varepsilon M_{P 1}^{2} H^{2}}{c_{s}^{2}}\left[\dot{\pi}^{2}-c_{s}^{2} \frac{\left(\partial_{i} \pi\right)^{2}}{a^{2}}-3 c_{s}^{2} H^{2} \varepsilon \pi^{2}\right] .
$$

For ease of notation when we compute the cubic action, it's convenient to make a rescaling of space $x^{i} \rightarrow x^{i} / c_{s}$, which might be time-dependent if $c_{s}$ is. This is merely to render the expressions more compact by writing it as if Lorentz invariance were a symmetry. Furthermore, we wish to canonically normalize the field so it's a proper scalar of mass dimension 1 . So perform the redefinitions:

$$
\begin{aligned}
& \tilde{\chi}^{i} \stackrel{\text { def }}{=} \frac{1}{c_{s}} x^{i} \\
& \pi_{c} \stackrel{\text { def }}{=} \sqrt{2 c_{s} M_{P l}^{2} H^{2} \varepsilon} \pi \stackrel{\text { def }}{=} f_{\pi}^{2} \pi,
\end{aligned}
$$

from which it follows that

$$
S_{\pi_{c}}^{(2)}=\frac{1}{2} \int d t d^{3} \tilde{x}\left[\left(\tilde{\partial}_{\mu} \pi_{c}\right)^{2}-m^{2} \pi_{c}^{2}\right],
$$

with the time-dependent mass

$$
\begin{aligned}
m^{2}=\frac{H^{2}}{4}\left[-5 s^{2}+8 \varepsilon^{2}+\right. & s(6+6 \varepsilon-4 \eta)+\eta(6+\eta) \\
& \left.-2 \varepsilon\left(6+6 c_{s}^{2}+5 \eta\right)+2 \frac{\dot{s}+\dot{\eta}}{H}\right] .
\end{aligned}
$$


Using this notation, the cubic action can now be computed in a slightly more convenient way. It reads:

$$
\begin{aligned}
& S_{\pi_{\mathrm{c}}}^{(3)}=\int \mathrm{dt} \mathrm{d}^{3} \tilde{x}\left[\frac{\mathrm{c}_{s}^{2}-1}{2 \Lambda_{\star}^{2}} \dot{\pi}_{\mathrm{c}} \frac{\left(\tilde{\partial}_{i} \pi_{\mathrm{c}}\right)^{2}}{\mathrm{a}^{2}}+\frac{\left(1-\mathrm{c}_{s}^{2}\right)\left(2 \tilde{\mathrm{c}}_{3}+3 \mathrm{c}_{s}^{2}\right)}{6 \Lambda_{\star}^{2}} \dot{\pi}_{\mathrm{c}}^{3}\right. \\
& +\frac{\left(1-\mathrm{c}_{s}^{2}\right) s-\left(1-3 \mathrm{c}_{s}^{2}\right)(2 \varepsilon-\eta)}{4 \Lambda_{\star}^{2}} \mathrm{H} \pi_{\mathrm{c}} \frac{\left(\tilde{\partial}_{i} \pi_{\mathrm{c}}\right)^{2}}{\mathrm{a}^{2}} \\
& +\frac{\mathrm{c}_{s}^{2}\left[\left(3 \mathrm{c}_{s}^{2}-7\right) s+\left(1-3 \mathrm{c}_{s}^{2}\right)(2 \varepsilon-\eta)\right]-2 \tilde{\mathrm{c}}_{3}\left(1-\mathrm{c}_{s}^{2}\right)(\mathrm{s}-2 \varepsilon+\eta)}{4 \Lambda_{\star}^{2}} \mathrm{H} \pi_{\mathrm{c}} \dot{\pi}_{\mathrm{c}}^{2} \\
& \left.+\frac{\mathrm{H}^{3}}{\Lambda_{\star}^{2}} \mathrm{C}_{030} \pi_{\mathrm{c}}^{3}\right] .
\end{aligned}
$$

Here, we have defined the scale $\Lambda_{\star}$ :

$$
\Lambda_{\star}=\mathrm{c}_{\mathrm{s}} \mathrm{f}_{\pi}
$$

which acts as a coupling constant. For the EFT to be valid, weak coupling must apply, hence $\Lambda_{\text {star }} \gg \mathrm{H}$. The coefficient $\mathrm{C}_{030}$ is a complicated combination of slow roll parameters; we omit it for expediency since we won't study this interaction term.

We want to focus on the $\pi_{\mathrm{c}}\left(\partial \pi_{\mathrm{i}} \pi_{\mathrm{c}}\right)^{2}$ operator. The reason is that an approximate shift symmetry is often involved in discussions of slow roll inflation to suppress any operator containing $\pi_{\mathfrak{c}}$ without derivatives. But here we have precisely the consequences of a shift symmetry on the EFT coefficients, and it's not true in general that it is suppressed. For example, take its contribution to the nonGaussian parameter $f_{N L}$ and compare it against that of $\dot{\pi}_{\mathrm{c}}\left(\partial \pi_{\mathrm{i}} \pi_{\mathrm{c}}\right)^{2}$; we can estimate this ratio as:

$$
\frac{f_{N L}^{\dot{\tau}_{\mathrm{c}}\left(\tilde{\partial}_{i} \pi_{c}\right)^{2}}}{f_{\mathrm{NL}}^{\pi_{\mathrm{c}}\left(\tilde{\partial}_{i} \pi_{\mathrm{c}}\right)^{2}}} \sim \frac{1-c_{s}^{2}}{\left(1-\mathrm{c}_{\mathrm{s}}^{2}\right) s-\left(1-3 \mathrm{c}_{\mathrm{s}}^{2}\right)(2 \varepsilon-\eta)} .
$$

The denominator is not suppressed in general for a shift-symmetric theory, since as we saw in (6.107) some strong time dependence in the necessary to solve the background equations of motion. This leads to $\eta \sim 1$ and possibly $s \sim 1$ as well. 


\subsubsection{Cutoff scale}

In the previous section, we saw how in an exactly shift-symmetric model of inflation, some contributions to $f_{\mathrm{NL}}$ normally taken to be negligible in slow roll inflation might not be so. Can similar arguments lead to a modification of the EFT strong coupling scale? That's the energy at which one or more operators are no longer perturbatively unitary, indicating the breakdown of the EFT and the requirement for a new theory to UV-complete it. Let's perform the same analysis as in [61], but now for our EFT. From dimensional analysis, any operator in the EFT can denoted as:

$$
C_{n m k} \frac{H^{m}}{\Lambda_{\star}^{2 n-4}} \times \pi_{c}^{m} \dot{\pi}_{c}^{n-m-2 k}\left(\tilde{\partial}_{i} \pi_{c}\right)^{2 k},
$$

where $C_{n m k}$ is a dimensionless coefficient depending on the slow roll parameters. Now, if we take the usual approach of the EFT of inflation where $\pi_{\mathrm{c}}$ 's without derivatives are ignored, then essentially $m=0$ and the only scale is $\Lambda_{\star}$. In our case, though, we are permitting $m \neq 0$. Because of this, there's a collection of energy scales associated with the operator above:

$$
E_{n m k}=\frac{1}{C_{n m k}}\left(\frac{\Lambda_{\star}}{H}\right)^{\frac{m}{2 n-m-4}} \times \Lambda \star .
$$

Now, $E_{n m k}$ typically increases with $m$, which is good, as these higher energy scales are above the cutoff we already have for our theory. There is one exception, of course, when $2 n-m-4<0$. But the only such operator is the cubic $\pi_{c}^{3}$ for which $n=m=3$ and $k=0$. But this operator isn't important for our discussion here. So indeed there's no modification in the discussion of the cutoff scale: it remains $\Lambda_{\star}$, as in the standard slow roll EFT, assuming all the $\mathrm{C}_{n \mathrm{nk}}$ are of the same order of magnitude.

For the cubic terms, we can be more explicit about the form of the $\mathrm{C}_{\mathrm{nmk}}$. From [61], we have the scales associated with the following two operators:

$$
\Lambda_{\dot{\pi}_{\mathrm{c}}\left(\tilde{\partial}_{i} \pi_{\mathrm{c}}\right)^{2}} \sim \frac{\Lambda_{\star}}{\left(1-\mathrm{c}_{\mathrm{s}}^{2}\right)^{1 / 2}}, \quad \Lambda_{\dot{\pi}_{\mathrm{c}}^{3}} \sim \frac{\Lambda_{\star}}{\left(1-\mathrm{c}_{\mathrm{s}}^{2}\right)^{1 / 2}\left(\mathrm{c}_{\mathrm{s}}^{2}+3 \tilde{\mathrm{c}}_{3} / 3\right)^{1 / 2}}
$$


$(6.138)$

to which we add the scale of the $\pi_{\mathfrak{c}}\left(\tilde{\partial}_{\mathfrak{i}} \pi_{\mathfrak{c}}\right)^{2}$ calculated in the same manner:

$$
\Lambda_{\pi_{\mathrm{c}}\left(\tilde{\partial}_{i} \pi_{\mathrm{c}}\right)^{2}} \sim \frac{\Lambda_{\star}}{\left(1-\mathrm{c}_{s}^{2}\right) s-\left(1-3 \mathrm{c}_{s}^{2}\right)(2 \varepsilon-\eta)} \frac{\Lambda_{\star}}{\mathrm{H}}
$$

Indeed, in slow roll with $\varepsilon, \eta, s \ll 1$, it's true the scale (6.139) will be magnified compared to (6.138) and thus doesn't modify the overall cutoff scale of the theory; strong coupling is still determined by (6.138). But for exact shift symmetry, $\eta$ and possibly $s$ are of order unity, so the prefactor isn't necessarily greatly enhanced. That being said, it is still true that $\Lambda_{\star}>H$, so again this scale might get pushed upwards.

Nonetheless, notice that if $c_{s} \approx 1$ can greatly magnify the scales (6.138). So it seems these scales might get pushed above (6.139), in particular if $c_{s}$ is sufficiently close to unity:

$$
\sqrt{1-c_{s}^{2}}>\frac{H}{\Lambda_{\star}}
$$

This means the cutoff will then be given by the lower scale (6.139), a change from the standard EFT. This is quite significant, as it represents the energy at which new physics joins the game.

If this happens we say the theory is fine-tuned, as from dimensional analysis grounds an operator such as $\pi_{\mathfrak{c}}\left(\tilde{\partial}_{\mathfrak{i}} \pi_{\mathfrak{c}}\right)^{2}$ cannot be strongly coupled at scales lower than one with more derivatives like $\dot{\pi}_{\mathrm{c}}\left(\tilde{\partial}_{\mathrm{c}}\right)^{2}$ and $\dot{\pi}_{\mathrm{c}}^{3}$. While this isn't required, if one wishes the EFT to be technically natural, then its cutoff scale can't be changed in this way. Alternatively, if the UV scale is observed to be lower than expected, the naturalness principle has failed for this theory. 


\subsection{DISCUSSION}

In this chapter we discussed the theory of shift-symmetric cosmologies. It unifies two concepts: the EFT of cosmology (or, more specifically, of inflation) built out of broken diffeomorphism together with a scalar field displaying an internal shit symmetry invoked to deal with UV considerations of the inflaton potential.

The primary product of this study is the constraint on the EFT of inflation parameters. In the presence of the shift symmetry, all coefficients cascade into each other through nontrivial recurrence relations that are background-dependent. Hence, the shift symmetry drastically increases the predictive power of the EFT by eliminating a great number of free parameters.

We also examined the infrared structure of a shift-symmetric cosmology, which is affected by its broken symmetries, large diffs and shifts included. From this we were able to derive the shiftsymmetric adiabatic, a seemingly trivial solution (by virtue of looking like a gauge profile) but that is a genuinely physical mode, then used it to derive soft theorems for the correlation functions. These constraints can be directly through observations of the primordial bispectrum (or higher polyspectra) in the squeezed limit.

Finally, we studied some phenomenology of ultra slow inflation, a subtype of shift-symmetric inflation, for the purposes of displaying how to employ these tools in a more concrete manner.

A natural extension of this work would be to consider more complex symmetry breaking patterns for inflation other than just the simple shift. As discuss in the opening Section 6.2, in flat spacetime the bulk (i. e., gravitational) properties of a condensed matter system can be classified in terms of its mixed spacetime-internal symmetry breaking pattern. The same idea applied to curved spacetime would let us classify different kinds of inflationary substances, and derive the associated EFTs.

Another important continuation is the issue of approximate symmetries being broken. In reality, inflation displays merely an approximate shift symmetry, so we would still need to derive the precise effects that this small explicit symmetry breaking introduces in all the results of this chapter. 



\subsection{INTRODUCTION}

Broken spacetime symmetries violate Goldstone's theorem in that the Goldstone operators don't necessarily have to vanish at zero momentum -in other words, spacetime Goldstones can appear completely without derivatives in the action. That is already peculiar, but much more mysterious is the theorem's failure to even count the number of Goldstone bosons. No longer are they in an one-to-one correspondence with the number of broken generators; rather, some bosons might end up inessential and simply don't appear in the theory.

Typically in the literature, such a phenomenon is presented in terms of the so-called inverse Higgs constrains (IHC) [62, 63]. The Maurer-Cartan procedure for the coset construction can produce invariant or covariant objects in which one of the bosons appears algebraically while the other appears with derivatives. Setting such object to zero then let us solve for the former in terms of the latter. This is often reframed in terms of equations of motion: the inessential Goldstone has a mass that is parametrically higher than the essential's, and thus gets integrated out at low energies [48, 64]. Solving its equation of motion and inserting the solution back into the action is precisely equivalent to imposing the IHC. However, as we will see in Section 7.3.1, this isn't the full story: some IHCs apply even to massless fields!

In the normalization procedure, this phenomenon manifests as hierarchies in the transformation rules. We define an inessential Goldstone boson in the following way. Suppose we have the spacetime coordinates $x$ together with two kinds of bosons, $\pi$ and $\xi$. They might be scalars, vectors or tensors; we're suppressing indices for simplicity. Then $\pi$ is essential and $\xi$ inessential if the following two conditions hold: 
1. The $x$ and $\pi$ are in a multiplet of $G$, in the sense they transform (nonlinearly) among themselves, without reference to $\xi$.

2. The group action on $x$ and $\pi$ is faithful. This means the two fully realize the group: their transformations depend on all group parameters.

Schematically, we can denote these properties in the following way. If we write as $g_{a}$ the following group parameters contained in a group element $g \in G$, then the transformations of $x, \pi$ and $\xi$ must take the following form:

$$
\begin{aligned}
& x \rightarrow \tilde{x}(x, \pi ; g), \\
& \pi \rightarrow \tilde{\pi}(x, \pi ; g), \\
& \xi \rightarrow \tilde{\xi}(x, \pi, \xi ; g), \\
& \frac{\partial \tilde{x}}{\partial g_{a}} \neq 0 \text { or } \frac{\partial \tilde{\pi}}{\partial g_{a}} \neq 0,
\end{aligned}
$$

for all a (as is standard, the "or" here is inclusive).

If these conditions are satisfied, then we can simply discard $\xi$ and forget it exists. The normalization procedure will still be consistent (because of property 1) and we will still be able to fix all group parameters through normalizations (because of property 2).

Studying these kinds of hierarchies that can arise in the coset construction is important if one wishes to understand questions of uniqueness and classification of EFTs. In particular, we must determine if a certain hierarchy between Goldstone bosons is a property intrinsic to the symmetry breaking pattern itself or if it's merely a product of the way we chose to order the coset space lift.

\section{Outline}

This chapter is organized as follows:

- In Section 7.2, we discuss the issue of ordering the group elements in the coset space parametrization and whether or not it can affect the result. We show that for nonsimple groups, 
preferred orderings are given by the Levi decomposition and use this idea to derive two notions of planar geometry.

- In Section 7.3, we study the hierarchies of Goldstone bosons in flat spacetimes for two kinds of theories involving inessential vectors (breaking the conformal group to Poincaré) and tensors (breaking the special Galileo group to Poincaré). We apply the techniques for coset ordering we discussed in the previous section to complete the analysis.

- In Section 7.4, we extend the discussion of Goldstone hierarchies to curved spacetimes, constructing an explicit example living in de Sitter spacetime and containing both a scalar and a vector with neither being inessential.

\subsection{COSET ORDERING}

When all Goldstones are essential, there can be no question that any parametrization of the coset lift is equivalent. After all, by the orbit-stabilizer theorem, all homogeneous spaces representing the same coset space are isomorphic to each other, and so the Smatrix will be automatically unchanged under these isomorphisms. Indeed, for internal symmetries where all Goldstones are essential, it is known that the coset construction is unique [63].

For inessential Goldstones, the situation is more complicated, because there's an additional structure ascribed to the coset space, that of the Goldstone hierarchy. The orbit-stabilizer theorem only guarantees the isomorphism is a group homomorphism, that is, that it preserves the group product. It says nothing about additional structure such as topology or hierarchy. We therefore aren't immediately capable of deducing the S-matrix is always invariant under changes of the coset coordinates.

To understand this issue properly, we must know how to order the group elements properly in order to reveal the different hierarchies that can be induced on the elements of the coset construction. For a nonsimple group, at least two preferred orderings arise, based on the Levi decomposition discussed in Section 2.2. 


\subsubsection{Levi orderings}

Suppose we wish to parametrize the coset space $G / U$, where $G$ is nonsimple but $\mathrm{U}$ is simple or semisimple. Denote by $\mathrm{P}$ the generators in the radical of $G$ and by $T$ those of the simple factor. Furthermore, if a $\mathrm{T}$ is broken (i. e., $\mathrm{T} \notin \mathrm{S}$ ) denote it by $\mathrm{A}$; conversely, denote it by $\mathrm{V}$ if it's unbroken (i.e., $\mathrm{T} \in \mathrm{S}$ ). Then there are two orderings that follow naturally from the Levi decomposition:

$$
\begin{array}{ll}
\ell_{R} \stackrel{\text { def }}{=} e^{z P} e^{\xi A} & (z \text { transforms by itself }), \\
\ell_{L} \stackrel{\text { def }}{=} e^{\xi, A} e^{z P} & (\xi \text { transforms by itself }) .
\end{array}
$$

To show this, we require the following braiding identities:

$$
\begin{aligned}
& e^{a^{\mathrm{PP}} e^{u T}}=e^{u T} e^{M_{u} \mathrm{aP}}, \\
& e^{a \mathrm{P}} e^{\nu V}=e^{\nu V} e^{M_{\nu} \mathrm{aP}}, \\
& e^{\mathrm{aP}} e^{\xi \mathrm{A}}=e^{\xi A} e^{\nu(\xi) V} e^{M_{\xi} \mathrm{aP}},
\end{aligned}
$$

where $M_{\mathfrak{u}}$ and $M_{v}$ are linear maps, so for example $M_{\mathfrak{u}} a \mathrm{P}=$ $\left[M_{u}\right]_{r}^{q} a^{r} P_{q}$ where $q, r$ are indices living in the radical. These identities follow from the Baker-Campbell-Hausdorff formula by applying the ideal property of the radical, $[\mathrm{T}, \mathrm{P}] \sim \mathrm{T}$ and closure of the $T$ 's and of the V's.

We don't really need the form of the $M_{\mathfrak{u}}$ matrix, but it can be computed explicitly from group theory. For example,

$$
u^{a}\left[T_{a}, P_{r}\right]=u^{a} f_{a r}^{q} P_{q} \Longrightarrow\left[M_{u}\right]_{r}^{q}=\exp \left(u^{a} f_{a r}^{q}\right),
$$

where $a$ is an index living in the simple algebra.

RADICAL FIRST. As usual, the transformation of $\ell_{R}$ is given by acting with some $g=e^{u \top} e^{a P}$ on $\ell_{R} S$ : 


$$
\begin{aligned}
& \tilde{\ell}_{R} S=e^{u \top} \underline{e^{a P} e^{z P}} e^{\xi A} S
\end{aligned}
$$

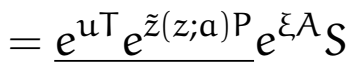

$$
\begin{aligned}
& =e^{\mathrm{M}_{\mathrm{u}} \tilde{z}(z ; \mathrm{a}) \mathrm{P}} \frac{e^{u \top}}{e^{\xi A}} S \\
& =e^{\mathrm{M}_{\mathrm{u}} \tilde{z}(z ; \mathrm{a}) \mathrm{P}} e^{\tilde{\xi}(\xi ; \mathfrak{u}) A} S \text {, }
\end{aligned}
$$

where we used closure of $\langle\mathrm{P}\rangle$, then braiding, then closure of $\langle T\rangle$, respectively. We denote by an underline where we inserted each property. To sum up, we have the following hierarchy for the transformation rules:

$$
z \rightarrow M_{\mathfrak{u}} \tilde{z}(z ; a), \quad \xi \rightarrow \tilde{\xi}(\xi ; u) .
$$

As we can see, the $z^{\prime}$ s transform completely by themselves and and they fully realize the group, that is, their transformation depends on the two group parameters a and $u$. The $\xi$ 's are therefore inessential.

SIMPLE FIRST. Same argument, but for clarity of notation we flip the order of the group element, $g=e^{\mathrm{aP}} e^{\mathrm{uT}}$, and have it act on $\ell_{\mathrm{L}} \mathrm{S}$ :

$$
\begin{aligned}
& \tilde{\ell}_{\mathrm{L}} \mathrm{S}=e^{\mathrm{aP}} e^{u \mathrm{u} T} e^{\xi A} e^{z \mathrm{P}} S \\
& =e^{a P} e^{\tilde{\xi}(\xi ; u) A} \underline{e^{\tilde{u}(u ; \xi) V} e^{z P} S}
\end{aligned}
$$

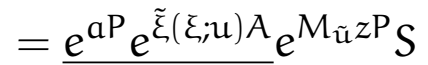

$$
\begin{aligned}
& =e^{\tilde{\xi}(\xi ; \mathfrak{u}) \mathrm{A}} e^{v(\xi ; \mathfrak{u}) \mathrm{V}} e^{\mathrm{M}_{\tilde{\xi}} \mathrm{aP}} e^{\mathrm{M}_{\tilde{u}} z \mathrm{P}} S \\
& =e^{\tilde{\xi}(\xi ; u) A} e^{\tilde{z}(z, \xi ; u, a) P} S \text {, }
\end{aligned}
$$

where we used closure of $\langle T\rangle$, then braiding, then braiding again, then closure of $\langle\mathrm{P}, \mathrm{V}\rangle$. The group action hierarchy is:

$$
\xi \rightarrow \tilde{\xi}(\xi ; u), \quad z \rightarrow \tilde{z}(z, \xi ; u, a) .
$$


We see the ' 's transform by themselves. However, they don't fully realize the group, as their transformation depends on $a$. This means some of the $z^{\prime}$ s (but not necessarily all) are essential; the details depends on the specific group. In the following section, we investigate a simple toy example involving equivalences with these two orderings.

\subsubsection{Example: Curve geometry}

In this example, Rachel and Leo are interested in drawing plane curves. For this, they are given the coset space $\operatorname{ISO}(2) /\{1\}$, where by $\{1\}$ we mean the trivial group with just the identity. They agree to employ diffeomorphism invariance and to eliminate any inessential degree of freedom. For simplicity, they will limit their actions to at most second order in derivatives, but as we will see later, this restriction can be relaxed. The conclusion will be that Rachel and Leo will find two different, inequivalent actions.

RACHEL'S THEORY . The group $\operatorname{ISO}(2)=\mathbb{R}^{2} \rtimes \mathrm{SO}(2)$ is semisimple, so Rachel employs the Levi ordering, in particular the radical one. She parametrizes the coset space by the lift $\ell_{R}=e^{x P_{1}} e^{y P_{2}} e^{\theta J}$; the three elements $\{x, y, \theta\}$ are functions of some diff parameter $\lambda$. Under action of an Euclidean element $\left(a^{i}, \varphi\right)$, she computes the transformation rules:

$$
\begin{aligned}
& x \rightarrow x \cos (\varphi)-y \sin (\varphi)+a^{1}, \\
& y \rightarrow y \cos (\varphi)+x \sin (\varphi)+a^{2}, \\
& \theta \rightarrow \theta+\varphi .
\end{aligned}
$$

Rachel sees that $\theta$ is inessential, because $x$ and $y$ transform among themselves and fully realize it. So she discards $\theta$. If she had used the Maurer-Cartan procedure, she would've found the inverse Higgs constraint $\tan (\theta) \partial_{\lambda} x=\partial_{\lambda} y$. In any case, she computes the action:

$$
S_{R}=\int \mathrm{d} \lambda \sqrt{\left(x^{\prime}\right)^{2}+\left(y^{\prime}\right)^{2}} \mathrm{P}\left(\frac{-y^{\prime} x^{\prime \prime}+x^{\prime} y^{\prime \prime}}{\left(\left(x^{\prime}\right)^{2}+\left(y^{\prime}\right)^{2}\right)^{3 / 2}}\right)
$$


for an arbitrary function P. Of course, we recognize this: it's the usual action for planar curves, with the extrinsic curvature,

$$
\kappa=\frac{-y^{\prime} x^{\prime \prime}+x^{\prime} y^{\prime \prime}}{\left(\left(x^{\prime}\right)^{2}+\left(y^{\prime}\right)^{2}\right)^{3 / 2}},
$$

being the diffeomorphic invariant intrinsic to the embedding. This action is really a procedure for drawing curves on paper. Rachel slides a ruler against the paper in a fixed direction. With the other hand, she holds a pen next to the ruler, allowing the pen to be pushed by it. Reparametrization invariance arises because the speed of the ruler can be removed as a degree of freedom; the only thing that changes the drawing is Rachel moving the pen up or down as its pushed across the paper left to right. For linear equations of motion obtained from $P(\kappa)=1$, she doesn't move the pen at all, only letting it be pushed by the ruler, and draws a straight line.

LEO'S THEORY . Leo also recognizes the nonsimplicity of $\operatorname{ISO}(2)$, but opts for the simple-first Levi ordering $\ell_{L}=e^{\theta \mathrm{J}} e^{\sigma \mathrm{P}_{2}} e^{\pi \mathrm{P}_{1}}$. His transformation laws are:

$$
\begin{aligned}
& \theta \rightarrow \theta+\varphi, \\
& \pi \rightarrow \pi+a^{1} \cos (\theta)+a^{2} \sin (\theta), \\
& \sigma \rightarrow \sigma+a^{2} \cos (\theta)-a^{1} \sin (\theta),
\end{aligned}
$$

He notices $\theta$ is essential, as it appears in all transformation rules. But it's not sufficient to fully realize the group, so he must add either $\pi$ or $\sigma$ to complete the problem. Either option leads to the same result, so he picks $\theta, \pi$ as his pair of essential bosons and throws away $\sigma$, which would be equivalent to the inverse Higgs constraint $\sigma \partial_{\lambda} \theta=\partial_{\lambda} \pi$. He derives the action:

$$
S_{\mathrm{L}}=\int \mathrm{d} \lambda \theta^{\prime} \mathrm{F}\left(\pi+\frac{-\pi^{\prime} \theta^{\prime \prime}+\theta^{\prime} \pi^{\prime \prime}}{\left(\theta^{\prime}\right)^{3}}\right),
$$




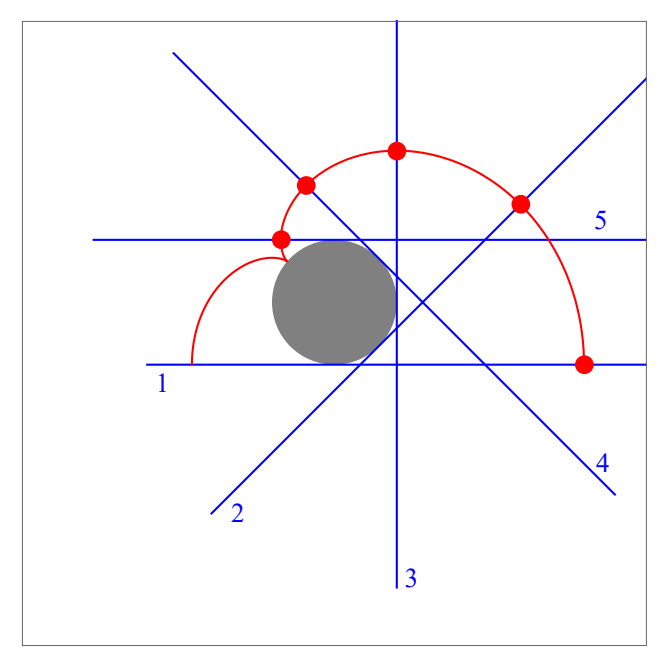

Figure 7.1: Leo's notion of curve drawing is to slide a ruler without slipping around a fixed circle, pushing the pen with the ruler. Here, Leo has kept the pen still with respect to the ruler. We draw a full rotation cycle, though we have included only five positions of the ruler to simplify the image.

for arbitrary F. This action is less known that the previous one; the diffeomorphic invariant is:

$$
\tau=\pi+\frac{-\pi^{\prime} \theta^{\prime \prime}+\theta^{\prime} \pi^{\prime \prime}}{\left(\theta^{\prime}\right)^{3}}
$$

This is also a prescription for drawing curves. Leo places a wheel together with a ruler on the paper. He then rotates the ruler without slipping around the fixed wheel. Again, the ruler's angular speed can be removed as a degree of freedom; only the pen's motion with respect to the ruler matters. For linear equations of motion obtained from $F(\tau)=\tau^{2}$, Leo simply lets the pen be pushed by the ruler and draws an involute of the wheel. See Figure 7.I for visualization. ${ }^{1}$

The invariant $\tau$ is a notion of torsion as it's measures the winding of the pen around the wheel and thus to the displacement of the pen from its original position after one rotation cycle. A curve that doesn't close after one cycle must necessarily have nonzero torsion. Note this torsion is different from the one of curves in ${ }_{3} \mathrm{D}$ space.

1 This is a drawing toy know as a spirograph. 
Rachel's and Leo's action are inequivalent and represent two distinct procedures for drawing curves on paper. Rachel and Leo started from the same coset space and employed the same prescription of removing inessential degrees of freedom, but arrived at inequivalent results.

Let's us verify this inequivalence. Notice that Rachel's y simply displays a constant shift under $\mathrm{P}_{1}$, that is, $y \rightarrow y+a^{1}$, but Leo's corresponding $\pi$ has a $\theta$-dependent transformation:

$$
\pi \rightarrow \pi+\mathrm{a}^{1} \cos \theta,
$$

and $\theta$ doesn't transform under $P_{1}$. Two try and match the two, we must redefine $\pi=\pi(\phi, \theta)$ so that $\phi$ simply shifts under $\mathrm{P}_{1}$ as well. We still have the diffeomorphism symmetry, so the definition of $\theta$ is ultimately irrelevant. To find the $\pi$ redefinition, we take the infinitesimal variation $\delta \pi(\phi, \theta)$ and impose the requirements we just presented, $\delta \phi=a^{1}$ and $\delta \theta=0$, leading to the equations,

$$
\partial_{\phi} \pi=\cos \theta,
$$

which are solved by $\pi=\phi \cos \theta+f(\theta)$ where $f$ is a free function. Now take Leo's torsion invariant and use the above solution for $\pi(\phi, \theta)$ to get:

$$
\begin{aligned}
\tau & =\pi+\frac{-\pi^{\prime} \theta^{\prime \prime}+\theta^{\prime} \pi^{\prime \prime}}{\left(\theta^{\prime}\right)^{3}}, \\
& =\frac{\cos \theta}{\left(\theta^{\prime}\right)^{2}} \phi^{\prime \prime}-\frac{2\left(\theta^{\prime}\right)^{2} \sin \theta+\theta^{\prime \prime} \cos \theta}{\left(\theta^{\prime}\right)^{3}} \phi^{\prime}+\left(f^{\prime \prime}(\theta)+f(\theta)\right)
\end{aligned}
$$

We still have the gauge freedom behind $\theta(\lambda)$ plus the free $f(\theta)$ which must be used so that Leo's torsion has the same mathematical form as Rachel's curvature:

$$
\kappa=\frac{y^{\prime \prime}}{\left[1+\left(y^{\prime}\right)^{2}\right]^{3 / 2}}
$$


where we picked the $x(\lambda)=\lambda$ gauge for Rachel to simplify the expression. At the same time, Rachel's and Leo's line elements also need to have the same mathematical form:

$$
\begin{aligned}
& \mathrm{d} s_{\mathrm{R}}=\sqrt{1+\left(\mathrm{y}^{\prime}\right)^{2}} \mathrm{~d} \lambda \\
& \mathrm{ds_{ \textrm {L } }}=\theta^{\prime} \mathrm{d} \lambda
\end{aligned}
$$

This is impossible; if we match the line elements then imposes $\theta=\int \mathrm{d} \lambda \sqrt{1+\left(y^{\prime}\right)^{2}}$ but this condition then leads to Leo's torsion becoming nonlocal, since $\theta$ appears explicitly without derivatives for him, and no choice of $f(\theta)$ can remove this nonlocality. This conclusion holds even if Rachel and Leo use higher derivatives in their theories, since it's based merely on the incompatibility of their line elements and fundamental invariants.

\subsection{HIERARCHIES IN FLAT SPACETIMES}

Here we discuss some issues with properly ordering the parametrization of a coset space in flat spacetime. That is, we study examples where some group is broken down to merely Poincaré, the isometry group of Minkowski space. Typically such breakdown will produce both scalar and vector Goldstones; in more exotic circumstances, even tensors can arise.

\subsubsection{Vectors: Conformal to Poincaré}

It's known that no vector Goldstone can exist in flat spacetime [65], that is to say, any potential vector Goldstone ends up being inessential. Let's us consider the hierarchy of one such example to understand what happens. A well studied symmetry breaking pattern in flat spacetime is that of the conformal group $\mathrm{SO}(2,4)$ down to Poincare $\mathrm{ISO}(1,3)$, meaning the breaking of dilations D and the special conformal transformation (SCT) $K_{\mu}$. Typically, the literature employs the coset ordering $[63,66]$,

$$
\ell=e^{x \cdot P} e^{\pi \mathrm{D}} e^{\xi \cdot k} .
$$


From this, one can derive that $\pi$ transforms without reference to $\xi^{\mu}$, but $\xi^{\mu}$ does with reference to $\pi$. Therefore, $\xi^{\mu}$ is inessential and can be removed; the resulting theory is given purely in terms of the dilaton $\pi$.

We will show this is not the preferred parametrization in the sense of Section 7.2.1. That is, there is a different one in which neither boson transforms with respect to the other. Unfortunately, the conformal group is simple and therefore we cannot employ the Levi decomposition to guide the coset ordering. At this juncture, we simply employ an educated guess. It turns out the ideal ordering is the following:

$$
\ell=e^{x \mathrm{P}} e^{\xi K} e^{\pi \mathrm{D}} .
$$

In this parametrization, $x^{\mu}$ and $\pi$ still transform as before. As for $\xi^{\mu}$, it transforms linearly under dilations (it didn't before); under the SCT, its transformation is a long expression. For simplicity we write down only the infinitesimal version:

$$
\delta_{b} \xi^{\mu}=b^{\mu}+2\left(\xi^{\mu} \chi^{\nu} b^{\rho}+b^{\mu} \chi^{\nu} \xi^{\rho}-x^{\mu} \xi^{v} b^{\rho}\right) \eta_{v \rho} .
$$

The important thing is that, overall, neither $\pi$ nor $\xi^{\mu}$ transform with respect to the other; this parametrization has no hierarchy. Let's construct the EFT using the Maurer-Cartan procedure. We determine its elements to be:

$$
\begin{aligned}
\omega_{\mathrm{P}}^{\alpha} & =e^{\pi} \delta_{\mu}^{\alpha} \mathrm{d} x^{\mu}, \\
\omega_{\mathrm{D}} & =\left(\partial_{\mu} \pi+2 \xi_{\mu}\right) \mathrm{d} x^{\mu}, \\
\omega_{\mathrm{K}}^{\alpha} & =e^{-\pi}\left(\partial_{\mu} \xi^{\alpha}+2 \xi^{\alpha} \xi_{\mu}-\xi^{2} \delta_{\mu}^{\alpha}\right) \mathrm{d} x^{\mu}, \\
\omega_{M}^{\alpha \beta} & =-4\left(\xi^{\alpha} \delta_{\mu}^{\beta}-\xi^{\beta} \delta_{\mu}^{\alpha}\right) \mathrm{d} x^{\mu} .
\end{aligned}
$$

For starters, we have the measure,

$$
\mathrm{dV}=\frac{1}{4 !} \epsilon^{\alpha \beta \gamma \delta} \omega_{\mathrm{P}}^{\alpha} \omega_{\mathrm{P}}^{\beta} \omega_{\mathrm{P}}^{\gamma} \omega_{\mathrm{P}}^{\delta}=e^{4 \pi} \mathrm{d}^{4} \chi
$$


Now, by writing $\mathrm{d} x^{\alpha}=e^{-\pi} \omega_{\mathrm{P}}^{\alpha}$ and inserting it into $\omega_{\mathrm{D}}$ and $\omega_{\mathrm{K}^{\prime}}^{\alpha}$ we get:

$$
\begin{aligned}
\omega_{\mathrm{D}} & =\nabla_{\alpha} \pi \omega_{\mathrm{P}}^{\alpha}, \\
{\left[\omega_{\mathrm{K}}\right]_{\beta} } & =\nabla_{\alpha} \xi_{\beta} \omega_{\mathrm{P},}^{\alpha} \\
\nabla_{\alpha} \pi & \stackrel{\text { def }}{=} e^{-\pi}\left(\partial_{\alpha} \pi+2 \xi_{\alpha}\right), \\
\nabla_{\alpha} \xi_{\beta} & \stackrel{\text { def }}{=} e^{-2 \pi}\left(\partial_{\alpha} \xi_{\beta}+2 \xi_{\alpha} \xi_{\beta}-\xi^{2} \eta_{\alpha \beta}\right) .
\end{aligned}
$$

The $\nabla_{\alpha} \pi$ and $\nabla_{\alpha} \xi_{\beta}$ are covariant under $\mathrm{SO}(1,3)$ —contract the $\alpha, \beta$ indices properly, and the result is $\mathrm{SO}(2,4)$-invariant. Furthermore, any constraint that is covariant or invariant might be applicable as an inverse Higgs constraint. In particular,

$$
\nabla_{\alpha} \pi=0 \Longrightarrow \xi_{\mu}=\frac{1}{2} \partial_{\mu} \pi
$$

So indeed, it seems we have the same conclusion as before: the $\xi^{\mu}$ is inessential and can be removed. Not so fast. We also know that $\xi^{\mu}$ transforms without reference to $\pi$, so it should also be possible to remove the dilaton. Indeed, this is so. Let us first anti-symmetrize $\nabla_{\alpha} \xi_{\beta}$; this is a Lorentz-covariant procedure, so it's allowed, and it protects us from ghost degrees of freedom in the vector:

$$
\nabla_{[\alpha} \xi_{\beta]}=e^{-2 \pi} \partial_{[\alpha} \xi_{\beta]} \stackrel{\text { def }}{=} e^{-2 \pi} F_{\alpha \beta} .
$$

The following normalization then acts as an inverse Higgs constraint:

$$
\begin{aligned}
& \nabla_{[\alpha} \xi_{\beta]} \nabla^{[\alpha} \xi^{\beta]}=\Lambda^{4} \\
& \Longrightarrow e^{4 \pi}=\frac{F^{2}}{\Lambda^{4}}
\end{aligned}
$$

for $\Lambda$ some arbitrary scale. This removes $\pi$, but now the only healthy term to use in the action is the measure $\wedge^{4} e^{4 \pi} \mathrm{d}^{4} x$, and the action takes the highly suspicious form:

$$
S=\int d^{4} F^{2}
$$


The coset construction seems to have glitched here, because it returned as output Maxwell's theory, whose vacuum doesn't break conformal symmetry! This is because of gauge invariance: the vacuum is completely and unambiguously specified merely by $F_{\mu \nu}=0$, without reference to $\xi_{\mu}$. The coset construction guaranteed that $\xi_{\mu}$ transformed non-linearly, but gauge invariance makes $\xi_{\mu}$ physically irrelevant. Another way to look at this, the action has an accidental U(1) symmetry that wasn't accounted for in the original coset parametrization. So the Maurer-Cartan form has no way of "knowing" what it's supposed to do with this symmetry.

In any case, we wouldn't really call $\xi_{\mu}$ a Goldstone, merely a standard matter field. The dilaton $\pi$ is still the only genuine Goldstone that arises from this symmetry breaking pattern. However, employing the ideal coset ordering helped us understand what really went wrong with the vector Goldstone. In the literature, it's often said that the vector is inessential because it's massive and this is independent of coset parametrization. The argument holds even in a different coset parametrization for which the IHC is diffferential, rather than algebraic, in $\xi^{\mu}$ (and thus not readily solvable): the fact $\xi^{\mu}$ is massive but $\pi$ isn't means the former gets integrated out at low energies. But this isn't quite true; our vector here is massless. On the other hand, the IHC $\xi_{\mu}=\partial_{\mu} \pi / 2$ still exists, yet the removal of $\xi^{\mu}$ can't be interpreted as it being integrated out.

The vector mass is an artifact of the parametrization; more fundamentally, the vector failed to be a Goldstone because its nonlinear symmetry was drowned by the gauge symmetry.

Here, we can't find a way to rescue the nonlinear vector symmetry. It's impossible to break $\mathrm{U}(1)$ while preserving $\mathrm{SO}(2,4)$ and still having a healthy theory, even when considering quasi-invariants. But, as we will later see in Section 7.4, the situation is different in curved spacetimes.

\subsubsection{Tensors: Special galileon}

We now discuss an example involving both inessential vectors and 2-tensors Goldstones, together with an essential scalar. Again, focusing on finding a preferred parametrization of the associated 
coset space will render the hierarchy between the bosons manifest. This will also be an opportunity to employ the various Levi decomposition techniques we presented in Section 2.2.

First, recall the flat spacetime galileon theory. This is a scalar field $\phi$ enjoying an extended shift symmetry $\phi \rightarrow \phi+c+b_{\mu} x^{\mu}$. We describe the Galileo group $\operatorname{Gal}(D, 1)$ in greater detail in Section A.1.2, but essentially it consists of enlarging the Poincare algebra by a scalar $\mathrm{Q}$ and vector $\mathrm{B}_{\mu}$, such that the only nontrivial additional commutator is:

$$
\left[\mathrm{P}_{\mu}, \mathrm{B}_{v}\right]=\eta_{\mu \nu} \mathrm{Q} .
$$

All other additional commutators are either zero or follow from tensorial structure. The scalar field realizes the breaking pattern $\mathrm{Gal}(\mathrm{D}, 1) \rightarrow \mathrm{ISO}(1, \mathrm{~d})$; together with the fact the origin of spacetime breaks translations yields the coset space $\mathrm{Gal}(\mathrm{D}, 1) / \mathrm{SO}(1, \mathrm{~d})$, with lift:

$$
\ell=e^{x \cdot P} e^{\xi \cdot B} e^{\phi Q}
$$

One then readily checks that $\phi$ transforms without reference to $\xi^{\mu}$, so that the latter is readily eliminated as an inessential field.

The theory we want to consider, however, is the special galileon [67]. It turns out the above algebra can be further expanded into that of the $S G a l(D, 1)$ group by adding a new symmetric tensor generator $S_{\mu \nu}$. The additional commutators, besides those that vanish or are given by tensorial structure, are $[67,68]$ :

$$
\begin{aligned}
{\left[\mathrm{P}_{\mu}, S_{\nu \rho}\right] } & =\eta_{\mu \nu} B_{\rho}+\eta_{\mu \rho} B_{v}-\frac{2}{D} \eta_{\mu \rho} B_{\mu} \\
{\left[B_{\mu}, S_{\nu \rho}\right] } & =\alpha^{2}\left(\eta_{\mu \nu} P_{\rho}+\eta_{\mu \rho} P_{v}-\frac{2}{D} \eta_{\mu \rho} P_{\mu}\right), \\
{\left[S_{\mu \nu}, S_{\rho \sigma}\right] } & =\alpha^{2}\left(\eta_{\mu \rho} M_{v \sigma}+\eta_{\mu \sigma} M_{\nu \rho}+\eta_{\nu \rho} M_{\mu \sigma}+\eta_{v \sigma} M_{\mu \rho}\right),
\end{aligned}
$$


where $\alpha$ is a free constant. In the literature, one then performs the construction by writing the $\operatorname{SGal}(\mathrm{D}, 1) / \mathrm{SO}(1, \mathrm{~d})$ lift:

$$
\ell=e^{x \cdot P} e^{\phi Q} e^{\xi \cdot B} e^{\frac{1}{2} \sigma \cdot S} .
$$

One can then check that under action of $S_{\mu v}$, the coordinates $x^{\mu}$ and vector $\xi^{\mu}$ mix with each other. Naively we would conclude this means the vector is now essential, since now the measure and any spacetime derivative will pick up factors involving $\xi^{\mu}$ when acting with $S_{\mu \nu}$. But that feels absurd, because when the group was just Gal $(1, D)$, we could write an action purely in terms of $\phi$. Then, we could've eliminated operators that break $\operatorname{SGal}(D, 1)$ in order to enhance the symmetry group of the action, without introducing the vector.

This intuition is correct. The action of the special galileon scalar in $(1+3) \mathrm{D}$ for example is given by:

$$
S=\int d^{4} x\left(-\frac{1}{2}(\partial \phi)^{2}+\frac{\alpha^{2}}{12}(\partial \phi)^{2}\left[(\square \phi)^{2}-\left(\partial_{\mu} \partial_{\nu} \phi\right)^{2}\right]\right),(7.62)
$$

with the additional nonlinear symmetry (here we give it in active form, as it's easier to check the above action is invariant this way):

$$
\delta \phi=s_{\mu \nu} \chi^{\mu} \chi^{\nu}+\alpha^{2} s^{\mu \nu} \partial_{\mu} \phi \partial_{\nu} \phi,
$$

where $s_{\mu \nu}$ is a traceless symmetric matrix of constant transformation parameters. So what went wrong? In terms of transformations, we declared $\xi^{\mu}$ to be essential, but clearly that's not true. The issue is the following. Finding a coset parametrization in which $x^{\mu}$ and $\phi$ transform by themselves is sufficient to show these are the essential objects of the action, but the converse is not true! Just because you found one in which $x^{\mu}, \phi$ and $\xi^{\mu}$ mix among themselves doesn't mean all three are essential. Just like in the previous section, we must alter the parametrization typically found in literature in order to find the preferred one in which the Goldstone hierarchy is made manifest. 
To begin with, what is the group SGal(D, 1$)$, really? Well, whenever we find a mysterious new group, our first instinct should be to Levi decompose it. The standard Galileo group decomposes into:

$$
\operatorname{Gal}(D, 1)=\exp \langle P, Q, B\rangle \rtimes S O(1, d),
$$

where \langle\rangle denotes span. The question then is, where does $S_{\mu v}$ fit into? Does it enlarge the radical or the simple factor? Or does it destroy the decomposition altogether, turning the group from nonsimple to simple?

First, we see that $\langle\mathrm{P}, \mathrm{Q}, \mathrm{B}\rangle$ continues to be an ideal, because $[\mathrm{P}, \mathrm{S}] \sim \mathrm{B},[\mathrm{Q}, \mathrm{S}]=0$ and $[\mathrm{B}, \mathrm{S}] \sim \mathrm{P}$, so that

$$
[\langle\mathrm{P}, \mathrm{Q}, \mathrm{B}\rangle, \mathrm{S}]=\langle\mathrm{P}, \mathrm{B}\rangle \subset\langle\mathrm{P}, \mathrm{Q}, \mathrm{B}\rangle,
$$

and of course it continues to be solvable. Thus it's still part of the radical; the addition of $S_{\mu \nu}$ doesn't convert the group into a simple one without a radical.

Meanwhile, $S_{\mu \nu}$ has a nontrivial commutation with itself: $[S, S] \sim$ $M$, for a Lorentz generator $M$. This means it can't be part of the radical as it would destroy the solvability condition. So it has to go into the simple factor. And since it commutes nontrivially with $M_{\mu v}$, it must genuinely enlarge $\mathrm{SO}(1, \mathrm{~d})$, instead of being just an additional semisimple factor. Classifying simple groups is easy, and we quickly notice this new enlarged simple factor is the special linear group SL(D). One way to intuit this is that the $S_{\mu \nu}$ transformations are parametrized by a traceless symmetric $s_{\mu \nu}$ matrix [67] and those of $M_{\mu \nu}$ by antisymmetric $\eta_{\mu \nu}$-the two together form any traceless matrix.

Ergo, the special Galileo group Levi decomposes as:

$$
\operatorname{SGal}(D, 1)=\exp \langle P, Q, B\rangle \rtimes \operatorname{SL}(D) .
$$

Now, there should be a choice of algebra basis (or, equivalently, a coset space parametrization) in which the radical transforms linearly under the simple factor, that is, the elements in $e^{x \cdot P} e^{\phi Q} e^{\xi \cdot B}$ 
transform in a representation. This already happens for $\phi$, of course, but for $x^{\mu}$ and $\xi^{\mu}$ it requires that $\langle\mathrm{P}\rangle$ and $\langle\mathrm{B}\rangle$ be each individually an ideal. This follows from the discussion in Section 7.2.1 involving the braiding identity for the product of exponentials:

$$
\begin{aligned}
& {[P, S] \sim P \Longrightarrow e^{s \cdot S} e^{x \cdot P}=e^{(\exp s) x \cdot P} e^{s \cdot S},} \\
& {[B, S] \sim B \Longrightarrow e^{s \cdot S} e^{\xi \cdot B}=e^{(\exp s) \xi \cdot B} e^{s \cdot S} .}
\end{aligned}
$$

The special Galileo algebra in (7.58) doesn't display the properties above; rather, we have $[\mathrm{P}, \mathrm{S}] \sim \mathrm{B}$ and $[\mathrm{B}, \mathrm{S}] \sim \mathrm{P}$. So the basis isn't the preferred one. Perform the following change:

$$
\begin{aligned}
\tilde{\mathrm{P}}_{\mu} & =\frac{1}{\sqrt{2}}\left(\alpha \mathrm{P}_{\mu}+\mathrm{B}_{\mu}\right), \\
\tilde{\mathrm{B}}_{\mu} & =\frac{1}{\sqrt{2}}\left(\mathrm{~B}_{\mu}-\alpha \mathrm{P}_{\mu}\right), \\
\tilde{\mathrm{Q}} & =\alpha \mathrm{Q}, \\
\tilde{S}_{\mu \nu} & =S_{\mu \nu} / \alpha .
\end{aligned}
$$

The algebra now is:

$$
\begin{aligned}
{\left[\tilde{\mathrm{P}}_{\mu,}, \tilde{\mathrm{B}}_{v}\right] } & =\eta_{\mu \nu} \tilde{\mathrm{Q}} \\
{\left[\tilde{\mathrm{P}}_{\mu}, \tilde{\mathrm{S}}_{v \rho}\right] } & =\eta_{\mu \nu} \tilde{\mathrm{P}}_{\rho}+\eta_{\mu \rho} \tilde{\mathrm{P}}_{v}-\frac{2}{\mathrm{D}} \eta_{\mu \rho} \tilde{\mathrm{P}}_{\mu}, \\
{\left[\tilde{\mathrm{B}}_{\mu}, \tilde{S}_{v \rho}\right] } & =-\eta_{\mu \nu} \tilde{\mathrm{B}}_{\rho}-\eta_{\mu \rho} \tilde{\mathrm{B}}_{v}+\frac{2}{\mathrm{D}} \eta_{\mu \rho} \tilde{\mathrm{B}}_{\mu,} \\
{\left[\tilde{\mathrm{S}}_{\mu \nu}, \tilde{\mathrm{S}}_{\rho \sigma}\right] } & =\eta_{\mu \rho} \mathrm{M}_{v \sigma}+\eta_{\mu \sigma} \mathrm{M}_{v \rho}+\eta_{\nu \rho} M_{\mu \sigma}+\eta_{v \sigma} M_{\mu \rho} .
\end{aligned}
$$

This now has the required ideal property for the braiding identity to be applied. That is, writing the coset lift as

$$
\ell=e^{x \cdot \tilde{\mathrm{P}}} e^{\phi \tilde{Q}} e^{\xi \cdot \tilde{\mathrm{B}}} e^{\frac{1}{2} \sigma \cdot S}
$$

guarantees that $x^{\mu}, \phi$ and $\xi^{\mu}$ each transform linearly as a multiplet by itself under $S_{\mu v}$, without mixing. The transformations 
under the radical $\langle P, Q, B\rangle$ and the Lorentz group $\mathrm{SO}(1, \mathrm{~d})$ remain unchanged. In terms of a group parameter

$$
g=e^{a \cdot \tilde{P}} e^{\frac{1}{2} \eta M} e^{c \tilde{Q}} e^{b \cdot \tilde{B}} e^{\frac{1}{2} s \cdot \tilde{s}},
$$

the dependencies of the transformation rules are found to be:

$$
\begin{aligned}
x^{\mu} & \rightarrow \tilde{x}^{\mu}(x ; a, \eta, s), \\
\phi & \rightarrow \tilde{\phi}(x, \phi ; c, b), \\
\xi^{\mu} & \rightarrow \tilde{\xi}^{\mu}(\xi ; \eta, b, s), \\
\sigma^{\mu \nu} & \rightarrow \tilde{\sigma}^{\mu \nu}(\sigma ; \eta, s) .
\end{aligned}
$$

Therefore we see that the minimal set of objects required to fully realize the group-meaning they have closed transformation rules and depend on all group parameters $\mathrm{a}^{\mu}, \mathrm{b}^{\mu}, \mathrm{c}, \eta^{\mu \nu}$ and $\mathrm{s}^{\mu \nu}$-is the pair $x^{\mu}$ and $\phi$. Thus, the $\xi^{\mu}$ and $\sigma^{\mu \nu}$ are inessential. This is precisely the preferred parametrization that renders the Goldstone hierarchy manifest.

We learn from this example that if the group admits a Levi decomposition, we must first parametrize the coset lift by one of the Levi-preferred orderings before we can conclude anything about the hierarchy between Goldstone bosons.

\subsection{HIERARCHIES IN CURVED SPACETIMES}

So far, we have only analyzed the Goldstone hierarchy of theories living in flat spacetime. It's time now to consider a curved spacetime. The situation of course is far more complicated, but the following example illustrates many of the features of such theories.

\subsubsection{Vectors: Poincaré to de Sitter}

We wish to consider a theory living in de Sitter spacetime, which means the unbroken subgroup ought to be $\mathrm{SO}(1, \mathrm{D})$. A straightforward example of a larger group containing this subgroup is the 
Poincaré group ISO(1, D). Hence, we will focus on the symmetry breaking pattern:

$$
\mathrm{ISO}(1, \mathrm{D}) \rightarrow \mathrm{SO}(1, \mathrm{D}) .
$$

This is not sufficient information for the coset construction. Recall we must specify not simply the unbroken subgroup that leaves the vacuum invariant, but also the stability subgroup that leaves the origin of spacetime invariant. But the origin of which spacetime?

1. The Poincaré group ISO $(1, \mathrm{D})$ is the isometry group of Minkowski spacetime $\mathbb{M}^{\mathrm{D}+1}$. Thus, we choose the origin of $\mathbb{M}^{\mathrm{D}+1}$. Its stability subgroup is $\mathrm{SO}(1, \mathrm{D})$.

2. The de Sitter group $\mathrm{SO}(1, \mathrm{D})$ is the isometry group of de Sitter spacetime $d S^{\mathrm{D}}$. Thus, we choose the origin of $d S^{\mathrm{D}}$. Its stability subgroup is $\mathrm{SO}(1, \mathrm{~d}) .^{2}$

The first choice means the coset construction for $\operatorname{ISO}(1, \mathrm{D}) / \mathrm{SO}(1, \mathrm{D})$ while the second for $\operatorname{ISO}(1, \mathrm{D}) / \mathrm{SO}(1, \mathrm{~d})$. The choices therefore aren't a priori equivalent.

This equivalence isn't actually a new thing. It could happen in flat spacetime as well, e. g., when breaking the conformal group to Poincaré: $\mathrm{SO}(2, \mathrm{D}) \rightarrow \operatorname{ISO}(1, \mathrm{~d})$. One could either pick the origin of Minkowski space or of anti-de Sitter space, by recalling that $\mathrm{SO}(2, \mathrm{D})$ is the isometry group of AdS. This represents the socalled choice of basis for this symmetry breaking pattern: the first is the conformal basis while the second is the AdS basis [63]. The actions, as output of the coset construction, are inequivalent in the sense there's no invertible field redefinition from one to the other. Nontrivially, however, the physics of the two are equivalent at the S-matrix level [69], which isn't surprising considering the AdS/CFT duality.

Back to de Sitter space. The first theory $\operatorname{ISO}(1, \mathrm{D}) / \mathrm{SO}(1, \mathrm{D})$ corresponds to the usual embedding of the $\mathrm{dS}$ hyperboloid in a higher

2 See A.1.5 for the full description of the de Sitter group; space translations and spacetime dilations move the origin. 
dimensional Minkowski spacetime, which is familiar in the literature [33]. The only degree of freedom are the scalar (transverse) fluctuations of the brane. Concretely, the action is of DBI type at lowest order in derivatives:

$$
S=\int d^{D} x \sqrt{-g} \sqrt{\pi^{2}+(\partial \pi)^{2}},
$$

where the metric $g_{\mu \nu}$ is a $d S^{D}$ metric.

The second theory $\operatorname{ISO}(1, \mathrm{D}) / \mathrm{SO}(1, \mathrm{~d})$ we will now build. The full group ISO $(1, D)$ isn't simple, so preferred orderings for the coset lift are given by the Levi decomposition. We denote by a superscript $\mathbb{M}$ the translation and Lorentz generators of $\mathrm{ISO}(1, \mathrm{D})$. The first Levi ordering is the one with the radical going first:

$$
\ell_{R}=e^{\chi^{\mu} P_{\mu}^{M}} e^{\pi P_{D}^{M}} e^{\eta^{\mu} M_{\mu D}^{M}} .
$$

This, however, also outputs a familiar theory. It consists of a Minkowski brane embedded in a Minkowski bulk [33]. Again, only the scalar fluctuations of the brane survive as degrees of freedom and the action is of DBI-type. At lowest derivative order, it is:

$$
S=\int d^{4} x \sqrt{1+(\partial \pi)^{2}}
$$

using the $\eta_{\mu \nu}$ metric of $\mathbb{M}^{\mathrm{D}}$.

There's another possibility. Let us denote by $P_{i}^{d S}, D^{d S}$ and $M_{\mu v}^{d S}$ the dS translations, dilations and boost/rotations, respectively; see Section A.1.5 to see how these can be written in terms of the Lorentz transformations $M_{\mu \nu}^{\mathbb{M}}$ and $M_{\mu D}^{\mathbb{M}}$. Then the simple-first Levi ordering is:

$$
\ell_{L}=e^{\chi^{i} P_{i}^{d S}} e^{t D^{d S}} e^{\xi_{\mu}^{\mu} P_{\mu}^{M}+\pi P_{D}^{M}} .
$$

This will give a different theory. Moreover, as we will see, it preserves both the vector and the scalar as degrees of freedom. Therefore, it follows by direct counting of degrees of freedom its 
S-matrix isn't equivalent to the previous two DBI theories we have considered so far, hence representing a genuine ambiguity of the coset construction.

To better look at the underlying hierarchy, we will complete the coset construction of this theory via the normalization procedure. We thus need the explicit transformation laws of all elements in the lift $\ell_{L}$, which follow by action of a group element $g$ through the group product:

$$
\ell_{\mathrm{L}} \cdot \mathrm{SO}(1, \mathrm{~d}) \rightarrow \tilde{\ell}_{\mathrm{L}} \cdot \mathrm{SO}(1, \mathrm{~d})=\mathrm{g} \cdot \ell \cdot \mathrm{SO}(1, \mathrm{~d}) .
$$

Representing the lift as a matrix, the computation is straightforward if a bit laborious; to simplify this step, we switch to conformal time $t=-\log (-\tau)$ where we set the Hubble constant to $1, H=1$. We encounter a slight hiccup in the result: $\xi_{\mu}$ doesn't transform at all under a dilation. This is annoying, since we would like all objects to transform covariantly under the unbroken subgroup $\mathrm{SO}(1, \mathrm{D})$. It's not unsurmountable; the normalization procedure doesn't demand covariance, though it significantly simplify the computation if there. But with an educated (if ad hoc) guess, we notice that redefining $\xi_{\mu} \stackrel{\text { def }}{=} \xi_{\mu} / \tau$ gives the correct covariant transformation law for a vector under a dilation.

So the result is that, under the simple part of the group, the unbroken $\mathrm{SO}(1, \mathrm{D})$, the coordinates $x^{\mu}=\left(\tau, x^{i}\right)$ transform as in the isometries of (flat slicing) de Sitter space-full expressions for these are provided in Appendix B-while $\pi$ behaves as a scalar and $A_{\mu}$ as a vector.

On the other hand, under the radical part of the group, the broken translations $\mathbb{R}^{\mathrm{D}+1}$, we have:

$$
\begin{aligned}
x^{\mu} & \rightarrow x^{\mu}, \\
\pi & \rightarrow \pi-\theta, \\
A_{\mu} & \rightarrow A_{\mu}+\partial_{\mu} \theta, \\
\theta & \stackrel{\text { def }}{=} \frac{1}{\tau}\left(c+b^{i} x^{j} \delta_{i j}+\frac{1}{2} a x^{\mu} \chi^{\nu} \eta_{\mu \nu}\right),
\end{aligned}
$$


with $a, b^{i}$ and $c$ the $D+1$ translation parameters. The attentive reader will immediately notice the similarity between transformations (7.90) and (7.91) and those of the $\mathrm{U}(1)$ gauge connection and Stückelberg field we saw in Section 5.3. Indeed our $\pi$ here looks suspiciously like the longitudinal (Stückelberg) mode of the vector $A_{\mu}$. But remember, we don't want to impose invariance under gauged $\mathrm{U}(1)$, that is to say, for any choice of $\theta$, but only under the $\theta$ described above. In fact, inspired by what we saw in Section 7.3.1, we have to be particularly careful to avoid endowing the action with an accidental $U(1)$ gauge symmetry, or the coset construction might glitch again and convert the $A_{\mu}$ from a Goldstone vector with a genuine nonlinear symmetry into a boring gauge vector.

As a remark, it proves convenient to notice the following identity:

$$
\left[\nabla_{(\mu} \nabla_{v)}+g_{\mu \nu}\right] \theta=0,
$$

for $\nabla_{\mu}$ and $g_{\mu \nu}$ the usual Levi-Civita covariant derivative and the dS metric in flat slicing, conformal time coordinates, with $\theta$ being treated as a scalar in this context.

Notice the lack of hierarchy in this problem! Either $\pi$ or $A_{\mu}$ transforms by itself and they both fully realize the group. In principle we could keep either; removing the vector while keeping the scalar would give some sort of Galileon in dS [37, 66]. For this example, we will try to keep both.

Following the recipe, putative invariants are obtained by taking $x^{\mu}, \pi$ and $A_{\mu}$ and transforming them under some group element $g_{*}$ that we will fix momentarily:

$$
\begin{gathered}
\chi^{\alpha} \stackrel{g_{*}}{\rightarrow} \chi^{\alpha}, \\
\pi \stackrel{g_{*}}{\rightarrow} \Pi, \\
A_{\beta} \stackrel{g_{*}}{\rightarrow} \mathcal{A}_{\beta} .
\end{gathered}
$$

As usual, we want to set these objects to the origin of spacetime (which, in conformal time, is $(-1,0)$ ) and of fieldspace (the vacuum). Thus, we choose to set $X^{0}=-1, X^{i}=0, \Pi=0$ and $\mathcal{A}_{\mu}=0$. These normalizations then fix the Minkowski translations $a, b^{i}$ and $c$ together with the de Sitter dilations $\Lambda$ and translations $d^{i}$ : 


$$
\begin{array}{r}
\mathrm{a}_{*}=\mathrm{A}_{0}+\pi, \quad\left[\mathrm{b}_{\mathrm{i}}\right]_{*}=\mathrm{A}_{\mathrm{i},} \quad \mathrm{c}_{*}=\frac{1}{2}\left(\mathrm{~A}_{0}-\pi\right), \\
\Lambda_{*}=-\frac{1}{\tau} \quad \mathrm{d}_{*}^{\mathrm{i}}=-\chi^{\mathrm{i}} .
\end{array}
$$

We still haven't fully fixed $g_{*}$ because the group parameters of the stability subgroup $\mathrm{SO}(1, \mathrm{~d})$ consisting of de Sitter boosts/rotations remain. However, all objects transform covariantly under this group, so we needn't fix those parameters as long as we treat the flat $\alpha, \beta$ indices covariantly.

The $X^{0}, X^{i}, \Pi$ and $\mathcal{A}_{\alpha}$ consist of the zeroth order invariants. We have exhausted them by using their normalizations to fix $g_{*}$, so now we move on to invariants first order in derivatives. We simply take $\partial_{\mu} \pi$ and $\partial_{\nu} A_{\mu}$ and transform them under $g_{*}$ :

$$
\begin{aligned}
\partial_{\alpha} \pi \stackrel{g_{*}}{\rightarrow} \frac{\mathrm{d} \Pi}{\mathrm{d} X^{\alpha}}, \\
\partial_{\alpha} A_{\beta} \stackrel{g_{*}}{\rightarrow} \frac{\mathrm{d} \mathcal{A}_{\beta}}{\mathrm{d} X^{\alpha}},
\end{aligned}
$$

Similarly, we need an invariant one-form for the purposes of a measure. We take $\mathrm{d} x \mu$ and transform it:

$$
\mathrm{d} x^{\alpha} \stackrel{g_{*}}{\rightarrow} \mathrm{d} X^{\alpha} .
$$

Taking $\mathrm{d} X^{\alpha}, \mathrm{d} \Pi / \mathrm{d} X^{\alpha}$ and $\mathrm{d} \mathcal{A}_{\beta} / \mathrm{d} X^{\alpha}$ and plugging in the $g_{*}$ we found previously yields:

$$
\begin{aligned}
\mathrm{d} X^{\alpha} & =\frac{1}{\tau} \delta_{\mu}^{\alpha} \mathrm{d} x^{\mu}, \\
\frac{\mathrm{d} \Pi}{\mathrm{d} X^{\alpha}} & =\tau \delta_{\alpha}^{\mu}\left(A_{\mu}+\partial_{\mu} \pi\right), \\
\frac{\mathrm{d} \mathcal{A}_{\beta}}{\mathrm{d} X^{\alpha}} & =\tau^{2} \delta_{\alpha}^{\mu} \delta_{\beta}^{\nu}\left(\nabla_{\mu} A_{\nu}-\pi g_{\mu \nu}\right) .
\end{aligned}
$$


These objects, with the $\alpha, \beta$ indices, live in flat spacetimes and thus we must contract them with the flat metric $\eta^{\alpha \beta}$ or the permutation symbol $\epsilon^{\alpha_{1} \alpha_{2} \ldots}$. Indeed, we see the invariant volume is:

$$
\mathrm{dV}=\frac{1}{\mathrm{D} !} \varepsilon_{\alpha_{1} \alpha_{2} \ldots} \mathrm{d} X^{\alpha_{1}} \wedge \mathrm{d} X^{\alpha_{2}} \wedge \ldots=\frac{\mathrm{d}^{\mathrm{D} x}}{\tau^{\mathrm{D}}}
$$

For ease of notation, it's convenient to recast the remaining objects in terms of objects living in the curved de Sitter spacetime. For this, we recall that the prefactor of $\mathrm{d}^{\alpha}$ acts as a tetrad, the "square root" of the metric:

$$
\left(\frac{1}{\tau} \delta_{\mu}^{\alpha}\right)\left(\frac{1}{\tau} \delta_{v}^{\beta}\right) \eta_{\alpha \beta}=g_{\mu v}
$$

where $g_{\mu \nu}$ is the de Sitter metric in flat slicing, conformal time coordinates, $g_{\mu \nu}=\eta_{\mu \nu} / \tau^{2}$. This lets us rewrite the things in a more familiar manner:

$$
\begin{aligned}
\mathrm{d} V & =\mathrm{d}^{\mathrm{D}} x \sqrt{-\mathrm{g}}, \\
\mathrm{V}_{\mu} & =A_{\mu}+\partial_{\mu} \pi, \\
\mathrm{F}_{\mu v} & =\partial_{[\mu} A_{v]}, \\
S_{\mu v} & =\nabla_{(\mu} A_{v)}-\pi g_{\mu v},
\end{aligned}
$$

where we have split (7.104) into its antisymmetric part $F_{\mu \nu}$ and its symmetric part, while (7.103) we have renamed as $V_{\mu}$. Now the indices $\mu, v$ are world indices; they must be contracted with $g^{\mu v}$ or $\epsilon^{v_{1} v_{2} \ldots}$. We also notice that $V_{\mu}$ and $F_{\mu \nu}$ display a $U(1)$ gauge invariance, which wasn't part of the constructions, but $S_{\mu \nu}$ does not.

Notice that, by symmetrizing $\nabla_{\mu} V_{v}$, we can rewrite $S_{\mu \nu}$ without any explicit reference to $A_{\mu}$ :

$$
S_{\mu \nu}=\nabla_{(\mu} V_{v)}-\left[\nabla_{(\mu} \nabla_{v)}+g_{\mu \nu}\right] \pi
$$

Since $S_{\mu \nu}$ and $\nabla_{(\mu} V_{v)}$ are covariant, the last term in the equation above must be covariant as well:

$$
\mathrm{H}_{\mu \nu} \stackrel{\text { def }}{=}\left[\nabla_{(\mu} \nabla_{v)}+g_{\mu v}\right] \pi \text {. }
$$


Thus, to fully capture all operators in the EFT, we can use either the triplet $\left(V_{\mu}, S_{\mu \nu}\right)$ or $\left(V_{\mu}, H_{\mu \nu}\right)$, together with $\nabla_{\mu}$. Notice $F_{\mu \nu}$ is implicitly included given $V_{\mu}$ and $\nabla_{\mu}$.

We choose the latter option, $\left(V_{\mu}, H_{\mu \nu}\right)$. But $H_{\mu \nu}$ contains second derivatives of the scalar $\pi$ and therefore must appear in special combinations that prevent the propagation of ghosts [37, 70]. For simplicity, we will restrict the action to only leading order in derivatives, thus discarding $\mathrm{H}_{\mu \nu}$.

Similarly, $V_{\mu}$ can only appear in special combinations that prevent the propagation of ghosts, that is to say, a vector should have only d degrees of freedom, not $\mathrm{D}$. These combinations correspond to the so-called generalized Proca theories, derived for curved spacetimes in $[71,72]$. Here, we merely need to specialize to the de Sitter case.

So due to these considerations, the Lagrangian is given by:

$$
\mathcal{L}_{\text {gen.Proca }}\left(V_{\mu} ; \nabla_{\mu}\right)
$$

the mathematical structure of which is described in [71, 72]. Since $V_{\mu}=A_{\mu}+\partial_{\mu} \pi$, we decompose the action in the usual Stückelberg procedure. We simply have to define the generalized Stückelberg Lagrangian, $\mathcal{L}_{\text {gen.Stück }}\left(A_{\mu}, \partial_{\mu} \pi\right) \stackrel{\text { def }}{=} \mathcal{L}_{\text {gen.Proca }}\left(A_{\mu}+\partial_{\mu} \pi\right)$, so that the strictly invariant action then is:

$$
S=\int d^{D} x \sqrt{-g} \mathcal{L}_{\text {gen.Stück }}\left(A_{\mu}, \partial_{\mu} \pi ; \nabla_{\mu}\right) .
$$

Unfortunately, we are back to the same issue as in Section 7.3.1: this action has an accident $U(1)$ gauge symmetry that was never accounted for in the original coset construction. While it's certainly ISO (1, D) invariant, this global group has been drowned by the infinite $U(1)$ group. The vector $A_{\mu}$ doesn't physically realize any nonlinear symmetry; it's merely a gauge vector. But differently from the outcome in flat spacetime, here we can rescue the vector, by using quasi-invariants that break $\mathrm{U}(1)$ but not $\operatorname{ISO}(1, \mathrm{D})$. 


\subsubsection{Adding quasi-invariants}

As discussed in 4.5, quasi-invariants are the operators that, while not symmetric, shift by merely a total derivative and are thus still permitted in the action. Following the tools described there, we can derive the ones for this theory. There are $\mathrm{D}$ such objects; for the sake of expediency we will simply write down the first three, as their derivations and expressions quickly become laborious. Restoring the Hubble constant $\mathrm{H}$, they are:

$$
\begin{aligned}
& W_{1}=\pi, \\
& W_{2}=(\partial \pi)^{2}-D^{2} H^{2}, \\
& W_{3}=(\square \pi)\left[(\partial \pi)^{2}-(D-1) H^{2} \pi^{2}\right]-\frac{2}{3} D(D-1) H^{4} \pi^{3} .
\end{aligned}
$$

Of these, $W_{1}$ is a tadpole we would rather avoid in the action, so we can interpret $\pi$ merely as a background fluctuation rather than sourcing the background itself. The $W_{3}$ and higher Wess-Zumino terms are second order in derivatives, which we are avoiding in this example. Notice they look very much like the usual galileon Wess-Zumino terms of flat spacetime, and indeed become them in the group contraction $\mathrm{H} \rightarrow 0[37,73]$

We are thus left to work with merely $W_{2}$. It seems like a sick term, for its kinetic term has the wrong sign compared to the mass term. But this need not be a problem; after all, $(\partial \pi)^{2}$ also appears in the generalized Stückelberg Lagrangian we built before. We merely have to combine the two into something with the proper sign. So, we extract the mass term $-\left(\mathrm{m}^{2} / 2\right)\left(A_{\mu}+\partial_{\mu} \pi\right)^{2}$ from $\mathcal{L}_{\text {gen.Stück }}$ and add to it $\mathrm{qm}^{2} \mathrm{~W}_{2}$, for $\mathrm{q}$ some arbitrary dimensionless constant. It also proves convenient to perform the canonical normalization:

$$
\pi_{\mathrm{c}} \stackrel{\text { def }}{=} \mathrm{m} \sqrt{1-\mathrm{q}} \pi .
$$

With this these considerations, we take the action,

$$
S=\int d^{D} x \sqrt{-g}\left[\mathcal{L}_{\text {gen.Stück }}+q m^{2} W_{2}\right],
$$


and expand it:

$$
\begin{array}{r}
S=\int \mathrm{d}^{\mathrm{D}} x \sqrt{-\mathrm{g}}\left[-\frac{1}{4} \mathrm{~F}^{2}-\frac{1}{2} \mathrm{~m}^{2} A^{2}-\frac{\mathrm{m}}{\sqrt{1-\mathrm{q}}}(A \cdot \partial) \pi_{\mathrm{c}}+\mathcal{L}_{\text {gen.Stück }}^{\text {int }}\right. \\
-\frac{1}{2}\left(\partial \pi_{\mathrm{c}}\right)^{2}-\frac{1}{2}\left(\frac{\mathrm{q}}{1-\mathrm{q}}\right) \mathrm{DH}^{2} \pi_{\mathrm{c}}^{2}, \quad(7 \cdot 120)
\end{array}
$$

where $\mathcal{L}_{\text {gen.Stück }}^{\text {int }}$ collects all interaction terms of the generalized Stückelberg Lagrangian. For the mass term to be healthy, we require $0 \leqslant \mathrm{q}<1$. At the same time, if $\mathrm{q}=0$, then the crucial quasiinvariant that is breaking $\mathrm{U}(1)$ vanishes. Therefore, $0<\mathrm{q}<1$.

What he have achieved here is that, merely by changing the coset space parametrization from one Levi-preferred ordering to the other, we have changed the physics. One theory was a Minkowski brane in Minkowski bulk, while the other consists of a vector and scalar in de Sitter space enjoying a form of extended shift symmetry. Furthermore, the vector is indeed a Goldstone with a nonlinear symmetry, as the theory displays no gauge invariance-this latter point is important as it's unique to curved spacetimes and can't be achieved in Minkowski. And, of course, the number of degrees of freedom in the two theories don't match, so their S-matrices are automatically inequivalent.

\section{7·4.1.2 No inessential Goldstones}

This problem lacks any hierarchy between Goldstones: either $\pi$ or $A_{\mu}$ can be eliminated in favor of derivatives of the other. In terms of the normalization procedure, we could've return to the transformations (7.90) and (7.91) and recalled that the $\pi$ doesn't mix with the $A_{\mu}$ and vice-versa, and either fully realize the group. We could've eliminated either and straightforwardly derived invariants using only one of them. We can also look at this phenomenon from the inverse Higgs constraint (IHC) point of view. The IHCs here are not equivalent to integrating out the field through its equation of motion.

To remove $A_{\mu}$, we choose the following covariant normalization:

$$
V_{\mu}=0 \Longrightarrow A_{\mu}=-\partial_{\mu} \pi
$$


Alternatively, we can eliminate $\pi$ through invariant normalization of $g^{\mu \nu} S_{\mu v}$ :

$$
S=0 \Longrightarrow \pi=\frac{1}{D} \nabla_{\mu} A^{\mu} .
$$

Pure scalar theories with shift symmetries in de Sitter are discussed in [70], while [74] discusses similar ideas with vectors. Our conclusion here is that, differently from the situation in flat spacetime, an EFT with both scalar and vector Goldstones, with neither being removed in favor of the other, is possible. Inverse Higgs constraints exist as a mathematical possibility, but aren't demanded by physics. The problem thus has no Goldstone hierarchy. 


\section{$7 \cdot 5$ Discussion}

While the breaking of internal symmetries is well understood, that of spacetime ones leads to more peculiar phenomena. Chief among them are the so-called inessential Goldstone bosons which are associated with broken generators yet fail to appear in the low energy action altogether. In this chapter, we presented various techniques to study this hierarchy between essential and inessential bosons and constructed various examples to highlight key features.

Goldstone bosons live in coset spaces, so how one parametrizes this space, that is to say, chooses to order the group elements, is significant. We discussed how a nontrivial Levi decomposition for a group leads to two preferred parametrization, based on the orderings of the radical and semisimple elements, each of the two lead to a different hierarchical structure for the bosons. It would be interesting to see if other preferred orderings besides the Levi ones exist, or prove that they don't.

We used these techniques to re-examine two examples found in the literature. The first concerns breaking conformal symmetry down to Poincaré. In this case, we showed that with a different parametrization both bosons transform independently of each other, so a priori there is no hierarchy, but the massless vector fails to be a Goldstone due to its gauge symmetry. We also considered an example with tensor Goldstones, breaking the special Galileo to Poincaré, to highlight how to make hierarchies manifest through the Levi decomposition of a group extension.

Finally, we constructed an example living in de Sitter spacetime where both a scalar and a vector Goldstones were permitted to co-exist, this time because the gauge symmetry can be removed. This is a unique feature of curved spacetime and has no analogue in the $\mathrm{H} \rightarrow 0$ limit. This theory is directly inequivalent to others displaying the same symmetry breaking pattern by simple counting of the number of degrees of freedom, highlight the richness of structure for the EFT of broken curved spacetime symmetries. It would be interesting in the future to look at UV completions of such scalar-vector theories in de Sitter, which appear to be low energy limit of massive gravity theories. 

Part IV

CONCLUSION 



\section{CONCLUSION}

The spontaneous breaking of spacetime symmetries is a fecund ingredient for the construction of effective field theories for gravitational systems. In this thesis, we discussed the tools, both groupand field-theoretical, used in analyzing and constructing these EFTs, employing them to better understand the structure of Goldstones bosons for these symmetry breaking patterns and to better understand a specific example of cosmic inflationary theories.

In Part ii, we introduced the tools that we used in this thesis. In addition to the usual Maurer-Cartan formalism, we adapted a protocol from the mathematics literature and presented the normalization procedure as a novel way of constructing EFTs from their symmetry breaking pattern. We also extended this for theories with diffeomorphism symmetries, an essential component for gravity.

In Part iii, we employed these tools for more specific cases. Broken shift symmetries are often invoked to explain aspects of the embedding of inflation in string theory, yet inflation itself is the theory of broken time diffeomorphisms. The interplay between these two forms the basis of our study in shift-symmetric cosmologies. We analyzed the precise consequences of these broken theories in the associated EFT, from the more fundamental aspects down to phenomenological consequences.

Continuing in the same Part, another case we studied was the notion of inessential Goldstone bosons for broken spacetime symmetries. These are bosons that are not present in the low energy spectrum of the EFT. We scrutinized this phenomenon in flat spacetime, where scalars are the only essential bosons known to exist, then moved to curved spacetime, where we showed vectors can also be essential, with no equivalent in the flat limit. Along the way, we examined issues with uniqueness of the construction of EFTs.

Spontaneous symmetry breaking is a flexible and robust subject in physics, and effective field theory even more so. In this thesis, we expanded the knowledge of the field with new techniques and 
considerations for the discipline, as a complete understanding of broken spacetime symmetries is still an ongoing subject of research. Besides further work in the aspects presented in this thesis's case studies, these tools could be applied to completely unrelated gravitational systems that we haven't discussed here, such as dark matter and dark energy, as is natural for general methodologies in physics. 
Part V

APPENDIX 



\section{A.I FINITE GROUPS}

By Ado's theorem, the identity-connected component of any finite Lie group is some subgroup of the general linear group. As such, any Lie algebra admits a matrix representation. Utilizing this dramatically simplifies most group-theoretical computations, as computer algebra software can readily work with matrices.

Simple and compact Lie groups are completely classified and their matrix representations are well known. Spacetime groups also admit such matrices for their generators. Here we provide the commutation relations defining various algebras used in this work as well as the matrices that realize these commutators. If no commutation relation is provided between two generators, the trivial one is assumed.

For each group, we also provide its Levi decomposition. When the radical isn't immediately recognizeable as a known group, we simply denote it in terms of the span \langle\rangle of its generators.

We remind the reader $D$ denotes the number of spacetime dimensions, while $d=D-1$.

\section{A.1.1 Poincaré group}

Perhaps the most important spacetime symmetry group, the Poincare group $\operatorname{ISO}(1, d)$ is the isometry group of flat space. Its generators consist of spacetime translations $P_{\mu}$ and boosts/rotations $M_{\mu \nu}$.

Algebra

$\left[P_{\mu}, M_{v \rho}\right]=\eta_{\mu[v} P_{\rho]} \quad\left[M_{\mu \nu}, M_{\rho \sigma}\right]=M_{\rho[\mu} M_{v] \sigma}-M_{\sigma[\mu} M_{v] \rho}$. 
Matrices

$$
a^{\mu} P_{\mu}+\frac{1}{2} \epsilon^{\mu \nu} M_{\mu \nu}=\left[\begin{array}{cc}
\epsilon^{\mu \nu}\left(M_{\mu \nu}\right)_{\beta}^{\alpha} & a^{\mu} \\
0 & 0
\end{array}\right],
$$

where $\epsilon^{\mu \nu}$ is an antisymmetric matrix and $\left(M_{\mu \nu}\right)_{\beta}^{\alpha}$ the usual matrix representation of $\mathrm{SO}(1, \mathrm{~d})$.

Levi decomposition

$$
\operatorname{ISO}(1, d)=\mathbb{R}^{\mathrm{D}} \rtimes \mathrm{SO}(1, \mathrm{~d}) .
$$

\section{A.1.2 Galileo group}

The Galiliean group Gal(1, D) is a contraction of $\mathrm{ISO}(1, \mathrm{D})$. Considering Minkowski spacetime in $(\mathrm{D}+1)$ dimensions, we rescale the first $\mathrm{D}$ coordinates $x^{\mu} \rightarrow \mathrm{c}_{\mathrm{D}} x^{\mu}$ and rewrite all the dimensionless rapidites and angles for the Lorentz transformations involving the Dth dimension as $\varphi=v / c_{D}$. In the limit of infinite "speed" $c_{D} \rightarrow \infty$, the Poincare group contracts to Galileo [75].

It inherits the Poincaré generators $P_{\mu}$ and $M_{\mu \nu}$ for the $\chi^{\mu}$ coordinates, while $P_{D}$ and $M_{\mu D}$ become $Q$ and $B_{\mu}$, respectively after the contraction.

Algebra

$$
\begin{gathered}
P_{\mu} \text { and } M_{\mu v} \text { are those of } \operatorname{ISO}(1, d) \\
B_{\mu} \text { is covariant under } M_{\mu v}, \\
{\left[P_{\mu}, B_{v}\right]=\eta_{\mu \nu} Q}
\end{gathered}
$$

Matrices

$$
a^{\mu} P_{\mu}+\frac{1}{2} \epsilon^{\mu v} M_{\mu \nu}+c Q+b^{\mu} B_{\mu}=\left[\begin{array}{ccc}
\epsilon^{\mu \nu}\left(M_{\mu \nu}\right)_{\beta}^{\alpha} & 0 & a^{\mu} \\
b_{\mu} & 0 & c \\
0 & 0 & 0
\end{array}\right],
$$


where $\epsilon^{\mu \nu}$ is an antisymmetric matrix and $\left(M_{\mu \nu}\right)_{\beta}^{\alpha}$ the usual matrix representation of $\mathrm{SO}(1, \mathrm{~d})$.

Levi decomposition

$$
\operatorname{Gal}(1, D)=\exp (\langle\mathrm{P}, \mathrm{Q}, \mathrm{B}\rangle) \rtimes \mathrm{SO}(1, \mathrm{~d}) .
$$

\section{A.1.3 Carroll group}

The Carroll group Car(1, D) is also contraction of $\operatorname{ISO}(1, \mathrm{D})$. The procedure is identical to the Galilean contraction, but taking the limit $c_{D} \rightarrow 0$ instead [76].

Once again, the Poincaré generators $P_{\mu}$ and $M_{\mu \nu}$ for the $x^{\mu}$ coordinates remain unchanged, while $P_{D}$ and $M_{\mu D}$ become $Q$ and $\mathrm{B}_{\mu}$ after contracting.

Algebra

$$
\begin{gathered}
P_{\mu} \text { and } M_{\mu \nu} \text { are those of } \operatorname{ISO}(1, d) \\
V_{\mu} \text { is covariant under } M_{\mu \nu}, \\
{\left[Q, V_{v}\right]=\eta_{\mu \nu} Q .}
\end{gathered}
$$

Matrices

$$
a^{\mu} P_{\mu}+\frac{1}{2} \epsilon^{\mu \nu} M_{\mu \nu}+c Q+b^{\mu} V_{\mu}=\left[\begin{array}{ccc}
\epsilon^{\mu \nu}\left(M_{\mu \nu}\right)_{\beta}^{\alpha} & b^{\mu} & a^{\mu} \\
0 & 0 & c \\
0 & 0 & 0
\end{array}\right],
$$

where $\epsilon^{\mu \nu}$ is an antisymmetric matrix and $\left(M_{\mu \nu}\right)_{\beta}^{\alpha}$ the usual matrix representation of $\mathrm{SO}(1, \mathrm{~d})$.

Levi decomposition

$$
\operatorname{Car}(1, D)=\exp (\langle\mathrm{P}, \mathrm{Q}, \mathrm{V}\rangle) \rtimes \mathrm{SO}(1, \mathrm{~d}) .
$$




\section{A.1.4 Conformal group}

The Minkowski conformal group, $\mathrm{SO}(2, \mathrm{~d})$, is the group of all transformations that leave angles in Minkowski space invariant. ${ }^{1}$ It is also the isometry group of anti-de Sitter space, the spacetime of negative cosmological constant.

The conformal generators are the Poincare generators $P_{\mu}$ and $M_{\mu \nu}$ together with spacetime dilations $D$ and special conformal generator $K_{\mu}$.

Algebra

$$
\begin{aligned}
P_{\mu} \text { and } M_{\mu \nu} & \text { are those of } \operatorname{ISO}(1, d), \\
K_{\mu} & \text { is covariant under } M_{\mu v}, \\
{\left[P_{\mu}, D\right] } & =P_{\mu} \\
{\left[K_{\mu}, D\right] } & =-K_{\mu} \\
{\left[P_{\mu}, K_{v}\right] } & =2 M_{\mu \nu}-2 \eta_{\mu \nu} D
\end{aligned}
$$

\section{Matrices}

As the conformal group is $\mathrm{SO}(2, \mathrm{~d})$, one can use the usual matrix representations for Lorentz generators. The group $\mathrm{SO}(2, \mathrm{~d})$ acts on a $(D+2)$-dimensional spacetime; we index with $\mu$ the usual D coordinates, the additional time coordinate with - and the additional space coordinate with + .

$$
\begin{aligned}
P_{\mu} & =M_{-\mu}+M_{\mu+} \\
D & =M_{-+} \\
K_{\mu} & =M_{-\mu}-M_{\mu+} \\
M_{\mu \nu} & \text { unchanged }
\end{aligned}
$$

Levi decomposition

$\mathrm{SO}(2, \mathrm{D})$ is already simple.

1 More formally, it's the set of transformations that map the Minkowski metric $\eta_{\mu \nu} \rightarrow f(x) \eta_{\mu \nu}$, for $f(x)$ some function. 


\section{A.1.5 de Sitter group}

The de Sitter group SO $(1, D)$ is the isometry group of a spacetime with positive cosmological constant. It is thus of immense importance to both early and late cosmology, due to inflation and dark energy, respectively.

Its generators consist of space (not spacetime) translations $P_{i}$, spacetime dilation $D$, rotations $J_{i j}$ and boosts $W_{i}$.

Algebra

$$
\begin{gathered}
P_{i} \text { and } J_{i j} \text { are those of } \operatorname{ISO}(d), \\
W_{i} \text { is covariant under } J_{i j}, \\
{\left[P_{i}, D\right]=P_{i}} \\
{\left[P_{i}, W_{j}\right]=J_{i j}-\delta_{i j} D .}
\end{gathered}
$$

\section{Matrices}

As the de Sitter group is $\mathrm{SO}(1, \mathrm{D})$, one can use the usual matrix representations for Lorentz generators $M_{\mu v}$, with $\mu=0,1, \ldots, D$.

$$
\begin{aligned}
P_{i} & =M_{0 i}-M_{i D}, \\
D & =M_{0 D}, \\
J_{i j} & =M_{i j}, \\
W_{i} & =M_{0 i} .
\end{aligned}
$$

Levi decomposition

$\mathrm{SO}(1, \mathrm{D})$ is already simple.

\section{A.2 GAUGE GROUPS}

Gauge groups are, roughly speaking, groups of uncountably infinite dimension. Differently from finite groups, they can't be defined abstractly, without reference to any other object. We distinguish two types of gauge groups: 
1. Gauged finite groups, which are the result of taking a prototype abstract group and fibering it over a base spacetime. We can represent them by the prototype group.

2. Diffeomorphism groups, which act directly on the base spacetime. They must be represented by the corresponding spacetime transformations. ${ }^{2}$

Only the diffeomorphism groups need to be listed; for gauged finite groups, we simply classify them by the corresponding abstract group. Neither Ado's theorem nor the Levi decomposition theorem apply to diffeomorphism groups.

\section{A.2.1 Full diffeomorphism group}

The full diffeomorphism group $\operatorname{Diff}(\mathrm{D})$ is the group of complete coordinate covariance:

$$
x^{\mu} \rightarrow f^{\mu}(x),
$$

where $f^{\mu}$ are smooth invertible functions. The group product is function composition.

Diffeomorphisms are generated by the Lie derivative $L_{\xi}$, where $\xi^{\mu}$ is a spacetime vector field.

Algebra

$$
\begin{aligned}
{\left[\mathrm{L}_{\xi_{1}}, \mathrm{~L}_{\xi_{2}}\right] } & =\mathrm{L}_{\xi_{3}} \\
\xi_{3}^{\mu} & =\xi_{1}^{v} \partial_{\nu} \xi_{2}^{\mu}-\xi_{2}^{v} \partial_{\nu} \xi_{1}^{\mu} .
\end{aligned}
$$

\section{A.2.2 Cosmology diffeomorphism group}

The cosmology diffeomorphism group $\operatorname{CDiff}(\mathrm{d})$ is the group of spatial transformations with absolute time:

2 Some physicists would not use the term "gauge group" for diffeomorphism groups; others would be even stricter and reserve the term only for gauged finite groups that are internal and compact (i. e., Yang-Mills). 


$$
\begin{aligned}
t & \rightarrow t, \\
\vec{x} & \rightarrow \vec{f}(t, \vec{x}) .
\end{aligned}
$$

where $\vec{f}$ are smooth invertible functions. The group product is function composition.

Diffeomorphisms are generated by the $L_{\xi}$, where $\xi^{i}$ is a spatial vector field with spacetime dependence.

Algebra

$$
\begin{aligned}
{\left[\mathrm{L}_{\xi_{1}}, \mathrm{~L}_{\xi_{2}}\right] } & =\mathrm{L}_{\xi_{3}} \\
\xi_{3}^{i} & =\xi_{1}^{j} \partial_{j} \xi_{2}^{i}-\xi_{2}^{j} \partial_{j} \xi_{1}^{i} .
\end{aligned}
$$



In Section 7.4.1, we mentioned the isometries of de Sitter spacetime in $\mathrm{D}$ dimenions. Here we provide their full expression in flat slicing, conformal time coordinates.

Under space translations $d^{i}$, dilation $\Lambda$ and rotations $\theta_{i j}$, the coordinates $x^{\mu}=\left(\tau, \chi^{i}\right)$ transform as:

$$
\begin{aligned}
\tau & \rightarrow \wedge \tau, \\
\chi^{i} & \rightarrow \wedge R(\theta)_{j}^{i}\left(x^{j}+d^{j}\right) .
\end{aligned}
$$

where $R(\theta)_{j}^{i}$ is a rotation matrix. More complicated are the dS boosts. We decompose the spatial position vector $\vec{x}$ into one parallel to the direction of the boost, $\vec{x}_{\|}$, and one orthogonal, $\vec{x}_{\perp}$. Then a boost with rapidity $-\infty<\beta<\infty$ along the direction $\vec{u}$ is:

$$
\begin{gathered}
\tau \rightarrow \frac{2 \tau}{\left(1-\mathrm{H}^{2} \chi^{2}\right)+\left(1+\mathrm{H}^{2} \chi^{2}\right) \cosh (\beta)+2 \mathrm{H}\left(\overrightarrow{\mathrm{u}} \cdot \vec{x}_{\|}\right) \sinh (\beta)^{\prime}} \\
\vec{x}_{\|} \rightarrow \frac{2 \vec{x}_{\|} \cosh (\beta)+\overrightarrow{\mathrm{u}} \sinh (\beta)\left(1+\mathrm{H}^{2} \chi^{2}\right) / \mathrm{H}}{\left(1-\mathrm{H}^{2} \chi^{2}\right)+\left(1+\mathrm{H}^{2} \chi^{2}\right) \cosh (\beta)+2 \mathrm{H}\left(\vec{u}^{\prime} \cdot \vec{x}_{\|}\right) \sinh (\beta)^{\prime}} \\
\vec{x}_{\perp} \rightarrow \frac{2 \vec{x}_{\perp}}{\left(1-\mathrm{H}^{2} \chi^{2}\right)+\left(1+\mathrm{H}^{2} \chi^{2}\right) \cosh (\beta)+2 \mathrm{H}\left(\overrightarrow{\mathrm{u}} \cdot \vec{x}_{\|}\right) \sinh (\beta)}
\end{gathered}
$$

where $x^{2} \stackrel{\text { def }}{=}-\tau^{2}+|\vec{x}|^{2}$. 
Upon switching to physical time, $\tau=-e^{-H t}$, and taking the flat spacetime limit $\mathrm{H} \rightarrow 0$, the boost reduces to the usual Lorentz one:

$$
\begin{aligned}
\tau & \rightarrow \cosh (\beta) t-\sinh (\beta)\left(\vec{u} \cdot \vec{x}_{\|}\right) \\
\vec{x}_{\|} & \rightarrow \cosh (\beta) \vec{x}_{\|}-\sinh (\beta) t \vec{u}, \\
\vec{x}_{\perp} & \rightarrow \vec{x}_{\perp}
\end{aligned}
$$




\section{ACKNOWLEDGMENTS}

First, I'd like to thank my mother Lizana and sister Natália for their support during this PhD program.

I am also grateful to my supervisor, Enrico, for the guidance through this research and feedback in writing this thesis, Stefan for being my formal promotor, and Govert for the Dutch translation of the summary.

I acknowledge the Netherlands Organisation for Scientific Research (NWO), funded by the Dutch Ministry of Education, Culture and Science (OCW), for funding my PhD position at Utrecht University. 

Symmetry has always been at the heart of physical theories describing nature, perfectly captured by Noether's theorem. Yet symmetries are arguably at their most interesting when they are spontaneously broken by nature itself. In this thesis, we looked at the structure and consequences of broken spacetime symmetries, meaning that they involve the transformation of spacetime coordinates. Because spacetime and gravity are interlinked, such symmetry breaking patterns often give birth to effective theories of gravitational systems.

Remarkably, in the case of broken spacetime symmetries, famous results encoded by Goldstone's theorem no longer apply. That is to say, there isn't necessarily one bosonic particle for each broken symmetry, and the particles that do exist need not be massless.

To better understand the structure of broken spacetime symmetries, we study methods for constructing effective field theories out of the symmetry breaking pattern. In particular, we present a novel protocol to do this which is better suited for broken diffeomorphisms, which are the symmetries associated with changes in coordinates. Such tools prove useful when studying the effective theory of cosmology, as the expansion of the universe over time breaks changes in the time coordinate.

Indeed, the role of broken symmetries in the effective theory of cosmology is rich. Frequently, to address several issues with the inflationary paradigm (a supposed period of accelerated expansion in the very early universe), a scalar shift symmetry is invoked, which is a kind of internal (not spacetime) symmetry. We study the interplay between the broken time diffeomorphism and this shift, allowing us to constrain the free parameters of the effective theory of cosmology and derive observational checks the theory must satisfy, the so-called soft theorems.

Finally, we also look at the failure of some of the Goldstone particles to exist. By looking at the group-theoretical structure of the symmetries and by examining various examples in flat Minkowski 
spacetime, we are able to better understand why this happens. Armed with this knowledge, we proceed to curved de Sitter spacetime and manage at producing an effective theory with both Goldstone scalars and vectors, a novel result. 


\section{SAMENVATTING}

Symmetrie heeft altijd in het midden van fysische theorieën gestaan die de natuur beschrijven, perfect gevangen door de stelling van Noether. Echter zijn symmetrieën het interessantst wanneer deze spontaan gebroken worden door de natuur zelf. In deze thesis hebben we gekeken naar de structuur en consequenties van gebroken ruimtetijdssymmetrieën, wat betekent dat ze betrekking hebben op de transformatie van ruimtetijdcoördinaten. Omdat de ruimtetijd en zwaartekracht verbonden zijn, leiden zulke symmetriebrekingspatronen vaak tot effectieve theorieën van gravitationele systemen.

Opmerkelijkerwijs gelden beroemde resultaten uit de stelling van Goldstone niet langer in het geval van gebroken ruimtetijdssymmetrieën. Met andere woorden, er is niet noodzakelijkerwijs één bosonisch deeltje voor elke gebroken symmetrie, en de deeltjes die wel bestaan hoeven niet massaloos te zijn.

Om de structuur van gebroken ruimtetijdssymmetrieën beter te begrijpen, bestuderen we methoden om effectieve veldentheorieën te construeren uit het symmetriebrekingspatroon. In het bijzonder presenteren we een nieuw protocol om dit te doen wat beter werkt voor gebroken diffeomorfismes, wat symmetrieën zijn die geassocieerd zijn met veranderingen in coördinaten. Zulk gereedschap blijkt handig voor het bestuderen van de effectieve theorie van kosmologie, aangezien de expansie van het universum over tijd veranderingen in de tijdscoördinaat breekt.

Inderdaad is de rol van gebroken symmetrieën in de effectieve theorie van kosmologie groot. Vaak wordt, om verscheidene moeilijkheden met het inflationaire paradigma (een veronderstelde periode van versnelde expansie in het zeer vroege universum) te addresseren, een beroep gedaan op een scalaire verschuivingssymmetrie, wat een soort van interne (geen ruimtetijd) symmetrie is. We bestuderen de wisselwerking tussen de gebroken tijdsdiffeomorfismes en deze verschuiving, wat ons in staat stelt om de vrije parameters van de effectieve theorie van de kosmologie te beperken, 
en observationele controles af te leiden waar de theorie aan moet voldoen, de zogenaamde zachte stellingen.

Tenslotte kijken we ook naar het niet bestaan van sommige van de Goldstonedeeltjes. Door te kijken naar de groepentheoretische structuur van de symmetrieën en door verscheidene voorbeelden in vlakke Minkowskiruimtetijd te analyseren, zijn we in staat om beter te begrijpen waarom dit gebeurt. Met deze kennis gaan we door naar gekromde de Sitter ruimtetijd en produceren we een effectieve theorie met zowel Goldstonescalairen en vectoren, een nieuw resultaat. 
[1] Rui Shi and Hajime Tanaka. "Impact of local symmetry breaking on the physical properties of tetrahedral liquids." en. In: Proceedings of the National Academy of Sciences 115.9 (Feb. 2018). Publisher: National Academy of Sciences Section: Physical Sciences, pp. 1980-1985. ISSN: 0027-8424, 1091-6490. DOI: 10.1073/pnas. 1717233115.

[2] F. Englert and R. Brout. "Broken Symmetry and the Mass of Gauge Vector Mesons." In: \prl 13.9 (Aug. 1964), pp. 321-323. DoI: 10.1103/PhysRevLett. 13.321.

[3] Peter W. Higgs. "Broken Symmetries and the Masses of Gauge Bosons." In: \prl 13.16 (Oct. 1964), pp. 508-509. Dor: 10.1103/PhysRevLett. 13.508.

[4] Clifford Cheung, Paolo Creminelli, A. Liam Fitzpatrick, Jared Kaplan, and Leonardo Senatore. "The Effective Field Theory of Inflation." English. In: Journal of High Energy Physics 2008.03 (Mar. 2008). arXiv: 0709.0293, pp. 014-014. ISSN: 1029-8479. DOI: $10.1088 / 1126-6708 / 2008 / 03 / 014$.

[5] J James Frost, Kenneth J Pienta, and Donald S Coffey. "Symmetry and symmetry breaking in cancer: a foundational approach to the cancer problem." In: Oncotarget 9.14 (Dec. 2017), pp. 11429-11440. ISSN: 1949-2553. DOI: 10.18632/oncotarget. 22939.

[6] Masaki Miya, Theodore W. Pietsch, James W. Orr, Rachel J. Arnold, Takashi P. Satoh, Andrew M. Shedlock, HsuanChing Ho, Mitsuomi Shimazaki, Mamoru Yabe, and Mutsumi Nishida. "Evolutionary history of anglerfishes (Teleostei: Lophiiformes): a mitogenomic perspective." In: BMC Evolutionary Biology 10.1 (Feb. 2010), p. 58. IsSN: 1471-2148. DOI: 10.1186/1471-2148-10-58. 
[7] Yoichiro Nambu and G. Jona-Lasinio. “Dynamical Model of Elementary Particles Based on an Analogy with Superconductivity. 1." In: Phys. $\backslash$ Rev. 122 (1961), pp. 345-358. DoI: 10.1103/PhysRev. 122.345.

[8] Heinrich Leutwyler. "Phonons as Goldstone Bosons." In: Helv. Phys. Acta 70 (1996).

[9] S. Coleman, J. Wess, and Bruno Zumino. "Structure of Phenomenological Lagrangians. I." In: Physical Review 177.5 (Jan. 1969), pp. 2239-2247. DOI: 10.1103/PhysRev. 177.2239.

[10] Curtis G. Callan, Sidney Coleman, J. Wess, and Bruno Zumino. "Structure of Phenomenological Lagrangians. II." In: Physical Review 177.5 (Jan. 1969), pp. 2247-2250. DOI: 10.1103/ PhysRev. 177.2247.

[11] Gabriele Franciolini, Lam Hui, Riccardo Penco, Luca Santoni, and Enrico Trincherini. "Effective Field Theory of Black Hole Quasinormal Modes in Scalar-Tensor Theories." In: Journal of High Energy Physics 2019.2 (Feb. 2019). arXiv: 1810.07706, p. 127. ISSN: 1029-8479. DOI: 10. 1007/JHEPO2 (2019) 127.

[12] Garrett Goon, Kurt Hinterbichler, and Mark Trodden. "General Embedded Brane Effective Field Theories." In: Physical Review Letters 106.23 (June 2011). arXiv: 1103.6029, p. 231102. ISSN: 0031-9007, 1079-7114. DOI: 10. 1103/PhysRevLett. 106. 231102.

[13] Dmitri Volkov. "Phenomenological Lagrangians." In: Fiz. Elem. Chast. Atom. Yadra 4 3-41 (1973).

[14] E. A. Ivanov and V. I. Ogievetskii. "Inverse Higgs effect in nonlinear realizations." en. In: Theoretical and Mathematical Physics 25.2 (Nov. 1975), pp. 1050-1059. ISSN: 1573-9333. DOI: 10.1007/BF01028947.

[15] I. N. McArthur. "Nonlinear realizations of symmetries and unphysical Goldstone bosons." In: Journal of High Energy Physics 2010.11 (Nov. 2010). arXiv: 1009.3696. ISSN: 1029-8479. DOI: $10.1007 /$ JHEP11 (2010) 140.

[16] Scott Dodelson. Modern Cosmology. en. Google-Books-ID: 3oPRxdXJexcC. Academic Press, Mar. 2003. IsBN: 978-0-12-219141-1. 
[17] Somnath Bharadwaj, Suketu Bhavsar, and Jatush V. Sheth. "The Size of the Longest Filaments in the Universe." In: The Astrophysical Journal 606.1 (May 2004). arXiv: astro-ph/0311342, pp. 25-31. ISSN: 0004-637X, 1538-4357. DOI: 10.1086/382140.

[18] E. L. Wright. "Theoretical Overview of Cosmic Microwave Background Anisotropy." In: arXiv:astro-ph/0305591 (May 2003). arXiv: astro-ph/0305591.

[19] P. A. R. Ade and others. "Planck 2015 results. XVII. Constraints on primordial non-Gaussianity." In: Astron. Astrophys. 594 (2016). _eprint: 1502.01592, A17. DOI: 10 . 1051 / 0004 $6361 / 201525836$.

[20] Wayne Hu and Martin White. "Acoustic Signatures in the Cosmic Microwave Background." In: The Astrophysical Journal 471.1 (Nov. 1996). arXiv: astro-ph/9602019, pp. 30-51. IssN: 0004-637X, 1538-4357. DOI: 10.1086/177951.

[21] Shinji Tsujikawa. "Introductory review of cosmic inflation." In: arXiv:hep-ph/0304257 (Apr. 2003). arXiv: hep-ph/0304257.

[22] H. Bacry and J. Nuyts. "Classification of ten-dimensional kinematical groups with space isotropy." In: Journal of Mathematical Physics 27.10 (Oct. 1986). Publisher: American Institute of Physics, pp. 2455-2457. IssN: 0022-2488. DOI: 10 . 1063/1. 527306.

[23] José Figueroa-O'Farrill. "Classification of kinematical Lie algebras." In: arXiv:1711.05676 [hep-th] (Mar. 2018). arXiv: 1711.05676 .

[24] L. O'Raifeartaigh. "Lorentz Invariance and Internal Symmetry." In: Physical Review 139.4B (Aug. 1965), B1052-B1062. DOI: 10.1103/PhysRev.139.B1052.

[25] Katsumi Yagi. "Super Lie Groups." In: Progress in Differential Geometry. Tokyo, Japan: Mathematical Society of Japan, 1993, pp. 407-412. DOI: 10.2969/aspm/02210407.

[26] S. Ferrara, J. Wess, and B. Zumino. "Supergauge Multiplets and Superfields." In: Phys. Lett. 51B (1974), p. 239. DoI: 10. 1016/0370-2693(74) 90283-4. 
[27] Abdus Salam and J. A. Strathdee. "Supergauge Transformations." In: Nucl. Phys. B76 (1974), pp. 477-482. DoI: 10.1016/ 0550- 3213 (74) 90537 - 9.

[28] Andras Laszlo. "Unification mechanism for gauge and spacetime symmetries." In: Journal of Physics A: Mathematical and Theoretical 50.11 (Mar. 2017). arXiv: 1512.03328, p. 115401. ISSN: 1751-8113, 1751-8121. DOI: 10. 1088/1751-8121/aa5c04.

[29] E. Noether. "Invariante Variationsprobleme." deu. In: Nachrichten von der Gesellschaft der Wissenschaften zu Göttingen, MathematischPhysikalische Klasse 1918 (1918), pp. 235-257.

[30] G. S. Guralnik, C. R. Hagen, and T. W. Kibble. "Global Conservation Laws and Massless Particles." In: \prl 13.20 (Nov. 1964), pp. 585-587. DoI: 10.1103/PhysRevLett.13.585.

[31] Alberto Nicolis and Federico Piazza. "Spontaneous Symmetry Probing." English. In: Journal of High Energy Physics 2012.6 (June 2012). arXiv: 1112.5174. ISSN: 1029-8479. DOI: 10.1007/ JHEPO6 (2012) 025.

[32] E. Fabri and L. E. Picasso. "Quantum Field Theory and Approximate Symmetries." In: Phys. Rev. Lett. 16.10 (Mar. 1966). Publisher: American Physical Society, pp. 408-410. DoI: 10.1103/PhysRevLett. 16.408.2.

[33] Garrett Goon, Kurt Hinterbichler, and Mark Trodden. "Symmetries for Galileons and DBI scalars on curved space." In: Journal of Cosmology and Astroparticle Physics 2011.07 (July 2011). arXiv: 1103.5745, pp. O17-017. ISSN: 1475-7516. DOI: 10.1088/1475-7516/2011/07/017.

[34] Mark Fels and Peter J. Olver. “Moving Coframes: I. A Practical Algorithm." en. In: Acta Applicandae Mathematica 51.2 (Apr. 1998), pp. 161-213. IsSN: 0167-8019, 1572-9036. DOI: 10. 1023/A: 1005878210297.

[35] Mark Fels and Peter J. Olver. "Moving Coframes: II. Regularization and Theoretical Foundations." en. In: Acta Applicandae Mathematica 55.2 (Jan. 1999), pp. 127-208. ISSN: 0167-8019, 1572-9036. DOI: 10. 1023/A : 1006195823000. 
[36] C. P. Burgess. "Goldstone and Pseudo-Goldstone Bosons in Nuclear, Particle and Condensed-Matter Physics." In: Physics Reports 330.4 (June 2000). arXiv: hep-th/9808176, pp. 193-261. ISSN: 03701573. DOI: 10.1016/S0370-1573 (99)00111-8.

[37] Garrett Goon, Kurt Hinterbichler, Austin Joyce, and Mark Trodden. "Galileons as Wess-Zumino Terms." In: Journal of High Energy Physics 2012.6 (June 2012). arXiv: 1203.3191, p. 4. ISSN: 1029-8479. DOI: 10.1007/JHEPO6 ( 2012 ) 004.

[38] Peter J. Olver, Juha Pohjanpelto, and Francis Valiquette. “On the Structure of Lie Pseudo-Groups." In: Symmetry, Integrability and Geometry: Methods and Applications (July 2009). arXiv: 0907.4086 version: 1. ISSN: 18150659. DOI: 10. 3842 / SIGMA . 2009.077.

[39] Grigorii B. Pivovarov and Victor T. Kim. "On Naturalness of Scalar Fields and Standard Model." In: Physical Review D 78.1 (July 2008). arXiv: 0712.0402, p. O16001. ISSN: 1550-7998, 1550-2368. DoI: 10.1103/PhysRevD.78.016001.

[40] Daniel Baumann and Liam McAllister. Inflation and String Theory. _eprint: 1404.2601. Cambridge University Press, 2015. ISBN: 978-1-107-08969-3 978-1-316-23718-2.

[41] Antonio Padilla. "Lectures on the Cosmological Constant Problem." In: arXiv:1502.05296 [astro-ph, physics:gr-qc, physics:hepph, physics:hep-th] (Feb. 2015). arXiv: 1502.05296.

[42] Nima Arkani-Hamed, Lubos Motl, Alberto Nicolis, and Cumrun Vafa. "The String landscape, black holes and gravity as the weakest force." In: JHEP o706 (2007). _eprint: hepth/o601001, p. o6o. DoI: 10.1088/1126-6708/2007/06/060.

[43] Renata Kallosh, Andrei D. Linde, Dmitri A. Linde, and Leonard Susskind. "Gravity and global symmetries." In: Phys. Rev. D52 (1995)._eprint: hep-th/9502069, pp. 912-935. DOI: 10.1103/ PhysRevD.52.912.

[44] Tom Banks and Nathan Seiberg. "Symmetries and Strings in Field Theory and Gravity." In: Phys. Rev. D83 (2011)._eprint: 1011.5120, p. 084019. DOI: 10.1103/PhysRevD.83.084019. 
[45] Lasha Berezhiani and Justin Khoury. "Theory of dark matter superfluidity." In: Phys. Rev. D92 (2015)._eprint: 1507.01019, p. 103510. DOI: 10.1103/PhysRevD.92.103510.

[46] Marco Celoria, Denis Comelli, and Luigi Pilo. "Fluids, Superfluids and Supersolids: Dynamics and Cosmology of Self Gravitating Media." In: JCAP 1709.09 (2017)._eprint: 1704.00322, p. 036. DOI: 10.1088/1475-7516/2017/09/036.

[47] Marco Celoria, Denis Comelli, and Luigi Pilo. "Intrinsic Entropy Perturbations from the Dark Sector." In: (2017). _eprint: 1711.01961.

[48] Alberto Nicolis, Riccardo Penco, Federico Piazza, and Rachel A. Rosen. "More on gapped Goldstones at finite density: More gapped Goldstones." In: JHEP 1311 (2013). _eprint: 1306.1240, p. 055. DOI: 10. 1007/JHEP11 (2013) 055.

[49] Alberto Nicolis, Riccardo Penco, and Rachel A. Rosen. "Relativistic Fluids, Superfluids, Solids and Supersolids from a Coset Construction." In: Physical Review D 89.4 (Feb. 2014). arXiv: 1307.0517, p. 045002. IsSN: 1550-7998, 1550-2368. DOI: 10.1103/PhysRevD . 89.045002.

[50] Alberto Nicolis, Riccardo Penco, Federico Piazza, and Riccardo Rattazzi. "Zoology of condensed matter: Framids, ordinary stuff, extra-ordinary stuff." In: [cond-mat, gr-qc, hep-th] (Jan. 2015). arXiv: 1501.03845.

[51] Alberto Nicolis and Federico Piazza. "A relativistic nonrelativistic Goldstone theorem: gapped Goldstones at finite charge density." In: Physical Review Letters 110.1 (Jan. 2013). arXiv: 1204.1570, p. O11602. ISSN: 0031-9007, 1079-7114. DOI: 10.1103/PhysRevLett. 110.011602.

[52] Steven Weinberg. "Adiabatic modes in cosmology." In: Phys. Rev. D67 (2003). _eprint: astro-ph/o302326, p. 123504. DOI: 10.1103/PhysRevD . 67. 123504.

[53] Kurt Hinterbichler, Lam Hui, and Justin Khoury. "An Infinite Set of Ward Identities for Adiabatic Modes in Cosmology." In: JCAP 1401 (2014)._eprint: 1304.5527, p. 039. DOI: 10.1088/ $1475-7516 / 2014 / 01 / 039$. 
[54] Valentin Assassi, Daniel Baumann, and Daniel Green. "Symmetries and Loops in Inflation." In: JHEP 02 (2013). _eprint: 1210.7792, p. 151. DOI: 10.1007/JHEPO2 (2013) 151.

[55] A. Kehagias and A. Riotto. "Operator Product Expansion of Inflationary Correlators and Conformal Symmetry of de Sitter." In: Nuclear Physics B 864.3 (Nov. 2012). arXiv: 1205.1523, pp. 492-529. ISSN: 05503213. DOI: 10.1016/j . nucl physb. 2012. 07.004.

[56] Paolo Creminelli, Jorge Norena, and Marko Simonovic. "Conformal consistency relations for single-field inflation." In: JCAP 1207 (2012)._eprint: 1203.4595, p. 052. DOI: 10.1088/ $1475-7516 / 2012 / 07 / 052$.

[57] Steven Weinberg. The Quantum Theory of Fields. en. ISBN: 9780521670531 9780521550017 9781139644167 Library Catalog: www.cambridge.org Publisher: Cambridge University Press. June 1995. Dor: 10.1017/CB09781139644167.

[58] William H. Kinney. "Horizon crossing and inflation with large eta." In: Phys. Rev. D72 (2005)._eprint: gr-qc/0503017, p. 023515. DOI: 10.1103/PhysRevD.72.023515.

[59] Nima Arkani-Hamed, Paolo Creminelli, Shinji Mukohyama, and Matias Zaldarriaga. "Ghost Inflation." English. In: Journal of Cosmology and Astroparticle Physics 2004.04 (Apr. 2004). arXiv: hep-th/o312100, pp. o01-001. ISSN: 1475-7516. DOI: 10. 1088/1475- 7516/2004/04/001.

[6o] Mohammad Hossein Namjoo, Hassan Firouzjahi, and Misao Sasaki. "Violation of non-Gaussianity consistency relation in a single field inflationary model." In: Europhys. Lett. 101 (2013)._eprint: 1210.3692, p. 39001. DOI: 10.1209/0295-5075/ $101 / 39001$.

[61] Leonardo Senatore, Kendrick M. Smith, and Matias Zaldarriaga. "Non-Gaussianities in Single Field Inflation and their Optimal Limits from the WMAP 5-year Data." In: JCAP 1001 (2010)._eprint: 0905.3746, p. 028. DOI: 10 . 1088/1475 - 7516/ 2010/01/028. 
[62] Ian Low and Aneesh V. Manohar. "Spontaneously Broken Spacetime Symmetries and Goldstone's Theorem." en. In: Physical Review Letters 88 (2002) (Oct. 2001). arXiv: hep-th/o110285. DOI: 10.1103/PhysRevLett. 88.101602.

[63] Remko Klein, Diederik Roest, and David Stefanyszyn. "Spontaneously Broken Spacetime Symmetries and the Role of Inessential Goldstones." In: Journal of High Energy Physics 2017.10 (Oct. 2017). arXiv: 1709.03525, p. 51. ISSN: 1029-8479. DOI: $10.1007 /$ JHEP10 ( 2017 ) 051.

[64] Tomáš Brauner and Haruki Watanabe. "Spontaneous breaking of spacetime symmetries and the inverse Higgs effect." In: Physical Review D 89.8 (Apr. 2014). arXiv: 1401.5596, p. 085004. ISSN: 1550-7998, 1550-2368. DOI: 10.1103 / PhysRevD . 89 . 085004.

[65] Remko Klein, Emanuel Malek, Diederik Roest, and David Stefanyszyn. "A No-go Theorem for a Gauge Vector as a Space-time Goldstone." In: Physical Review D 98.6 (Sept. 2018). arXiv: 1806.06862, p. 065001. ISSN: 2470-0010, 2470-0029. DOI: 10.1103/PhysRevD. 98.065001.

[66] Kurt Hinterbichler, Austin Joyce, and Justin Khoury. “Nonlinear Realizations of Conformal Symmetry and Effective Field Theory for the Pseudo-Conformal Universe." In: Journal of Cosmology and Astroparticle Physics 2012.06 (June 2012). arXiv: 1202.6056, pp. 043-043. ISSN: 1475-7516. DOI: 10.1088/ $1475-7516 / 2012 / 06 / 043$.

[67] Kurt Hinterbichler and Austin Joyce. "A Hidden Symmetry of the Galileon." In: Physical Review D 92.2 (July 2015). arXiv: 1501.07600, p. 023503. ISSN: 1550-7998, 1550-2368. DOI: 10 . 1103/PhysRevD. 92.023503.

[68] Sebastian Garcia-Saenz, Jonghee Kang, and Riccardo Penco. "Gauged Galileons." In: Journal of High Energy Physics 2019.7 (July 2019). arXiv: 1905.05190, p. 81. ISSN: 1029-8479. DOI: 10.1007/JHEP07 (2019) 081. 
[69] Paolo Creminelli, Marco Serone, and Enrico Trincherini. "Nonlinear Representations of the Conformal Group and Mapping of Galileons." In: Journal of High Energy Physics 2013.10 (Oct. 2013). arXiv: 1306.2946, p. 40. ISSN: 1029-8479. DOI: 10.1007/ JHEP10 ( 2013 ) 040.

[70] James Bonifacio, Kurt Hinterbichler, Austin Joyce, and Rachel A. Rosen. "Shift Symmetries in (Anti) de Sitter Space." In: Journal of High Energy Physics 2019.2 (Feb. 2019). arXiv: 1812.08167, p. 178. ISSN: 1029-8479. DOI: 10. 1007/JHEPO2 (2019) 178.

[71] Gianmassimo Tasinato. "Cosmic Acceleration from Abelian Symmetry Breaking." In: Journal of High Energy Physics 2014.4 (Apr. 2014). arXiv: 1402.6450, p. 67. ISSN: 1029-8479. DOI: 10. 1007/JHEP04 ( 2014 ) 067.

[72] Lavinia Heisenberg. "Generalised Proca Theories." In: [hep-th] (May 2017). arXiv: 1705.05387.

[73] Alberto Nicolis, Riccardo Rattazzi, and Enrico Trincherini. "The galileon as a local modification of gravity." In: Physical Review D 79.6 (Mar. 2009). arXiv: 0811.2197, p. 064036. ISSN: 1550-7998, 1550-2368. DoI: 10.1103/PhysRevD.79.064036.

[74] James Bonifacio, Kurt Hinterbichler, Laura A. Johnson, and Austin Joyce. "Shift-Symmetric Spin-1 Theories." In: Journal of High Energy Physics 2019.9 (Sept. 2019). arXiv: 1906.10692, p. 29. ISSN: 1029-8479. DOI: 10 . 1007/JHEPO9 (2019) 029.

[75] E. Inonu and E. P. Wigner. "On the Contraction of Groups and Their Representations." In: Proceedings of the National Academy of Sciences of the United States of America 39.6 (June 1953), pp. 510-524. ISSN: 0027-8424.

[76] Jean-Marc Lévy-Leblond. "Une nouvelle limite non-relativiste du groupe de Poincaré." fra. In: Annales de l'I.H.P. Physique théorique 3.1 (1965). Publisher: Gauthier-Villars, pp. 1-12. ISSN: 0246-0211. 